The Reinhardt Thiessen

Coal Thin-Section Slide Collection of the U.S. Geological SurveyCatalog and Notes

G E O L O G I C A L S U R V E Y B U L L E T I N 1432 


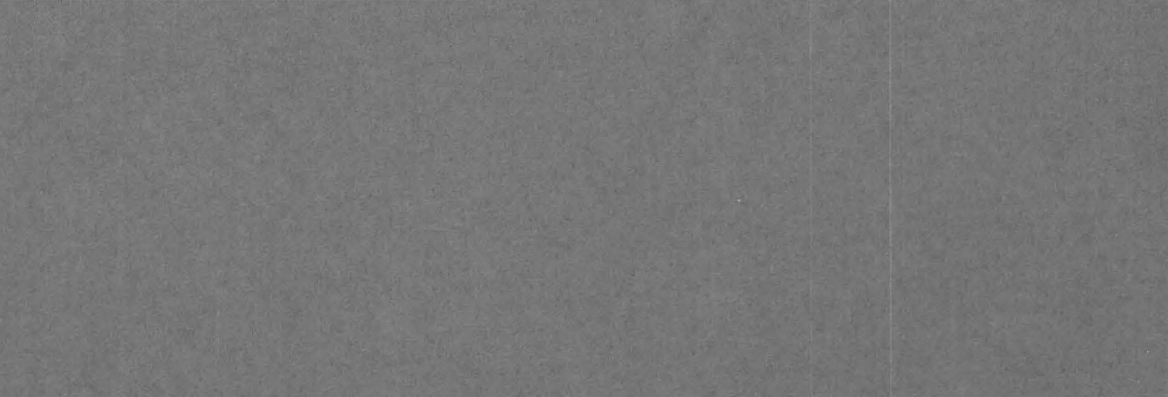

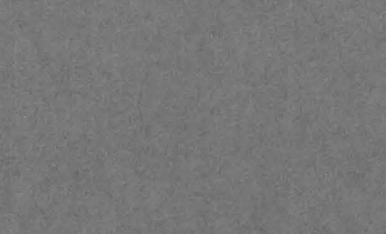

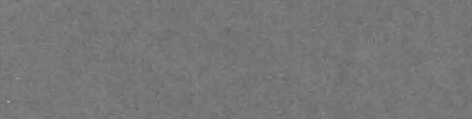

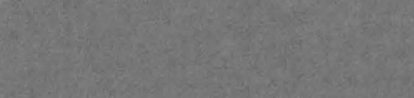

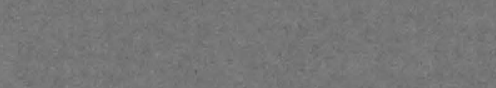

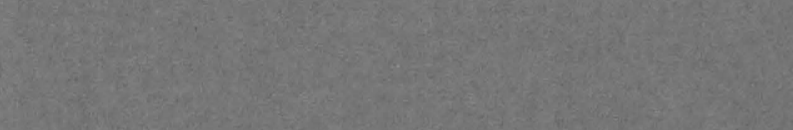

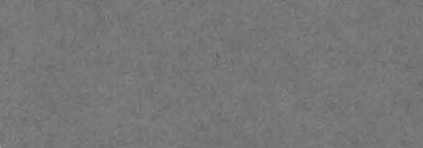

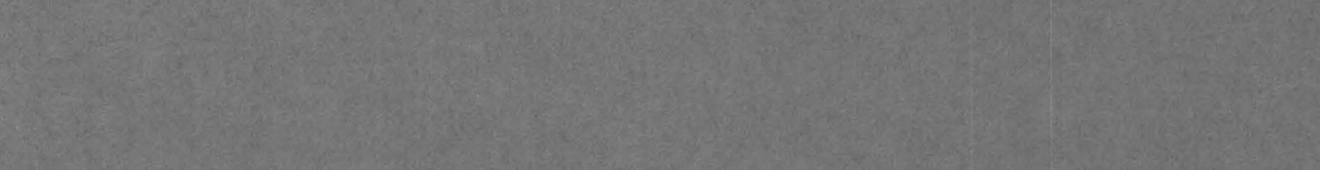

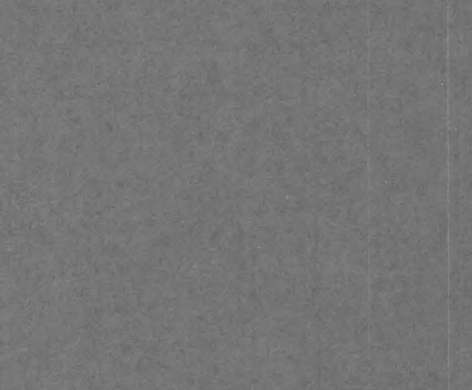

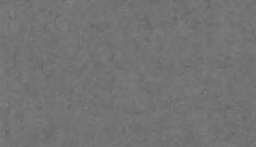
(2)

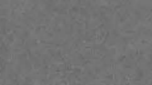

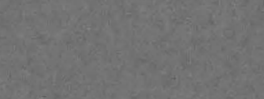

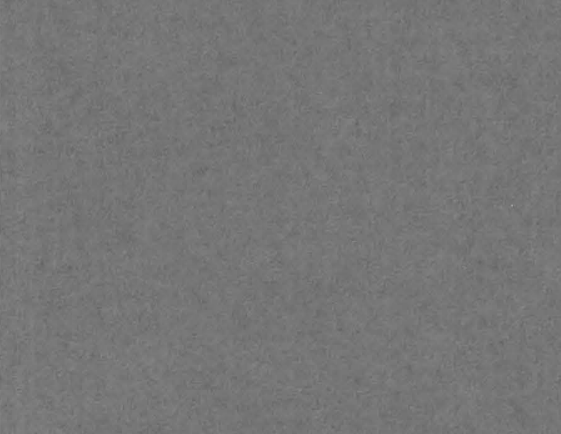
3.t:

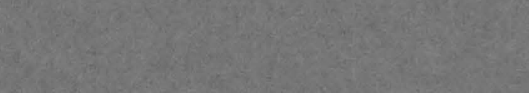

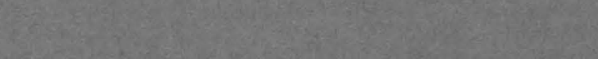

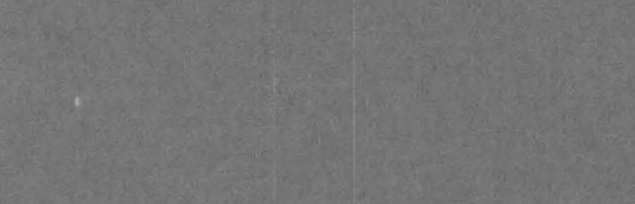

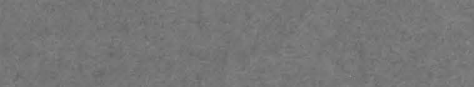

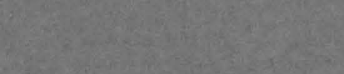
(2)

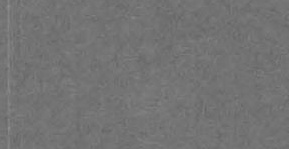

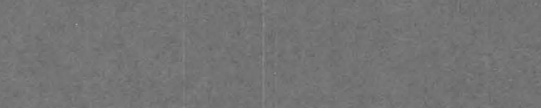

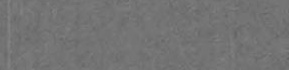
Lit.

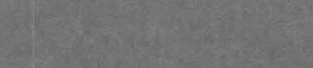

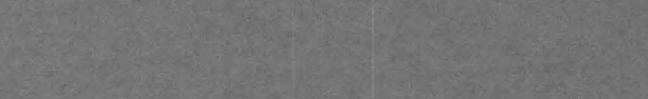

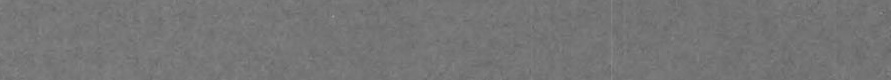

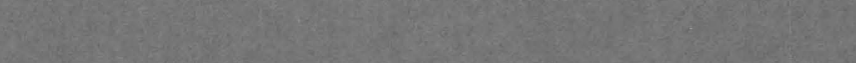

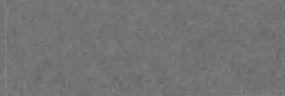




\section{The Reinhardt Thiessen}

\section{Coal Thin-Section Slide Collection}

of the U.S. Geological Survey-

Catalog and Notes

By JAMES M. SCHOPF and ORRIN G. OFTEDAHL

G E O L O G I C A L S U R V E Y B U L L E T I N 14432

$A$ guide to the collection of more than 19,000 slides from 682 localities within and outside the United States, and a bibliography of Reinhardt Thiessen

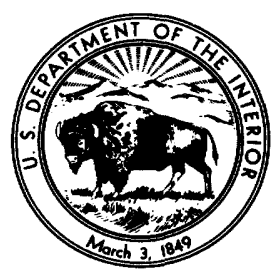




\section{UNITED STATES DEPARTMENT OF THE INTERIOR}

THOMAS S. KLEPPE, Secretary

GEOLOGICAL SURVEY

V. E. McKelvey, Director

Library of Congress Cataloging in Publication Data

Schopf, James Morton, 1911 -

The Reinhardt Thiessen coal thin-section slide collection of the U.S. Geological Survey.

(Geological Survey Bulletin 1432)

Bibliography: p.

Supt. of Docs. no.: I 19.3:1432

1. Coal-Catalogs and collections. 2. Thin sections (Geology)-Catalogs and collections.

3. United States Geological Survey.

I. Oftedahl, Orrin G., joint author. II. Thiessen, Reinhart, 1867-1938. III. Title. IV. Series: United States Geological Survey Bulletin 1432.

QE75.B9 no. 1432 [TN800] 557.3'08s [553'.2] 76-608235

For sale by the Superintendent of Documents, U.S. Government Printing Office

Washington, D.C. 20402

Stock Number 024-001-02921-9 


\section{CONTENTS}

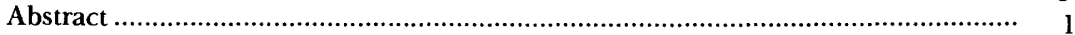

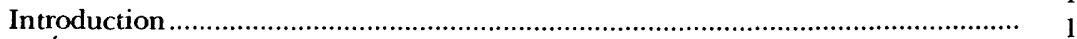

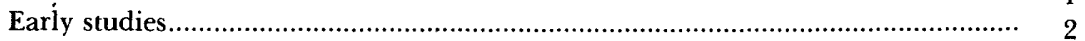

A quantitative system ...................................................................... 4

Organization of the catalog................................................................................... 6

Catalog of the thin-section collection ................................................................ 8

Abbreviations used in catalog listings ......................................................... 8

Areas and subjects preceding the slide box numbers ............................... 8

Miscellaneous abbreviations.................................................................. 9

Unit 1. Appalachian Basin.......................................................................... 9

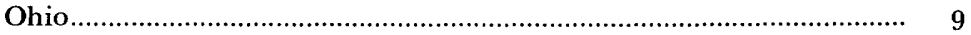

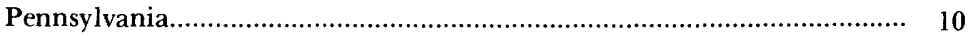

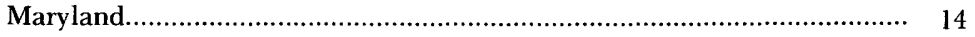

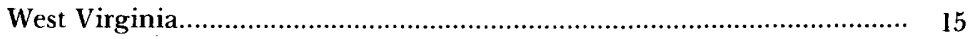

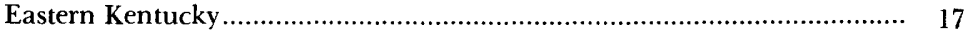

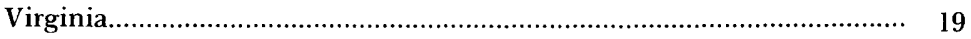

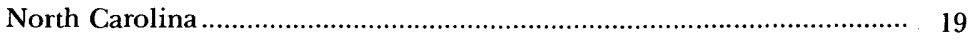

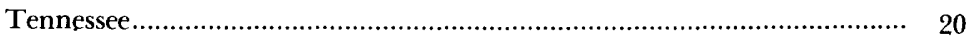

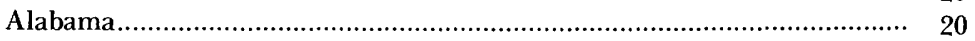

Unit 2. Interior Basin ............................................................................ 21

Western Kentucky ........................................................................... 21

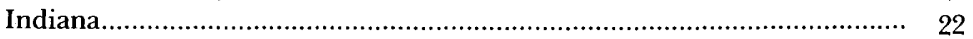

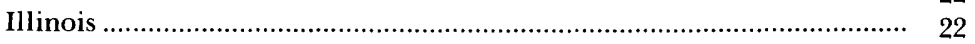

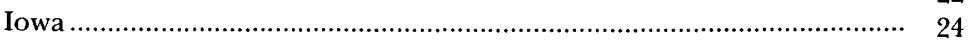

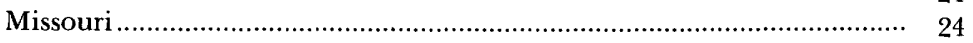

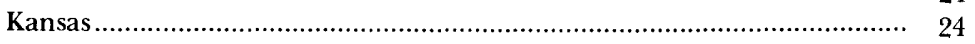

Unit 3. Other Paleozoic basins.................................................................... 24

Arkansas (Paleozoic) ......................................................................... 24

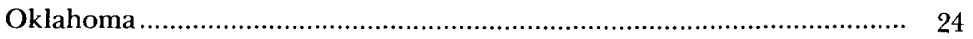

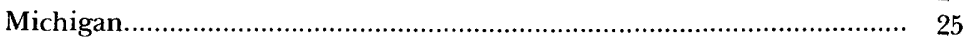

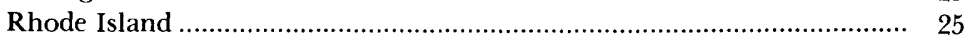

Unit 4. Rocky Mountains and lignite areas ............................................... 25

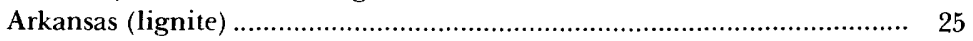

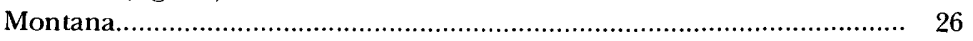

North Dakota........................................................................ 26

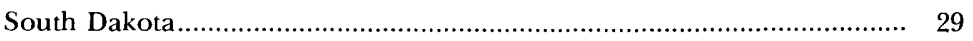

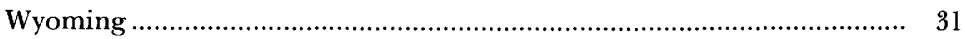

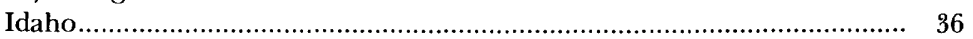

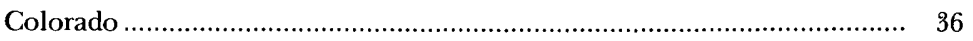

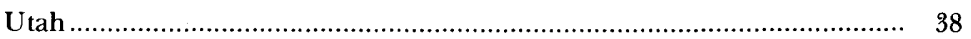

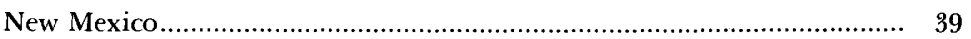

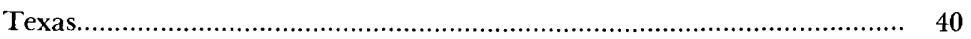

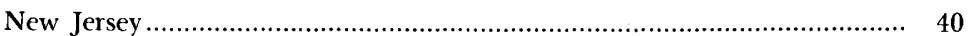


Catalog of the thin-section collection-Continued Page

Unit 5. West coast States and Alaska ........................................................ 40

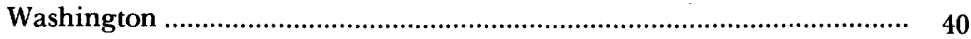

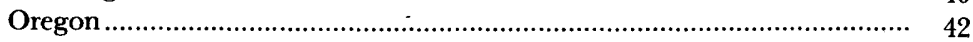

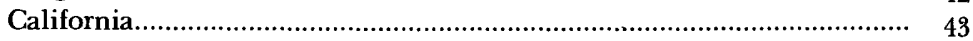

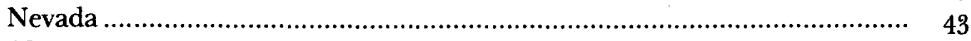

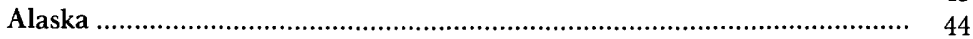

Unit 6. Foreign areas............................................................................... 44

Antarctica.................................................................................... 44

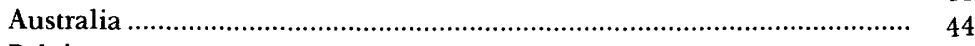

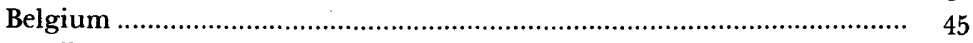

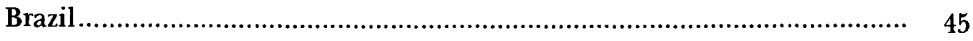

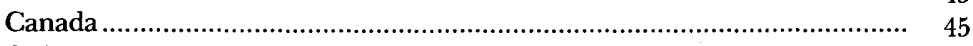

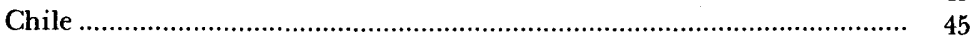

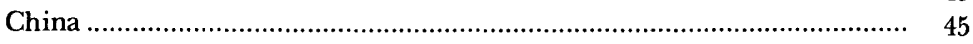

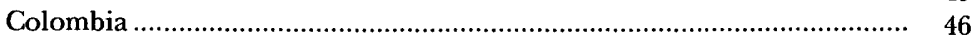

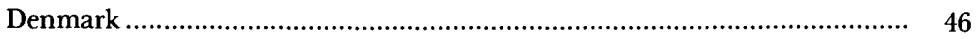

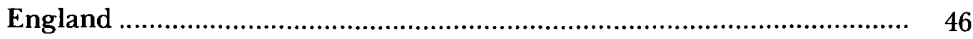

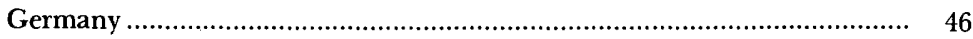

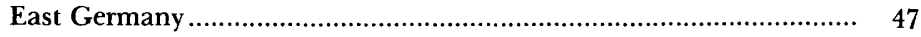

West Germany …............................................................................ 47

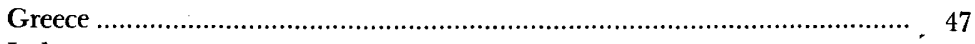

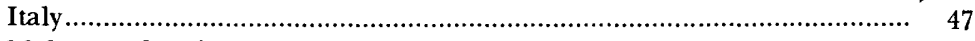

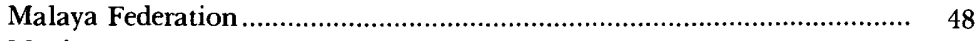

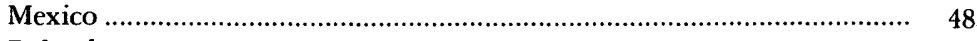

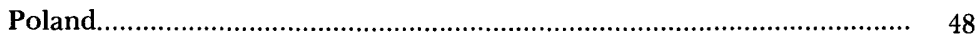

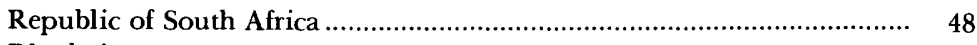

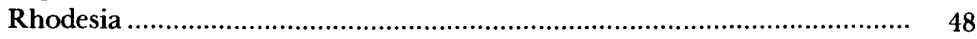

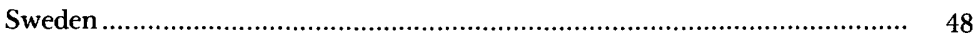

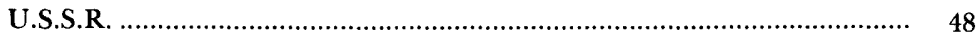

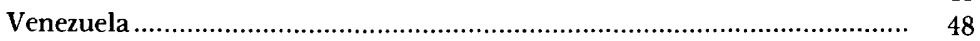

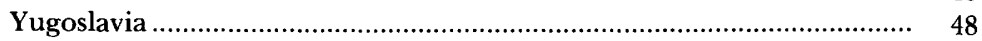

Units 7-9. Special categories...................................................................... 49

7. Lomax, Jeffrey, F. D. R., and Thiessen early studies ……................. 49

Lomax sections......................................................................... 49

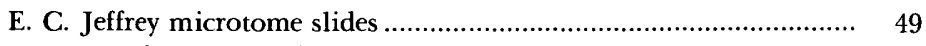

F. D. R. microtome slides ............................................................ 49

R. Thiessen's early studies ........................................................... $\quad 50$

8. Oil shale and related rocks............................................................. 51

United States ....................................................................... 51

Foreign ........................................................................... 52

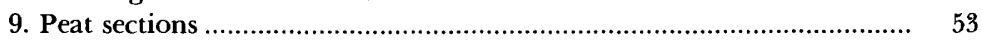

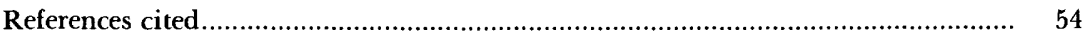

Bibliography of Reinhardt Thiessen.......................................................... 54 


\title{
THE REINHARDT THIESSEN COAL THIN-SECTION SLIDE COLLECTION OF THE U.S. GEOLOGICAL SURVEY- CATALOG AND NOTES
}

\author{
By James M. Schopf and Orrin G. Oftedahl
}

\section{ABSTRACT}

The Reinhardt Thiessen Coal Thin-Section Slide Collection of more than 19,000 slides from 682 different localities includes abundant examples of coal from most American coal fields and from other parts of the world. The collection includes examples used in early studies of coal, on which the first quantitative system of coal petrography was based, and is of historic interest in its relation to studies of the origin of coal and coal classification. All available sections studied at the U.S. Bureau of Mines and at the U.S. Geological Survey, using Thiessen's system of coal petrography, are included, as well as sections of various oil shales and peat.

The catalog is organized according to coal basins and geographic areas, as is the collection, and is intended to facilitate reference. The bibliography includes a complete list of the writings of Reinhardt Thiessen, nearly all of which are pertinent to his coal studies.

\section{INTRODUCTION}

The Reinhardt Thiessen Coal .Thin-Section Slide Collection consists of more than 19,000 thin sections of coal from all the important American coal fields, and many sections from important coal beds in other parts of the world. It includes the basic materials prepared during Thiessen's career with the U.S. Bureau of Mines, to which have been added the similar section preparations made by the U.S. Geological Survey at Columbus, Ohio, during the past 23 years. We are indebted to Mr. J. T. McCartney of the U.S. Bureau of Mines for the transfer to the Geological Survey of much of the original thin-section collection so that all available reference materials can be consolidated and more effectively cared for.

Thiessen devised the first quantitative system of coal petrography, and as the collection now represents nearly all the coal beds studied by this method, it is appropriate that the collection bear his name. The permanent preparations still serve as voucher examples on which published 
analyses are based. This catalog of the collection indicates the materials that are readily available and includes a bibliography of Thiessen's publications. Some of the historical developments that led to the accumulation of this important reference collection are described. Arrangements for use of the collection may be made at the U.S. Geological Survey National Center, Reston, Va.

\section{EARLY STUDIES}

In autumn 1907 Reinhardt Thiessen began work for the U.S. Geological Survey. He was a young Ph. D., fresh from C. J. Chamberlain's laboratory for plant histology at the University of Chicago, where he had completed a dissertation on cycad structure (Thiessen, 1908). He was associated with Survey geologists David White and F. H. Knowlton, stationed at the U.S. National Museum in Washington, D.C., and there he began his career of studies on the constitution of coal. When the Technologic Branch of the Geological Survey became the nucleus of the separate Bureau of Mines in 1910, Thiessen moved with other members of the technological staff to Pittsburgh, Pa., where he continued his studies for the rest of his life.

In 1913 Thiessen contributed a significant and well-illustrated chapter to Bureau of Mines Bulletin 38, the now-classic volume on the origin of coal, other parts being written by David White and C. A. Davis. In that chapter Thiessen gave much attention to technical methods that would prepare coal for microscopic examination without causing more alteration or change than could possibly be avoided. He discussed the Jeffrey method (Jeffrey and Chrysler, 1906; Jeffrey, 1916) of softening and celloidin-embedding for microtome sectioning. The softening procedure used in this method, however, induced changes (artifacts) in the structure of the coal. Also discussed was the Lomax procedure (Lomax, 1911, 1922), in which larger sections were prepared by grinding; this method avoids chemical alteration. Lomax was a renowned British preparator of thin sections of permineralized plant tissues in coal balls; he used much the same method in preparing thin sections of coal. Many of Lomax's sections are still preserved and, from the standpoint of size, are remarkable products of the lapidarist's art. Alteration of inherent structure was avoided in these sections, but few of the large sections could be produced to the critical thinness that Thiessen later demonstrated is essential for resolution of significant microscopic features. All these methods were investigated by Thiessen with the objective of resolving significant microscopic detail and avoiding artifacts that would interfere with observation of structures naturally inherent in the coal.

Thiessen's thin-section procedure was perfected as his studies continued during World War I. These studies resulted in a report (Thiessen, 1920) on structure in Paleozoic bituminous coals, in which Thiessen classified coal into essentially two components- the coarse- 
textured anthraxylon, derived from the woody or sclerotic fragments of plants, and the fine-textured matrix, which he termed "attritus." This work led to the establishment of Thiessen's quantitative method of petrographic analysis, which was first applied in the cooperative Bureau of Mines-American Gas Association program of the 1930's.

Thiessen's characterization of anthraxylon in 1920 set off a controversy with Dr. Marie Stopes, who had been engaged in somewhat similar studies in British laboratories during the war (Stopes and Wheeler, 1918). While Thiessen's report was in press, Stopes (1919) published a somewhat similar terminology for coal ingredients, in which she used the terms "vitrain," "clarain," "durain," and "fusain." She had described these ingredients essentially as megascopic band types, but, relying on the study of Lomax sections for microscopic details, she regarded vitrain as a consolidated colloidal gel lacking any vestige of tissue structure, not as the result of tissue consolidation and alteration, as implied by Thiessen's description of anthraxylon. The question naturally arose as to what extent anthraxylon was equivalent to vitrain.

If the two were equivalent, then the differing descriptions by Stopes and Thiessen raised a serious question as to the origin of this major constituent of coal. That the bright bands in American coal were derived from tissue had been firmly established by Thiessen's studies, but whether the bands in British coal were of similar derivation was not clear. This question was not resolved until 1926, when Thiessen, during a visit to England, studied British coal and demonstrated the ubiquity of tissue structure in vitrain bands that Stopes supposed had a uniform gel like composition. The inherent similarity of anthraxylon and vitrain thus was proved; only the method of quantitative estimation-megascopic or microscopic study-differed. However, this critical difference was obscured because at that time, quantitative coal-petrologic studies were not systematized, and only qualitative, or semiquantitative estimates, at best, were generally reported.

The need for a scientific system of coal classification in this country was recognized by many who had been concerned with coal resources, including members of the Geological Survey and Bureau of Mines. Several systems had been proposed. All had some disadvantages, and none of the systems had been widely adopted or generally used. Coal classification should indicate concisely the general characteristics that are important in practical applications. It should minimize the need for specific analyses, because facilities for these determinations are not always at hand. Classification is intended to group together coals that have similar properties and to distinguish those that have significantly different characteristics. The movement that led to standardization of an appropriate system was instituted by George $\mathbf{H}$. Ashley, at that time Pennsylvania State Geologist, formerly a geologist with the U.S. Geol- 
ogical Survey, who had been detailed for work with the Solid Fuels Administration during World War I. His broad experience emphasized the need for a classification, and the use-classification that he advocated (Ashley, 1920, 1923, 1926) served as an admirable starting point for detailed consideration by all who were concerned with uses and occurrences of coal. Studies of this important problem were initially sponsored by the American Institute of Mining Engineers (AIME) in a series of programs and symposia. Later on, responsibilities were divided for detailed consideration by committees operating under the auspices of the American Society for Testing and Materials (ASTM). Chemical analyses were primarily emphasized, but the extent to which original plant composition influenced the results of chemical analysis remained unresolved. Thiessen clearly discerned the problem and contributed discussions at several symposia. (See bibliography.)

\section{A QUANTITATIVE SYSTEM}

Thiessen's method of quantitative microscopic analysis was devised largely as a means of providing numerical data about the lithologic contribution of various kinds of coalified plant material. His success was due, in part, to the administrative support and urging of Dr. A. C. Fieldner, initially in his capacity as Superintendent of the Pittsburgh Experiment Station and later as Chief of the Fuels and Explosives Branch of the U.S. Bureau of Mines. About 1930 Fieldner assigned Thiessen the principal task of producing a quantitative coal-petrologic system. Thiessen had participated in several symposia that showed the need for a system indicating concisely the inherent plant composition of coal. Fieldner had then taken a most active part in organizing a Committee on Coal and Coke under the ASTM which operated under rules and procedures of the American Standards Association.

In 1931 Thiessen described the general method he used for determining microscopic characteristics and provided the first quantified results for samples from the Taggart coal bed in Wise County, Va. Subsequent microscopic studies were carried out by similar methods and dealt with samples from the Elkhorn coal of Letcher County, Ky., the Chilton bed of Logan County, W. Va., the Black Creek coal bed of Alabama, the Pittsburgh coal bed at several localities in Pennsylvania, the Herrin (No. 6) coal bed of Illinois, and many others, as indicated in the bibliography.

Thiessen did not work entirely by himself. After 1930, he was ably assisted by Hugh J. O'Donnell and George C. Sprunk. O'Donnell developed into a superb thin-section preparator and was largely responsible for preparing consistent series of uniform thin sections through the thickness of a great variety of coal beds, such as the Matanuska coals in Alaska, Arkansas lignite, and Meadow Branch coal in West Virginia. Prior to O'Donnell's work with the Bureau, Thiessen had used the hack- 
saw method of shaping blocks for sectioning, which was advocated by Lomax and which is still in common use for some purposes (Allman and Lawrence, 1972). However, this was the period in which the resinbonded silicon carbide cut-off wheel was being developed, primarily for shaping materials in metallurgy. O'Donnell saw these wheels used in the Metallurgy Section of the Experiment Station at Pittsburgh and was responsible for adapting them for cutting and shaping coal blocks rapidly without shattering, and cleanly without contamination.

George C. Sprunk, trained in college as a chemist, became an expert coal microscopist. Systematic application of the Whipple Disc Counting Technique, which had been introduced by Thiessen, was meticulously carried out by Sprunk for many of the series of coal sections. After Thiessen's death, Sprunk (1942) was responsible for determining that the relatively opaque constituents of coal-fusain and opaque attrituswere much more resistant to liquefaction under test conditions for hydrogenation than were the translucent constituents. Both O'Donnell and Sprunk provided able assistance in preparing the extensive series of partially carbonized test specimens that enabled Thiessen to establish conclusively that the anthraxylous part of the coal forms the "incipient vacuoles" in coking and is responsible for the fusion properties of coals that have coking propensity. O'Donnell, Sprunk, and Thiessen constituted an ideal working research team, which was terminated in 1938 by Thiessen's untimely death. By this time, a systematic procedure had been established requiring oriented specimens. Unfortunately, the system is not suited to bulk-sampling procedures or to study of broken and disoriented specimens. Discussions of the system have been provided by Parks and O'Donnell (1949) and by Schopf (1956). The Thiessen system has been so widely used that it provides the most critical basis for detailed comparison of the in situ petrographic composition of deposits of American coal.

In 1943 Sprunk was called from his work with the Bureau of Mines by more critical work in Detroit, and later by family responsibilities at his parents' home in Adrian, Mich. J. M. Schopf was appointed to take his place at Pittsburgh on studies largely concerned with wartime exploration for western coking coal. Thin sections prepared in connection with most of these investigations are included in the collection and are reported in this catalog. Upon Schopf's transfer to the U.S. Geological Survey to take up paleobotanical studies, B. C. Parks was appointed to aid in coal-petrologic studies with the U.S. Bureau of Mines. One of the notable later achievements of Parks and O'Donnell (1956) was a summary of the results of all coal petrographic studies made at the Bureau of Mines to 1955, by Thiessen's method, on American coal beds.

In 1949, when a search began for uranium in western low-sulfur coal deposits, Schopf was placed in charge of the coal geology laboratory of 
the U.S. Geological Survey in Columbus, Ohio. In addition to the systematic processing of coal cores for chemical determination, an objective of this work was to determine whether any particular plant constituents served as uranium accumulators. Many coals, from northwestern South Dakota, southwestern North Dakota, and from the Red Desert area and the Powder River basin, Wyoming, were investigated, using a more detailed elaboration of the Thiessen method. This laboratory work showed conclusively that no individual plant component was responsible for uranium accumulation in coal; however, a correlation was found between permeability of coal and uranium-bearing ground water. The more permeable fine-textured attrital coal contained far more uranium than denser adjacent woody layers. These results had also been predicted on the basis of field studies. All thin sections of western coal studied for uranium are in the Thiessen Coal Thin-Section Slide Collection.

In addition to his coal studies, Thiessen $(1920,1925,1929,1930,1934$, 1936-37) investigated the composition of peat and of oil shale. Peat was thin sectioned after it had been compressed over a period of weeks or months to remove excess water and to eliminate much of the air. Oil shale, a denser rock, is generally amenable to the coal thin-section method of preparation without special treatment. Sections of carbonaceous rocks, resulting from Thiessen's studies and from other studies at the U.S. Geological Survey coal geology laboratory in Columbus, Ohio, during the past 25 years, also are in the collection. Included are examples of Australian "kerosene shale" and of torbanite from Scotland, from Autun, France, and from Ermelo, South Africa. The compositions of these rocks all contrast strongly with those of samples from the Green River oil shale in the Western United States and from Albertite deposits in New Brunswick, Canada. The Broxburn oil shale, most recently mined in Scotland, contrasts strongly with all of these. The marine Chattanooga and Ohio black shales also are different and are shown to be closely allied with the black "slates" overlying coal beds. No other method of study shows as clearly the differences and similarities of these various kinds of carbonaceous rocks.

\section{ORGANIZATION OF THE CATALOG}

The catalog and the thin-section collection have been organized into nine units-five major units based on coal basins and States of the United States, one unit for foreign areas, and three units dealing with special coal sections, oil shale, and peat. In general, within the first five units, coals from the larger basins are grouped together. This system works adequately for the large number of slides from the Appalachian and the Interior basins and for the fewer slides from individual smaller Paleozoic coal basins in the United States. This arrangement accommodates virtually all bituminous or higher rank Paleozoic coal of the United States. However, the system loses some value for western coals, 
where rank is more obviously related to stage of metamorphism and where the coals generally are geologically younger. For these coal areas, a double division has been adopted. The Tertiary lignite of the Great Plains, Gulf Coast, and Mississippi Embayment has been grouped with the mostly Cretaceous-age subbituminous- and bituminous-rank coal of the Rocky Mountain States. The Cretaceous and Tertiary coals of the western coastal States and Alaska have been grouped together irrespective of rank.

The only individual States represented in two different major regions are Kentucky and Arkansas. Eastern Kentucky is included under the heading "Appalachian Basin," and western Kentucky, under the heading "Interior Basin." Arkansas is listed under "Other Paleozoic Basins" and "Rocky Mountains And Lignite Areas." A regional sequence of States, rather than an alphabetical sequence, has thus been established. Each State or part of a State has been given a specific letter designation, generally an abbreviation similar to that used by the U.S. Postal Service. The abbreviation is used to prefix specific slide-box numbers for items included from within each State.

Within each State, the primary listing is according to the name of the coal bed. Unidentified coal beds are listed alphabetically by county; miscellaneous items that are not easy to identify are always listed last. All miscellaneous slides, even if poorly labeled, have been preserved because their identities may be established later, and this information would increase their usefulness.

Materials of foreign origin are alphabetized according to country and locality. Numbered boxes of slides from foreign sources are indicated by a three-letter abbreviation. Of historic interest is one thin section, and the remaining small fragment of coal from which it was prepared, obtained on Admiral Byrd's 1937 Antarctic Expedition. Although not extensive, the foreign examples provide comparative petrographic information about many coal-producing areas in the world. A single capital letter combined with lowercase letters is used to prefix box numbers for material in the special coal sections, oil shale, and peat.

The slides for each of the 65 specific area or subject categories are stored in numbered slide boxes, usually no more than 25 slides per box. Additions can be made within each of the various categories as need arises. This method of labeling and indexing should make the collection easy to consult and to maintain.

The collection was arranged and cataloged in detail because nearly all the preparations are from localities that are no longer accessible. Even if essentially similar coal specimens can be obtained, the preparation of thin sections of coal involves a specialized technique which is not easy to duplicate. Finally, most coal petrographers recognize the advantage of being able to review the preparations that have been available to previous investigators. 


\section{MISCELLANEOUS ABBREVIATIONS}

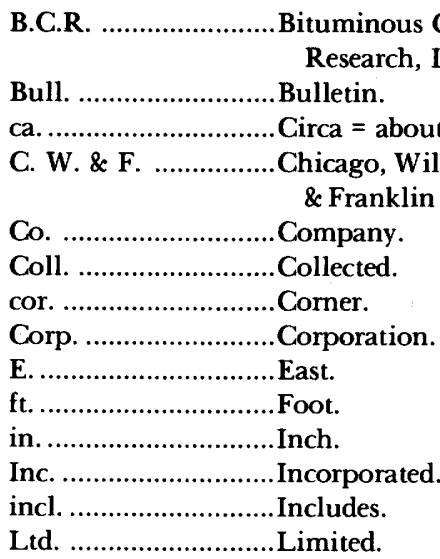

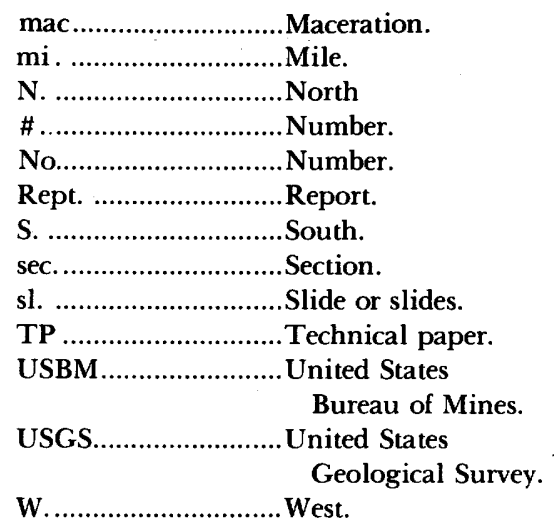

\section{UNIT 1. APPALACHIAN BASIN \\ OHIO}

OH 1; 22 sl. Brookville Coal. Crow mine, McArthur, Vinton County (middle bench, 9 sl.; upper bench, 5 sl.).

Clarion Coal. Sandy Run, Vinton County (8 sl.).

OH 2: 23 sl. Middle Kittanning Coal. Castner mine, Steubenville Deep Shaft Coal Co., Jefferson County. (Hydrogenation study. Lumps selected by J. M. S., Oct. 1944.)

OH 3; 23 sl. Middle Kittanning Coal (No. 6). Tryangle mine, Wallace Coal Co., $4 \mathrm{mi} \mathrm{N}$. of Roseville, Muskingum County. (Hydrogenation study. Sections from four 4-in. lumps. See USBM Bull. 550, 1956.)

$\mathrm{OH} \quad 4$; 18 sl. Middle Kittanning Coal. Motor Sales \#12 mine, Frazier, Muskingum County.

$\mathrm{OH} \quad 5 ; 9$ sl. Lower Kittanning Coal (top canneloid bench). Sunnyhill strip mine, Rehoboth, Perry County (from J. M. S., 5/24/50).

$\mathrm{OH}$ 6; 25 sl. Lower Kittanning Coal (Middle Kittanning?). Ohio Land and Rail-

$\mathrm{OH} \quad 7 ; 25$ sl. way Co. mine, Perry County (coll. Feb. 1956).

$\mathrm{OH} \quad 8 ; 11 \mathrm{sl}$.

OH 9; 25 sl. Lower Kittanning Coal. East Canton, Stark County (column 1).

$\mathrm{OH} \quad 10 ; 12 \mathrm{sl}$.

OH 11; 25 sl. Meigs Creek (No. 9 coal-lower bench). Dexter City, Noble County

OH 12; 26 sl. (coll. Apr. 1952).

OH 13; $25 \mathrm{sl}$.

OH 14; 25 sl. Meigs Creek (No. 9-upper bench). Dexter City, Noble County (coll.

$\mathrm{OH} \quad 15 ; 13$ sl. Apr. 1952).

$\mathrm{OH} \quad 16 ; 20$ sl. Nelm's Coal. Cadiz, Harrison County.

OH 17; 25 sl. Pittsburgh Coal. Apex mine, Amsterdam, Harrison County. (Coal

OH 18; 25 sl. column coll. $7 / 27 / 38$ by G. C. Sprunk, all sectioned. See USBM

OH 19; 21 sl. Bull. 550, 1956.)

OH 20; 7 sl. No. 2 or Quakertown Coal. McKitterick Coal Co., Sell farm, sec. 32, Coal Township, Jackson County (from J. J. McKitterick, Feb. 1940). 
Ohio-Continued

$\mathrm{OH} 21 ; 25$ sl. Quakertown or No. 2 Coal. Welleston No. 2 mine, McKitterick Coal $\mathrm{OH}$ 22; 21 sl. Co., Milton Township, Jackson County. (Column sample sent 2/11/38. See USBM Bull. 550, 1956.)

OH 23; 25 sl. Sharon No. 1 Coal. Sun mine, McKitterick Coal Co., about $2 \mathrm{mi}$ $\mathrm{OH} 24 ; 22$ sl. W. of Jackson, Jackson County. (Column sample sent 2/11/38. See USBM Bull. 550, 1956.)

OH 25; 25 sl. Sharon No. 1 Coal. Jisco mine, Jackson Iron and Steel Co., 1 mi OH 26; 10 sl. SW. of Jackson, Jackson County. (Column sample sent Sept. 1937. See USBM Bull. 550, 1956.)

$\mathrm{OH} 27$; 11 sl. Mahoning County. Fiske mine, Canfield (from B. L. Day, Jr. of American Fire Clay \& Products Co., 10/17/38. 2 sl.).

OH 28; 15 sl. Lower Kittanning Coal. E. C. E. exchange samples, northern Ohio. (From A. P. Thompson, Eagle Picher Co., coll. by B. C. Parks, 11/21/52.)

OH $29 ; 23 \mathrm{sl}$.

5 sl. Waynesburg Coal. Near Somerton, Belmont County (coll. May 1951).

1 sl. Pittsburgh Coal.

1 sl. Flint Ridge Cannel Coal. (6.4 percent spores).

1 sl. Middle Kittanning. 3-in. cannel layer (coll. 1956).

3 sl. Lower Kittanning. Banded coal and bony partings ca. $3 \mathrm{mi} \mathrm{S}+2$ mi W. of Clarion, Vinton County (coll. May 1951).

3 sl. $\quad$ Redstone Coal. \#4 mine, Pomeroy, Meigs County.

1 sl. Meigs Creek Coal. Pittsburgh Consolidated strip mine, Harrison County.

3 sl. Sharon Coal. Massilon, Stark County (Bright coal, collected by W. P. Yant, USBM).

4 sl. Cannel coal. Warsaw, Coshocton County (coll. May, 1940).

1 sl. Cannel coal. Bedford, Bedford Township, Coshocton County.

\section{PENNSYLVANIA}

PA 1; $25 \mathrm{sl}$.

PA $2 ; 23$ sl.

PA 3; $25 \mathrm{sl}$.

PA $4 ; 25 \mathrm{sl}$.

PA $5 ; 22$ sl.

PA 6; 22 sl.

PA $7 ; 25$ sl.

PA 8; $25 \mathrm{sl}$.

PA $9 ; 6$ sl.

PA 10; $24 \mathrm{sl}$.

PA 11; $25 \mathrm{sl}$.

PA 12; 3 sl.

Brookville? Coal. \#13 of Graeber and Thiessen "Correlation Series." Sugar Camp Run, McCracken coal bank, 2 mi NE. of Brookville, Jefferson County.

Brookville Coal. \#11 of Graeber and Thiessen "Correlation Series." Country bank, Welsh Run, Jefferson County. (Brookville coal of Graeber; Brookville coal of Thiessen. Is this the same country bank as C Ser. \#19? See USBM Bull. 550, 1956.)

Brookville Coal. \#1 of Graeber and Thiessen "Correlation Series." Conifer \#1 mine, Brookville, Beaver Township, Jefferson County. ( $6 \mathrm{mi} \mathrm{SW}$. of Brookville type locality, ca. $1 / 4 \mathrm{mi} \mathrm{S}$. of Conifer, NE. of Beaver Run. See USBM Bull. 550, 1956; TP 590, 1939.)

Brookville Coal. \# 4 of Graeber and Thiessen "Correlation Series." McClellan coal bank, Pine Creek Township, near south Sulger, ca. $3 \mathrm{mi}$ E. of Brookville, Jefferson County. (Mercer or Brookville coal of Graeber; Brookville coal of Thiessen. See USBM Bull. 550, 1956.) 
Pennsylvania-Continued

PA 13; 25 sl.

PA 14; 10 sl.

PA $15 ; 23 \mathrm{sl}$.

PA 16; 24 sl.

PA 17; 9 sl.

PA $18 ; 12$ sl.

PA 19; 19 sl.

PA 20; 25 sl.

PA 21; 22 sl.

PA 22; $25 \mathrm{sl}$.

PA 23; $16 \mathrm{sl}$.

PA 24; $13 \mathrm{sl}$.

PA 25; $25 \mathrm{sl}$.

PA 26; 25 sl.

PA 27; $11 \mathrm{sl}$.

PA 28; 23 sl.

PA 29; 25 sl.

PA 30; 25 sl.

PA 31; 25 sl.

PA $32 ; 10 \mathrm{sl}$.

PA 33; $25 \mathrm{sl}$.

PA $34 ; 8 \mathrm{sl}$.

PA 35; 25 sl.

PA 36 ; 16 sl.

PA 37; $23 \mathrm{sl}$.

PA $38 ; 13$ sl.

PA $39 ; 25 \mathrm{sl}$. PA $40 ; 16 \mathrm{sl}$.

PA 4l; $24 \mathrm{sl}$. PA $42 ; 18 \mathrm{sl}$.

PA 43; $25 \mathrm{sl}$. PA 44; 7 sl.
Brookville Coal. Scotch Hill mine, Mercer County.

Clarion Coal. \#7 of Graeber and Thiessen "Correlation Series." Hanley mine \#2, Summerville, Jefferson County. (Clarion coal of Graeber; Clarion coal of Thiessen. See USBM Bull. 550, 1956.)

Clarion Coal. \#3 of Graeber and Thiessen "Correlation Series." Baughman coal bank, Rose Township, $6 \mathrm{mi} \mathrm{SW}$. of Brookville, Jefferson County. (Clarion coal of Graeber; Brookville coal of Thiessen. See USBM Bull. 550, 1956.)

Clarion Rider Coal. \#8 of Graeber and Thiessen "Correlation Series." Hanley mine \#2, Summerville, Jefferson County ( $1 \mathrm{ft}$ thick, $18 \mathrm{ft}$ above $\mathrm{C}$ Ser. \#5).

Clarion Coal. \#6 of Graeber and Thiessen "Correlation Series." Humphrey quarry at Brookville, Jefferson County (1 ft thick, 18 ft above C Ser. \#5).

Thick Freeport Coal. Harmar mine, Harmarville, Allegheny County. (See USBM TP 655, 1943.)

Lower Freeport Coal. \#9 mine, near Alverda, Indiana County. (See USBM TP 621, 1941.)

Upper Freeport Coal. Wildwood mine, Wildwood, Allegheny County. (Coll. by L. N. Plein, USBM, and H. J. O'Donnell, USBM, 6/20/32. See USBM TP 564, 1935.)

Lower Kittanning Coal. \#72 mine, near Johnstown, Cambria County. (Coll. by C. M. Stull, USBM, 10/16/36. See USBM TP 595, 1939.)

Lower Kittanning Coal. Cadogan mine, 1/4 mi S. of Cadogan, Armstrong County. (Coll. by G. C. Sprunk, 4/27/38. See USBM Bull. $550,1956$.

Lower Kittanning Coal. \#18 of Graeber and Thiessen "Correlation Series." Jones bank, Limestone Township, Clarion County. (From drift just above the top of the shaft of the Sutton \#9 mine. See USBM Bull. 550, 1956.)

Lower Kittanning Coal. Indian Creek \#l mine, Indian Head, Fayette County. (Coll. $5 / 17 / 38$ by G. C. Sprunk, USBM. See USBM Bull. $550,1956$.

Lower Kittanning Coal. \#22 of Graeber and Thiessen "Correlation Series." McGary \#2 mine, Stanton, Jefferson County. (Coal 34 in. thick. See USBM Bull. 550, 1956.)

Lower Kittanning Coal. \#10 of Graeber and Thiessen "Correlation Series." Hanley Clay mine \#3, Summerville, Jefferson County (20 in. thick; Lower Kittanning coal of Graeber, Lower Kittanning coal of Thiessen. See USBM Bull. 550, 1956.) 
Pennsylvania-Continued

PA 45; $25 \mathrm{sl}$.

PA $46 ; 25 \mathrm{sl}$.

PA $47 ; 8 \mathrm{sl}$.

PA $48 ; 25$ sl.

PA $49 ; 5$ sl.

PA 50; $25 \mathrm{sl}$.

PA $51 ; 9$ sl.

PA $52 ; 24 \mathrm{sl}$.

PA $53 ; 19$ sl.

PA 54; 25 sl.

PA 55; $10 \mathrm{sl}$.

PA 56; 24 sl.

PA 57; $23 \mathrm{sl}$.

PA 58; 23 sl.

PA 59; $25 \mathrm{sl}$.

PA 60; $24 \mathrm{sl}$.

PA 61; 25 sl.

PA 62; $24 \mathrm{sl}$.

PA 63; $25 \mathrm{sl}$.

PA 64; 25 sl.

PA $65 ; 25 \mathrm{sl}$.

PA 66; 5 sl.

PA $67 ; 25 \mathrm{sl}$.

PA 68; $25 \mathrm{sl}$.

PA 69; $25 \mathrm{sl}$.

PA 70; 25 sl.

PA 71; $5 \mathrm{sl}$.

PA $72 ; 22$ sl.

PA $73 ; 24$ sl.

PA $74 ; 23$ sl.

PA $75 ; 25 \mathrm{sl}$.

PA $76 ; 26 \mathrm{sl}$.

PA $77 ; 15$ sl.

PA 78; 25 sl.

PA 79; $25 \mathrm{sl}$.

PA 80; 22 sl.

PA 81; $25 \mathrm{sl}$.

PA $82 ; 5$ sl.

PA $83 ; 14 \mathrm{sl}$.
Middle Kittanning Coal. \#23 of Graeber and Thiessen "Correlation Series." McGarey \#1 mine, Stanton, Jefferson County. (Middle Kittanning of Thiessen. See USBM Bull. 550, 1956.)

Upper Kittanning Coal. \#73 mine near Johnstown, Cambria County. (Coll. by C. M. Stull, 5/6/37. See USBM TP 595, 1939.)

Upper Mercer? Coal. \#12 of Graeber and Thiessen "Correlation Series." Brocius bank, Mill Creek, Jefferson County.

Upper Mercer Coal. \# 24 of Graeber and Thiessen "Correlation Series." Hell's Hollow mine, $1 \mathrm{mi} \mathrm{W}$. of Mercer, Mercer County. (Type locality Upper Mercer coal, $2 \mathrm{ft} 7$ in. mined, from White's Mercer County Report, 2d Pa. Survey, v. QQQ-137. Upper Mercer of Graeber(?); Mercer coal of Thiessen.)

Mercer Coal. \#9 of Graeber and Thiessen "Correlation Series." Carrier coal bank, near Ross School, Brookville area, Jefferson County. (Mercer coal of Graeber; Mercer coal of Thiessen.)

Pittsburgh Coal. Experimental mine, $1 \mathrm{mi}$ S. of Bruceton, Allegheny County. (Sample \#2 sectioned $8 / 1 / 39$ by G. C. Sprunk and L. L. Hirst. See USBM TP 594. 1939; TP 622, 1941; TP 666, 1944.)

Pittsburgh Coal. Ronco mine, H. C. Frick Coal Co., Ronco, Greene County. (Sample 1, location in mine 2d main butt, left heading. Column sample coll. by G. C. Sprunk, and H. J. O'Donnell 10/12/42.)

Pittsburgh Coal. Ronco mine, H. C. Frick Coal Co., Ronco, Greene County. (Sample \#2 collected by Sprunk and O'Donnell, Oct. 1942.)

Pittsburgh Coal. Edenborn mine, at Edenborn, Fayette County. (See USBM TP 525, 1932.)

Pittsburgh Coal. Pittsburgh terminal \#9 mine, 1 mi S. of Avella, Washington County. (See USBM TP 571, 1936.)

Pittsburgh Rider Coal. Florence strip mine, near Burgettstown, Washington County.

Pittsburgh Coal. Jamison \#20 mine, Pleasant Unity, Westmoreland County. (Coll. by G. C. Sprunk and H. J. O'Donnell, 4/13/38. 4 sl.)

Pittsburgh Coal. Adams mine, Irwin, Westmoreland County. (Humic woody cannel coll. by G. C. Sprunk, 4/22/38. 3 sl. See USBM TP 642, 1942; TP 666, 1944.)

Pittsburgh? Coal. Adams mine, Irwin, Westmoreland County. (7 sl. Kiskiminetas boghead, cannel. See USBM TP 642, 1942; TP 666, 1944.) 
Pennsylvania-Continued

PA 84; 10 sl. "B” Coal Seam. Bernice White Ash mine, Bernice, 2 mi S. of Mildred, Sullivan County. ( 5 sl. vitrain; 2 sl. attrital coal, semianthracite lump samples; 3 sl. "C"' seam? Coll. 5/10/46.)

PA 85; 25 sl. Brookville Coal. \#.2 of Graeber and Thiessen "Correlation Series." PA 86; $26 \mathrm{sl}$. Mineweaser coal bank, $2 \frac{1}{2} \mathrm{mi} \mathrm{N}$. of Brookville, ca. $1 / 2 \mathrm{mi} \mathrm{W}$. of north fork Redbank Creek, Eldred Township, Jefferson County. (Probably Brookville coal of Graeber; Brookville coal of Thiessen. See USBM Bull. 550, 1956.)

PA 87; 25 sl. Brookville Coal. \#15 of Graeber and Thiessen "Correlation Series." PA 88; 18 sl. Buzzard coal bank, S. side of Tunnel Hill, Brookville, Jefferson County (Brookville coal of Graeber; Brookville coal of Thiessen).

PA 89; 25 sl.

PA 90; 25 sl.

Brookville Coal. \#16 of Graeber and Thiessen "Correlation Series."

PA 9l; $6 \mathrm{sl}$.

Sutton \#.9 mine, Limestone Township, Clarion County. (Brookville coal of Graeber; Brookville coal of Thiessen. See USBM Bull. 550, 1956.)

PA 92; 19 sl. Brookville or Clarion Coal. \#19 of Graeber and Thiessen "Correlation Series." Ira Snyder country bank, Union Township, near Pine Grove School. (One of two thin coal beds between Little and Big Mill Creeks.)

PA 93; 23 sl. Clarion Coal (lower). \#17 of Graeber and Thiessen "Correlation PA $94 ; 5$ sl.

PA 95; 25 sl. PA 96; 13 sl.

Series." Shaft of Sutton \#9 mine, Limestone Township, Clarion County. (Clarion coal of Graeber; Clarion coal of Thiessen. See USBM Bull. 550, 1956.)

Lower Kittanning Coal. \#20 of Graeber and Thiessen "Correlation Series." Cowan mine, Roseville?, Union Township, Jefferson County. (Coal 30 in. thick, Roseville is in Tioga County; however this is at least 100 miles from Brookville, and Thiessen has Jefferson County in his report. See USBM Bull. 550, 1956.)

PA 97; 25 sl.

PA $98 ; 10 \mathrm{sl}$.

Lower Kittanning Coal. \#21 of Graeber and Thiessen "Correlation Series." Dehaven Coal Co. strip mine, Corsica, Clarion County (Coal 29 in. thick).

PA 99; 25 sl. Mercer or Brookville Coal. \#5 of Graeber and Thiessen "Correlation PA 100; 16 sl.

Series." Humphrey quarry, Brookville, Jefferson County (1 ft thick seam, $18 \mathrm{ft}$ below C Ser. \#6).

PA 101; 25 sl. Jefferson County. \#14 of Graeber and Thiessen "Correlation Series." PA 102; 20 sl. Chitestor bank, hill SE. of Brookville above Humphrey's quarry.

PA 103; 7 sl. Cannel Coal. Griest Mineral Co., Ebensburg, Cambria County. (From H. R. Griest, Gen. Manager Trust Bldg., no specific locality given.)

PA 104; 20 sl. Pittsburgh Coal. Farmers Market outcrop, near Monroeville, Allegheny County (3 sl.).

Pittsburgh Coal. Broughton, Allegheny County (bright attrital, 1 sl.).

Freeport Coal. Wildwood mine, Butler Consolidated Coal Co., Wildwood, Allegheny County. (Cannel boghead sample, $3 \mathrm{ft}$ above the thick Freeport, from Earl Maize, USBM. 7 sl.)

Non-banded Coal. $1 \mathrm{mi}$ S. of New Bethlehem, Armstrong County (2 sl.); 1 sl. marked northern Armstrong County.

Upper Kittanning Coal. Haek Brothers Coal Co., N. of Putneyville, Armstrong County ( 6 sl.). 
PA 105; 25 sl. Clarion Coal. Strip mine $1 \mathrm{mi} \mathrm{W.} \mathrm{of} \mathrm{Corsica,} \mathrm{Jefferson} \mathrm{County} \mathrm{(l} \mathrm{sl.).}$

Anthracite ("pock-marked") Coal. Near Mt. Carmel, Northumberland County. (Float near the top of the Pottsville Formation S. of Centralia fault. 1 sl.)

Dull banded and attrital coal. Worlds End State Park, Sullivan County (3 sl.).

Washington Coal. Road cut $2.1 \mathrm{mi}$ W. of Claysville, Washington County (1 sl.).

Upper Kittanning Cannel Coal. Bostonia mine (1 sl.).

Lower Kittanning Coal. Furnace Run \# 1 or \#6 mine, near Furnace Run, Armstrong County (9 sl.).

Cannel Coal? Paragon mine, Cannelton (2 sl.). (Possibly from Logan County Coal Corp. P.O. Yolyn, Logan County, W. Va.).

Carbonaceous sediments. Pennsylvania anthracite field, 1956 Samples from G. Wood. 7 sl.).

PA 106; 21 sl. Lower Freeport Coal. From Bethlehem Steel Co.

PA 107; 8 sl. Upper Freeport Cannel Coal. Somerset County (3 sl.).

Mississippian Coal. Leatherwood Station, New Bethlehem, Clarion County ( 5 sl.).

PA 108; 18 sl. Brookville Coal. (4/5/21, 1 sl.).

Thick Freeport Coal. (5 sl.).

Upper Freeport Coal. (4 sl.).

Pittsburgh Coal. (5 sl.).

Lower Freeport Coal. (Sewickley) Caldwell mine, 1/2 mi SE. of Curwensville, Clearfield County. (Allegheny Formation, 18 in. and 24 in. above floor, 2 sl.)

Pittsburgh Coal. E.C.E. exchange sample. Vesta mine, Jones and Laughlin Steel Corp., $1 \mathrm{mi}$ SW. of west Brownsville, Washington County (1 sl.).

\section{MARYLAND}

MD 1; 12 sl. Allegheny County. George's Creek coal basin, USBM drill core, hole 2GC, on Moores Run, 1.3 mi E. of Barton. (Upper and Lower Bakerstown and Upper Kittanning coals, hole started 2/15/45, completed 3/5/45. Sample coll. by J. J. Dowd. See USBM TP 725, 1949.)

MD 2; 12 sl. Lignitic wood, probably Cretaceous, Prince Georges Co.? (Near Washington, D.C., coll. by T. Stadnichenko. 2 sl.)

Sample 1-Good Luck Rd., Riverdale Heights. ${ }^{1}$

Sample 2-Muirkirk, Md. ${ }^{1}$

Sample 3-Nichols Ave., near Fort Grebble, Md. 83-F.

Sample 4-Virginia 83 (Va.).

Sample 5-Muirkirk Clay Pit, 83-M, Muirkirk, Md.

Sample 6-Good Luck Rd., Riverdale Heights. ${ }^{1}$

'Reserve pieces in box MD2 with sections. 


\section{WEST VIRGINIA}

WVa 1; 23 sl. WVa 2; $25 \mathrm{sl}$. WVa 3; $18 \mathrm{sl}$. WVa 4; 18 sl.

WVa $5 ; 25 \mathrm{sl}$. WVa $6 ; 23 \mathrm{sl}$. WVa $7 ; 9$ sl.

WVa $8 ; 25$ sl. WVa 9; $25 \mathrm{sl}$.

WVa 10; 25 sl. WVa 11; $15 \mathrm{sl}$.

WVa 12; 25 sl. WVa 13; 25 sl. WVa 14; 5 sl.

WVa $15 ; 24 \mathrm{sl}$. WVa 16; 25 sl. WVa 17; $5 \mathrm{sl}$.

WVa 18; 25 sl. WVa 19; 24 sl. WVa 20; $24 \mathrm{sl}$. WVa $21 ; 7 \mathrm{sl}$.

WVa 22; 25 sl. WVa 23; $25 \mathrm{sl}$. WVa 24; 19 sl. WVa 25; $25 \mathrm{sl}$. WVa 26; 24 sl. WVa 27; $25 \mathrm{sl}$. WVa 28; 16 sl.

WVa 29; 24 sl. WVa 30; 25 sl. WVa 31; 25 sl.

WVa $32 ; 18$ sl. WVa 33; 25 sl. WVa 34; 25 sl. WVa $35 ; 15 \mathrm{sl}$. WVa $36 ; 24 \mathrm{sl}$. WVa $37 ; 24$ sl. WVa $38 ; 5$ sl.

WVa $39 ; 22$ sl. WVa 40; $7 \mathrm{sl}$.

WVa $41 ; 25 \mathrm{sl}$. WVa $42 ; 25 \mathrm{sl}$. WVa $43 ; 7 \mathrm{sl}$.

WVa 44; $25 \mathrm{sl}$. WVa $45 ; 25$ sl. WVa $46 ; 10 \mathrm{sl}$.
Alma Coal. Spruce River \#4 mine, $1 / 2$ mi W. of Jeffrey, Boone County. (Coll. by L. N. Plein, 9/14/31. See USBM TP 562, 1935.)

Alma Coal. Red Jacket \#6 mine, Red Jacket, Mingo County. (Coll. by C. M. Stull, 7/21/35. See USBM Bull. 411, 1938.)

Bakerstown Coal. David \#l mine, Arthurdale Mining Co., Reedville, Preston County. (Column sample collected July 1952.)

Bakerstown Coal. Thomas \#23 mine, near Coketon, Tucker County. (Measured and sampled by A. E. Morrow, 2/9/40. See USBM TP 644, 1942.)

Beckley Coal. Winding Gulf \#l mine, Winding Gulf, Raleigh County. (Coll. by C. M. Stull, and A. E. Morrow, 11/21/35. See USBM Bull. 411, 1938.)

Cedar Grove Coal (top bench). Junior mine, Red Jacket, Mingo County. (Coll. by C. M. Stull, 10/3/35. See USBM Bull. 411, 1938.)

Cedar Grove Coal (bottom bench). Junior mine, Red Jacket, Mingo County. (Coll. by C. M. Stull, 10/2/35. See USBM Bull. 411, 1938; IC 7397, 1947.)

Chilton Coal. Boone County Coal Corp. \#2 mine, Monclo, Logan County. (Column sampled Sept. 1954. See USBM TP 542, 1932; TP $642,1942$.

Dorothy Coal. Carbon \#10 mine, Notomine, Kanawha County. (Coll. by C. M. Stull, 3/9/36. See USBM Bull. 411, 1938.)

Eagle Coal. Probably from prospect hole under \#7 mine, near Carbon, Kanawha County. (See USBM Bull. 550, 1956.)

Eagle Coal. Mallory \#3 mine near Mallory, Logan County. (Coll. by C. M. Stull, 3/18/36. See USBM Bull. 411, 1938.)

Eagle Coal. Kopperston mine, Kopperston, Wyoming County. (Column sample from Koppers Coal Co., Sept. 1942. See USBM Bull. $550,1956$.

\#5 Block Coal. American Rolling Mill Co. \#5 mine, 2 mi W. of Montcoal, Raleigh County. (See USBM Bull. 550, 1956.)

Upper Freeport Coal. \#21 mine at Dellslow, $7 \mathrm{mi}$ E. of Morgantown, Monongalia County. (Coll. by A. E. Morrow, 3/28/39. See USBM TP 621, 1941.)

Hernshaw Coal. Wharton \#2 mine, Eastern Gas and Fuel Associates, near Wharton, Boone County. 
West Virginia-Continued

WVa 47; $25 \mathrm{sl}$. WVa 48; 26 sl. WVa 49; 25 sl. WVa 50; 21 sl.

WVa $51 ; 16$ sl.

WVa 52; 25 sl. WVa 53; 5 sl.

WVa 54; $25 \mathrm{sl}$. WVa 55; $25 \mathrm{sl}$. WVa 56; $25 \mathrm{sl}$. WVa $57 ; 16 \mathrm{sl}$.

WVa 58; 25 sl. WVa 59; $25 \mathrm{sl}$. WVa $60 ; 25 \mathrm{sl}$. WVa $61 ; 25 \mathrm{sl}$. WVa $62 ; 6$ sl. WVa 63; $25 \mathrm{sl}$. WVa 64; 25 sl. WVa $65 ; 21 \mathrm{sl}$. WVa 66; 22 sl. WVa $67 ; 19$ sl.

WVa $68 ; 25 \mathrm{sl}$. WVa 69; $25 \mathrm{sl}$. WVa $70 ; \cdot 6 \mathrm{sl}$. WVa $71 ; 24$ sl. WVa 72; $25 \mathrm{sl}$. WVa 73; $11 \mathrm{sl}$. WVa 74; 24 sl. WVa $75 ; 9$ sl.

WVa $76 ; 18$ sl. Beckley Coal. Kitchekan mine, Barkers Ridge, $0.9 \mathrm{mi}$ NW. of junction of Mercer, Raleigh, and Wyoming County lines, Wyoming County. (Coll. by J. M. S. and B. W. Dean 10/23/73.)

WVa 77; 2 sl. Pocahontas No. 3 Coal. 1.0 mi NNE. of junction of Mercer, Raleigh, and Wyoming County lines, Raleigh County. (No. 2 core at 773 ft. collected by J. M. S. and B. W. Dean, Ken Englund, 10/23/73.)

WVa 78; 11 sl. No. 5 Block Coal. Harewood mine, 1.9 mi NE. of Boomer, Fayette County. (coll. by J. M. S. and B. W. Dean, 10/24/73.)

WVa 79; 25 sl. Boghead-Cannel Coal. Cedar Grove coal bed, Island Creek Coal Co. mine at Holden, Logan County. (Duplicating series, 9 sl. from side A; 8 sl. from side B; 8 sl. sample 2 from W. L. Long, 5/25/38.) 
West Virginia-Continued

WVa 80; 19 sl. Cannel Coal. Paulcille mine, Webster County, 31/2 mi up Elk River from Centralia (from Webster Sewell Coal. Co., 6/2/38. 8 sl.).

Fire Creek Coal, non-banded. Stanaford mine, Beckley, Raleigh County (4 sl.).

Meadow Branch. Myers mine, Berkeley County. (Coll. by A. T. Cross, 4/19/44. 5 sl.).

Cannel Coal? By-products \#1. (\#1 Thiessen, 2/23/27. 1sl.)

Monongalia County. E.C.E. exchange sample, Morgantown, 1955 (1 sl.).

\section{EASTERN KENTUCKY}

EKy $1 ; 18$ sl.

Elkhorn Coal. Consolidation \# 204 mine, Jenkins, Letcher County.

EKy $2 ; 8$ sl. (Coll. by R. D. Reeder, 1/16/30. See USBM TP 506, 1931; Bull. 550,

EKy 3; 25 sl. 1956.)

EKy $4 ; 12$ sl.

EKy 5; 20 sl.

EKy 6; 18 sl.

EKy 7; 13 sl.

EKy 8; 25 sl.

EKy 9; 25 sl.

EKy 10; 6 sl.

EKy 11; 12 sl.

EKy 12; 24 sl. EKy 13; 16 sl.

EKy $14 ; 24$ sl.

EKy 15; 24 sl.

EKy 16; 25 sl. EKy 17; 26 sl.

Elkhorn No. 3 Coal. Wheelwright mine, Wheelwright, Floyd County. (See USBM Bull. 550, 1956.)

Fire Clay \#4 Coal. Marlowe mine, Perry County, Cornettsville quadrangle (attrital coal below fire clay parting thin-sections contain spores and boghead algae, coll. by J. E. Johnston).

Hazard No. 4 Coal. Columbus \#4 mine, near Allais, Perry County. (See USBM TP 672, 1945.)

High Splint Coal. Closplint mine, Closplint, Harlan County. (Bed measured and sampled by A. E. Morrow, 9/28/40. See USBM TP 599, 1939.)

Lower Hignite Coal. Atlas mine, $7 \mathrm{mi} \mathrm{W}$. of Middlesboro, Bell County. (See USBM TP 634, 1942.)

EKy 18; 23 sl.

EKy 19; 25 sl.

EKy 20; 25 sl.

EKy $21 ; 25$ sl.

EKy 22; 22 sl.

EKy 23; 25 sl.

EKy 24; 6 sl.

Millers Creek Coal. Consolidation \#155 mine, $2 \mathrm{mi}$ NE. of Van Lear, Johnson County. (Coll. by L. N. Plein, 1/23/35. See USBM TP 572, 1937.)

EKy 25; 25 sl.

EKy 26; 17 sl.

EKy 27; 25 sl. EKy 28; 25 sl.

Pond Creek (Warfield) Coal. Majestic mine, Majestic, Pike County. (See USBM TP 596, 1939.)

Lower 18 in. Cannel Coal. Moore mine, Georges Creek, Breathitt County.

Straight Creek Coal. Big Jim mine, Blanche, Bell County. (Column sample collected by T. E. Gray, Washington, D.C. See USBM Bull. $550,1956$.

Taggart Coal. Lynch \#30 mines near Lynch, Harlan County. (See USBM TP 650,1943 .)

EKy 29; 18 sl. 
EKy 30; 17 sl. “Ash Check" Fire Clay Coal. (178 mm below clay parting. 1 sl.) 6 Blocks

Blue Gem Coal. Mammoth mine, near Gatliff, Whitley County. (Sample from W. A. Selvig, Jan. 1943. 5 sl.)

Cannel City Coal. Creek bed $6 \frac{1}{2} \mathrm{mi}$ N. of Cannel City, Morgan County (low banded part, 4 sl.).

Cannel City Coal. Cannel City, Morgan County. (Slides marked C. S. 1951. 7 sl. +6 reserve blocks.)

EKy 31; Cannel City Coal. 0.2 mi W of Cannel City, Morgan County. (Reserve blocks, column and reference log, collected N. of road along Stone Coal Fork by J. M. S., 5/17/53. 10 blocks.)

EKy 32; 15 sl. Low Splint Coal. Harlan County (Blocks 1 and 6, 8 sl.).

Harlan Coal. Harlan County (Block 4, 2 sl.).

Kellioka Coal. Harlan County (Blocks 7 and 8, 2 sl.).

Darby Coal. Harlan County (Block 2, 3 sl.).

(From Mr. Greenwald.)

EKy 33; 18 sl. Elkhorn \#1 Coal. Standard mine, Standard-Elkhorn Collieries, Inc., Floyd County. (Sample sent 5/20/38. See letter of $5 / 18 / 38,4$ sl.)

Elkhorn Coal (splint). Consolidation \# 204 mine, Consolidation Coal Co., Jenkins, Letcher County. (Sample sent 2/21/40. Sections 1-7 hydrogenation series, 8 sl. See USBM Bull. 550, 1956; TP 506, 1931.)

Elkhorn Coal. Elkhorn Coal Co. mine, Kona, Letcher County. (Coll. by J. E. Jones 6/16/45. 2 sl.)

Flag \#7 Coal (dull band). Kenmont Buckeye mine (317-340 mm below top. 4 sl. 1 block.)

EKy 34; 15 sl. Cannel 1 1/2 Coal. Worley \#4 mine, Stearns Coal and Lumber Co., Stearns, McCreary County (Hydrogenation study. Sampled by H. C. Fowler, Nov. 1939. 3 sl. See USBM TP 642, 1942.)

Hazard Coal. Block Coal Co., Lothair, Perry County. (3 sl., Hazard \#7 semi-splint block; 5 sl., Hazard \#4 bright coal block; 8 sl.).

Hazard \# 7 Coal. Perry County (semisplint, 4 sl.).

EKy 35; 15 sl. Cannel Coal (above Elkhorn). Pike Floyd mine, Betsy Layne, Floyd County (7 sl.).

Jellico Coal (cannel). Whitley County ( 3 sl.).

Pond Creek Coal (cannel). Stone \#3 mine, Eastern Coal Corp., near Stone, Pike County (2 sl.).

Straight Creek Coal. Hanby mine, Vox Ridge Fuel Co., Hanby, Bell County. (Hydrogenation study. Sampled by H. Fowler, Nov. 1939. 3 sl.)

EKy 36; 16 sl. Harlan County. Benham (2 sl.).

Letcher County. Cannel, Thornton Creek (2 sl.)

Morgan County. Cannel-boghead, Piedmont mine, Caney. (Hydrogenation study. Sample from Mr. Walter Barker of Caney, Dec. 1938. 12 sl.) 
Eastern Kentucky-Continued

EKy'37: 20 sl. Morgan County. Wrigley quadrangle (coal and shale, numbered slides, SHP 101, H66, H38, 12 sl.).

Pike County. C-9-58 at 365-366 ft (5 sl.).

Oak Creek Mine. Marlowe Coal Co. (Aug. 1950, 1 sl.).

Commercial Cannel. From E. Ky.? (1 sl.).

Cannel coal. EKy. field, By-products Co. ( sl.).

EKy 38; 5 sl. Elkhorn No. 1 and 2 Coal (upper layer). Roadcut off U.S. 23/460 about $3 / 4 \mathrm{mi}$ NNE. of Boldman, Pike County. (Coll. by J. M. S., B. W. Dean, $4 / 23 / 74$.)

\section{VIRGINIA}

VA $1 ; 25 \mathrm{sl}$.

Upper Banner Coal. Clinchfield \#9 mine, Clinchco, Dickenson

VA 2; $25 \mathrm{sl}$.

County. (Coll. by C. M. Stull, 1/28/36. See USBM TP 584, 1938.)

VA $3 ; 13 \mathrm{sl}$.

VA $4 ; 25 \mathrm{sl}$.

Lower Banner Coal. Keen Mountain mine, $2 \mathrm{mi}$ W. of Hanger,

VA $5 ; 25 \mathrm{sl}$. Buchanan County. (Collected by H. Fowler, 7/18/38. See USBM TP VA $6 ; 14 \mathrm{sl}$. 616,1940 .)

VA $7 ; 25 \mathrm{sl}$.

VA $8 ; 25 \mathrm{sl}$.

VA $9 ; 4 \mathrm{sl}$.

VA $10 ; 25 \mathrm{sl}$.

Clintwood Coal. Buchanan \#1 mine, Big Rock, Buchanan County.

VA $11 ; 21 \mathrm{sl}$.

Taggart Coal. Dunbar mine, Dunbar, Wise County. (Coll. by R.

VA $12 ; 9$ sl.

VA $13 ; 24 \mathrm{sl}$.

VA $14 ; 25 \mathrm{sl}$.

VA $15 ; 25 \mathrm{sl}$.

VA $16 ; 9 \mathrm{sl}$.

D. Reeder, 1/13/30. See USBM Bull. 550, 1956.)

Taggart Coal. Roda \#3 mine, Roda, Wise County. (See USBM Bull. $344,1931$.

Miller Coal. (Hanging Rock, seam \# 1, \#5).

\section{NORTH CAROLINA}

NC 1; 27 sl. Upper Cummnock Coal. Carolina mine, $10 \mathrm{mi}$ N. of Sanford, Chatham County. (Coal \#17-H, sampled by Shields, Parks, and O'Donnell. See USBM Bull. 515, 1952.)

NC 2; 22 sl. Chatham County. Sanford. (USBM core drill, hole D-1; 13 sl., 873 ft 11 in. to $876 \mathrm{ft} 6$ in.; 3 sl., $880 \mathrm{ft} 3 \frac{1}{8}$ in. to $880 \mathrm{ft} 7^{3 / 4}$ in.; 6 sl., $912 \mathrm{ft} 11^{3 / 8}$ in.)

NC 3; 11 sl. Chatham County. Sanford. USBM core drill, hole D-2, July 1944; 3 sl. $1,505 \mathrm{ft} 3$ in. to $1,508 \mathrm{ft} 7$ in.; 8 sl., $1,508 \mathrm{ft} 9$ in. to 1,519 ft 6 in.)

NC 4; 25 sl. Chatham County. (USBM core drill, hole D-2, 3/4/44; $1,507 \mathrm{ft} 0$ in. to $1,519 \mathrm{ft} 4 \frac{3}{4}$ in.)

NC 5; 22 sl. Chatham County. USBM core drill, hole E-1 "Deep River"; 16 sl., $975 \mathrm{ft} 3 / 4$ in. to $983 \mathrm{ft} 2$ in.; 6 sl., $1,010 \mathrm{ft} 4$ in. to $1,012 \mathrm{ft} 10^{7 / 8} \mathrm{in}$.)

NC $6 ; 8$ sl. Chatham County. Sanford. (USBM core drill, hole E-2, 10/3/44; 1,887 ft $1 \frac{1 / 2}{2}$ in. to $1,931 \mathrm{ft} 2 \frac{1}{8}$ in.) 
North Carolina-Continued

NC 7; 10 sl. Chatham County. (Bituminous sample from G. H. Gunter, Deep River Coal Corp., Gulf, N.C., supposed to contain fossil fish. 6 sl. 3 blocks.)

Black Band Layer. Carolina mine, Deep River. (Sideritic nodule in the black band layer. 2 sl.)

Chatham County. Triassic Coal area. (Band 23 in. thick between two veins of coal in the Triassic coal area in Lee and Chatham Counties. 2 sl.)

\section{TENNESSEE}

TN 1; 25 sl. Suwannee Coal. E.C.E. exchange sample. Reel's Cove mine, Tenn. TN 2; 8 sl. Products and Chemical Corp., near Whitwell, Marion County (1951, 7 sl.).

Obion County. E.C.E. exchange sample ( 1 sl.).

\section{ALABAMA}

AL 1; 25 sl. AL 2; $25 \mathrm{sl}$. AL $3 ;$; 16 sl. America Coal. America \#3 mine, Walker County. (Column sample collected Apr. 1952.)

AL $4 ; 25$ sl. AL $5 ; 22$ sl.

Black Creek Coal. Empire mine, Empire, Walker County (Coll. by AL $6 ; 25$ sl.

AL $\quad 7 ; 25 \mathrm{sl}$. A. C. Richardson, 2/25/31. See USBM TP 531, 1932.)

AL $8 ; 11$ sl.

Blue Creek Coal. Sumter mine and Adger mine, Birmingham, Jefferson County. (Sample from Mr. C. S. Blair of Black Diamond Coal Co., Birmingham, Ala., March 1937. 8 sl. from Sumter mine; 17 sl. from Adger mine.)

AL 9; 20 sl. Fairview Coal. USBM, DH 6-30. St. Clair County. (Sec. 30, T. 15 S., R. 4 E. ISBM diamond drill test, logged $208 \mathrm{ft} 5$ in. to 211 ft 7 in. Cored $1 / 15 / 45$ by A. Neale, Project Engineer. See USBM TP 719, 1949.)

AL $10 ; 25$ sl. Mary Lee Coal. Flat Top mine, near Flat Top, Jefferson County. (See AL $11 ; 24 \mathrm{sl}$. AL $12 ; 25 \mathrm{sl}$. USBM TP 519, 1932.)

AL 13; 25 sl. AL 14; $25 \mathrm{sl}$. Pratt Coal. Wylam \#8 mine, $1 \mathrm{mi}$ W. of Wylam, Jefferson County. (Coll. by B. W. Gandrud, 12/17/31. See USBM TP 564, 1935; IC 7397, 1947.)

AL 15: $18 \mathrm{sl}$.

Cherokee County. Near Fort Payne. (Core drill FP-43 1 ft. 10 in. AL 16; $25 \mathrm{sl}$. AL $17 ; 4 \mathrm{sl}$. sec. 2, T. 7 S., R. 10 E., cored by D. M. Coulter, Project Engineer, 5/24/44. Depth $248 \mathrm{ft} 3$ in. to $240 \mathrm{ft} \mathrm{l}$ in. See USBM Bull. 550, 1956.) 
Alabama-Continued

AL 18; 6 sl. America Coal. (Lower bench). USBM? hole G=7-D, Walker County (102 ft $8 \frac{1}{2}$ in. 1 sl.).

St. Clair County. USBM hole 1-1. (sec. 1, T. 16 S., R. 3 E., 478 ft. 8 in. 1 sl.).

Tuscaloosa County. Phalen Shephard \#l core hole (sec. 35, T. 17 S., R. 9 W. about $1 \frac{1}{2} \mathrm{mi}$ SE. of Wiley. Attrital coal at $432.22 \mathrm{ft}$ depth, l sl.)

Lignite (2 sl.).

H-8 (1 sl.).

\section{UNIT 2. INTERIOR BASIN}

\section{WESTERN KENTUCKY}

WKy 1; $25 \mathrm{sl}$. WKy 2; 19 sl.

WKy 3; 25 sl. WKy 4; $27 \mathrm{sl}$.

WKy 5; $25 \mathrm{sl}$. WKy $6 ; 25 \mathrm{sl}$. WKy $7 ; 16$ sl. WKy $8 ; 15$ sl.

WKy 9; 23 sl.

WKy $10 ; 13$ sl.

WKy 11; 25 sl. WKy 12; $25 \mathrm{sl}$. WKy 13; $25 \mathrm{sl}$. WKy 14; $25 \mathrm{sl}$. WKy $15 ; 19 \mathrm{sl}$.

WKy 16; $11 \mathrm{sl}$.
\#1 Bed Coal. Bell \#1 mine, $3 \mathrm{mi}$ SW. of Sturgis, Crittenden County. (See USBM TP 628, 1941.)

\#1 Bed Coal. \#4 mine, near Sturgis, Crittenden County. (Column sample from S. Wood, Mid-Continent Coal and Transportation Co., Jan. 1941. See USBM Bull. 550, 1956.)

\#6 Coal. Williams \#4 mine, 21/2 mi NW. of Mannington, Hopkins County. (Hydrogenation study. Column sample coll. by G. J. Flynn, Jr., Washington D. C., 5/13/44. See USBM Bull. 550, 1956.)

\#9 Coal. Moss Hill \#9 mine, 8 mi E. of Morton's Gapp, Muhlenberg County. (Hydrogenation study. Sample coll. by G. J. Flynn, Jr., 5/6/44. Petrographic analysis made 4/19/45. See USBM Bull. $550,1956$.

\# 11 Coal. North Diamond \#2 mine, 21/2 mi NW. of Earlington, Hopkins County. (See USBM Bull. 550, 1956.)

\#12 Coal. East opening Norton \#1 mine, at Nortonville, Hopkins County. (Hydrogenation study. Sample coll. by G. J. Flynn, Jr. 5/9-11/44. Petrographic analysis made April 1945. See USBM Bull. 550,1956 .)

\#14 Coal (Green River). Green River mine, Mogg, Muhlenberg County. (Coll. by J. M. Geyer, 8/26/32. See USBM TP 564, 1935.)

\#14 Coal (Green River). Sentry mine, $7 \mathrm{mi} \mathrm{W}$. of Madisonville, Hopkins County. (Hydrogenation study 3/19/45. Sample coll. by G. J. Flynn, Jr. 4/17/44. See USBM Bull. 550, 1956.)

\#15 Coal. Bald Knob mine, $3 \mathrm{mi}$ E. of Nebo, Hopkins County. (Hydrogenation study. Sample coll. by G. J. Flynn, Jr. 4/17/44. Petrographic analysis made $3 / 19 / 45$. See USBM Bull. 550,1956 .) 


\section{INDIANA}

IN $1 ; 25 \mathrm{sl}$. Indiåna \#4 (Linton Block) Coal. Saxton mine, 1/2/2 mi N. of Terre

IN 2; $25 \mathrm{sl}$.

IN $3 ; 19$ sl.

IN $4 ; 25 \mathrm{sl}$.

IN $5 ; 25 \mathrm{sl}$.

IN $6 ; 21 \mathrm{sl}$.

IN $7 ; 10 \mathrm{sl}$.

Block Coal. Maumee Collieries Old Glory \#17 mine, Clay City, Clay County (semisplint and splint).

IN 8; 21 sl. Block Coal. Big Bend Coal Co. mine, Centerpoint, Clay County. (Upper and lower veins from Big Bend Coal Co.; sl. 1-12 from lower vein bright coal; sl. 13-21 from upper vein, semisplint coal.)

IN 9; 18 sl. Brazil Block Coal. Brazil, Clay County (5 sl.).

(Upper) Brazil Block Coal. Maumee Colleries Old Glory \#17 mine, Clay City, Clay County (4 sl.).

Daviess County. Nonbanded coal. (Ohio State Univ. Collection \#1669, recorded by Professor Orton, 1892. 3 sl.)

Indiana \#5 Coal. Enos mine, Enos Coal Mining Co., near Oakland City, Pike County. (Black sulfur. 4 sl.)

Minshall Coal. Old Glory \#17 mine, Clay City, Clay County (1 sl.). County? Bradway mine, 1 mi E. of Adsherville? (1 sl.).

\section{ILLINOIS}

IL $1 ; 16$ sl. Colchester (No. 2) Coal. Wilmington \#10 mine, Northern Ill. Coal Corp., $3 \frac{1}{2}$ mi W. of Wilmington, Will County. (Hydrogenation study. Column collected by T. E. Gray. Petrographic analysis made March 1946. See USBM Bull. 550, 1956.)

IL 2; 25 sl. Colchester (No. 2) Coal. W. G. Sutton Coal Company, $1 \mathrm{mi} \mathrm{N}$. of IL 3; 19 sl. Minonk, Woodford County, sec. 6, T. 28 N., R. 2 E. (Hydrogenation study. Complete column from Illinois Geol. Survey, Oct. 1940. See USBM Bull. 550, 1956.)

IL 4; 25 sl. Harrisburg (No. 5) Coal. Cedar Hill mine, Eagle Valley area, Gallatin IL 5; 25 sl. County. (Sec. 23, T. 10 S., R. 8 E. Complete column from L. S.

IL 6; 19 sl. Wood, Mid-Continent Coal \& Trans. Co., Belleville, Illinois. See USBM Bull. 550, 1956.)

.IL 7; 25 sl. Springfield (No. 5) Coal. Panther Creek \#4 mine, Panther Creek Mines, Inc., $3 \mathrm{mi}$ NW. of Springfield, Sangamon County, sec. 29, T. 16 N., R. $5 \mathrm{~W}$. (Hydrogenation study. Column sample coll. by T. E. Gray, 12/11/45. See USBM Bull. 550, 1956.)

IL 8; 25 sl. Herrin (No. 6) Coal. Darmstadt \#1 mine, at Marissa, St. Clair County IL 9; $25 \mathrm{sl}$. extending to Washington County. (One thin section of roof from II $10 ; 25$ sl. H. L. Storch, sectioned by A. L. Furno, Sept. 1950, analysis by H. IL $11 ; 25$ sl. J. O'Donnell. See USBM Bull. 550, 1956.)

IL 12; 25 sl. Herrin (No. 6) Coal. Fidelity \#11 strip mine, $3 \mathrm{mi} \mathrm{W}$. of DuQuoin, IL 13; 3 sl. Perry County, sec. 21, T. 6 S., R. 2 W. (Hydrogenation scudy. Column sample coll. by T. E. Gray, 12/21/45. Petrographic analysis made May 1946. See USBM Bull. 550, 1956.) 
Illinois-Continued

IL $14 ; 25 \mathrm{sl}$.

IL $15 ; 3$ sl.

Herrin (No. 6) Coal. Staunton \#7 mine, Consolidated Coal Company, $1 \mathrm{mi} \mathrm{NE}$. of Staunton, Macoupin County, sec. 21, T. 7 N., R. 6 W. (Hydrogenation study. Column sample coll. by T. E. Gray, 12/21/45. Petrographic analysis made July 1946. See USBM Bull. $550,1956$.

IL 16; 21 sl. Herrin (No. 6) Coal. Middle Grove strip mine, Midland Elec. Coal Corp., near Middle Grove, $7 \mathrm{mi} \mathrm{W}$. of Farmington, Fulton County, sec. 2, T. 8 N., R. 3 E. (Hydrogenation study. Column sample coll. by T. E. Gray, 12/8/45. Petrographic analysis made Sept. 1946. See USBM Bull. 550, 1956.)

IL $17 ; 25 \mathrm{sl}$.

Herrin (No. 6) Coal. Orient \#l mine, $4 \mathrm{mi} \mathrm{N}$. of West Frankfort,

IL 18; $24 \mathrm{sl}$. Franklin County, sec. 10, T. 7 N., R. 2 E. (Measured and sampled

IL $19 ; 25 \mathrm{sl}$.

IL $20 ; 24$ sl.

IL $21 ; 12 \mathrm{sl}$.

IL $22 ; 5 \mathrm{sl}$. by E. R. Maize, 11/17/30. See USBM TP 524, 1932.)

IL 23; 19 sl. Harrisburg (No. 5) Coal. Thurmond Coal Co., Eagle Valley area, Gallatin County, sec. 18, T. 10 S., R. 8 E. (Slides labeled Hillcrest, Gallatin County.)

IL 24; 24 sl. Herrin (No. 6) Coal. (Briquette thin sections, USBM Lab No. C-31612, 19 sl; Lab. No. C-46294, 5 sl.)

IL 25; 15 sl. Springfield (No. 5) Coal. Panther Creek \#5 mine, in Springfield, Sangamon County, sec. 22, T. 16 N., R. 5 W. (2 sl.)

Herrin (No. 6) Coal. Zeigler \# 1 mine, near Zeigler, Franklin County, sec. 13, T. 7 S., R. 1 E. (2 sl.)

“Herrin (No. 6) Coal." Shelby (4 sl.) [The identification as Herrin (No. 6) Coal may be questioned if the coal is from Shelby County. Mines in the 1920's and 1930's produced Springfield (No. 5) Coal or Shelbyville Coal. From 1948 to 1958 Peabody \#17 mine produced No. 6 Coal from Shelby County, with the main operation and tipple in Christian County.]

Herrin (No. 6) Coal. Old Ben \#15 mine, near West Frankfort, Franklin County, sec. 35, T. 7 S., R. 2 E. (F-6-1, vitrain, 2 sl.)

Tartar Coal. (1 sl. and 1 polished section boghead bench and 2 sl. Tartar Coal) Outcropping, Fulton County, SW $1 / 1 / 4$ sec. 34, T. 6 N., R. 1 E. First reported boghead in Eastern Interior Basin. (See Am. Jour. Sci., v. 249, p. 444-450, 1951.)

Pope County. U.S. Dredge Jewett, specimen from river (cannel coal, from G. A. Pinneo; 1 sl. and 1 block specimen cannel coal). [Possibly coal from elsewhere in SE. Illinois or Indiana or barge refuse from the Ohio River.]

Vermilion County, Danville (United Electric, 1 sl.). [Probably No. 7 Coal. Numerous United Electric Mines produced No. 7 Coal in the 1920's and 1930's. The United Electric production of Herrin (No. 6) Coal appears to have been limited to 1960-65.] 


\section{IOWA}

IA $1 ; 25 \mathrm{sl}$.

IA $2 ; 16 \mathrm{sl}$.

IA $3 ; 25$ sl.

IA $4 ; 10 \mathrm{sl}$.

IA $5 ; 19 \mathrm{sl}$.

IA $6 ; 25 \mathrm{sl}$.

IA $7 ; 25$ sl.

IA $8 ; 14$ sl.

IA $9 ; 25 \mathrm{sl}$.

IA $10 ; 25 \mathrm{sl}$.

IA $11 ; 24 \mathrm{sl}$.

IA $12 ; 18 \mathrm{sl}$.

Lower Bed (Cherokee) Coal. Scandia \#4 mine, Scandia Coal Co. $1 / 2 \mathrm{mi} \mathrm{S}$. of Madrid, Boone County. (Sample from George J. Flynn, Jr., 1/11/41. See USBM Bull. 550, 1956.)

Mystic Coal. Sunshine No. 1 mine, Sunshine Coal Co., $4 \mathrm{mi} \mathrm{W.}$ of Centerville, Appanoose County. (Hydrogenation study. Column sample from G. J. Flynn, Jr., 12/19/40. See USBM Bull. 550, 1956.)

Nodaway Coal. Clarinda mine, $3 \mathrm{mi}$ W. of Clarinda, Page County. (Column sample from G. J. Flynn, Jr. 3/28/41. See USBM Bull. $550,1956$.

No. 3 Coal. Shuler No. 1 mine, $2^{\frac{1}{2}}$ mi NE. of Waukee. Dallas County. (Hydrogenation study. Column sample from G. J. Flynn, Jr., 3/17/41. See USBM Bull. 550, 1956.)

No. 3 Coal. Jensen mine, $8 \mathrm{mi}$ SW. of Fort Dodge, Webster County. (Column sample from G. J. Flynn, Jr., 4/9/41. See USBM Bull. $550,1956$.

Upper Cherokee Coal. Scandia Coal Co., Madrid, Boone County (1934, 3 sl.).

Upper Cherokee Coal. Urbandale Coal Co., Des Moines, Polk County (1934, 3 sl.).

Mammoth Coal. Marion County (3 si.).

Mystic Coal. McConnville Coal Co., Appanoose County (5 sl.).

No. 3 Coal. Shuler \#l mine, Shuler Coal Co., 21/2 mi NE. of Waukee, Dallas County (4 sl.).

\section{MISSOURI}

MO 1; 25 sl. Bevier Coal. Bee-Veer mine, Binkley Mining Co., near Jacksonville, Randolph County. (Hydrogenation study. Column sample from $T$. E. Gray, 12/14/45. Petrographic analysis, Oct. 1946. See USBM Bull. $550,1956$.

MO 2; 17 sl. Hickory County. Quincy. (Sample from Wallace Leiber, 8/20/46.)

Moniteau County. Simpson mine. (Cannel coal with sulfides-Pb, Zn, Fe. 2 sl.)

Morgan County. Barnett. (Sample from E. Athana. 14 sl.)

\section{KANSAS}

KS 1; 18 s1. Bevier Coal. \#18 mine, Pittsburgh and Midway Coal Co., West Mineral, Cherokee County. (Labeled "Limestone Seam," incl. 1 block.)

\section{UNIT 3. OTHER PALEOZOIC BASINS ARKANSAS (PALEOZOIC)}

ARp 1; 22 sl. Hartshorne Coal (lower). Johnson County.

\section{OKLAHOMA}

OK $1 ; 25$ sl.

Hartshorne Coal (upper and lower). \#17 mine, $4 \mathrm{mi} \mathrm{NE}$. of Bokoshe, OK 2; $25 \mathrm{sl}$. La Flore County. (See USBM TP 667, 1944.)

OK 3; 23 sl. 
Oklahoma-Continued

OK 4; 25 sl. Henryetta Coal. Atlas \#2 mine, near Henryetta, Okmulgee County.

OK $5 ; 25 \mathrm{sl}$. (See USBM TP 667, 1944.)

OK 6; 25 sl.

McAlester Coal. Dow \#10 mine, 8\%/2 mi E. of McAlester, Pittsburg

OK $7 ; 16 \mathrm{sl}$. County. (See USBM TP 667, 1944.)

OK $8 ; 2$ sl.

Coal County. Coalgate.

\section{MICHIGAN}

MI 1; 6 s1. Upper Pottsville Formation. Williamston, Ingham County. (Shale, found as lens between coal beds from upper Pottsville Formation near Williamston from S. G. Burgquist of Michigan State College, 1935. 5 sl.)

Impure Coal (slate?). E.C.E. exchange sample. Davidson mine, near Iron Mountain, Iron County. (Precambrian, See Tyler, Barghoorn and Barrett, GSA Bull. 68, 1957, 1 sl.)

\section{RHODE ISLAND}

RI 1; 4 sl. Newport County. USBM drill cores. (1 sl., hole \#1, 53 in. below $258 \mathrm{ft} 6$ in.; 2 sl., hole $\# 2,38$ in. below $69 \mathrm{ft} 0$ in., and $18 \mathrm{in}$. below $164 \mathrm{ft} 0$ in.; 1 sl., hole \#3, 12 in. below $352 \mathrm{ft} 0$ in.; cored Apr.-June 1945. See USBM RI-4276; 1948.)

\section{UNIT. 4. ROCKY MOUNTAINS AND LIGNITE AREAS ARKANSAS (LIGNITE)}

ARli 1; 22 sl. Clay County. Lignite, Jennings property, Crowley's Ridge, $6 \mathrm{mi} \mathrm{W}$ and $2 \mathrm{mi} \mathrm{S}$. of Pollard, (SE $\mathrm{SE}^{1 / 4} \mathrm{NW}^{1 / 1 / 4}$ sec. 6, T. 20 N., R. 7 E.; 10 lump samples from N. F. Williams of Arkansas State Geol. Survey, each about 4 in. square, from each 6 in. of bed thickness. Sections by A. L. Furno, analysis by H. J. O'Donnell. See USBM Buli. 482, 1950. 6 sl.)

Poinsett County. Lignite, Van Hoozer property, Bolivar Creek near Crowley's Ridge, $2 \mathrm{mi} \mathrm{N}$. + $1 \mathrm{mi} \mathrm{E}$. of Harrisburg. (NE $1 / 2 \mathrm{SE}^{\frac{1}{2}} \mathrm{sec}$. 7, T. 11 N., R. 4 E.; 10 lump samples from N. F. Williams of Arkansas State Geol. Survey, each about 4 in. square, from each 6 in. of bed thickness. Sections by A. L. Furno, analysis by H. J. O'Donnell. See USBM Bull. 482, 1950. $16 \mathrm{sl}$.)

ARli 2; 25 sl. ARli 3; 25 sl. Dallas County. Lignite, Manning strip mine, American Dyewood ARli 4; 25 sl. ARli 5; $18 \mathrm{sl}$. ARli 6; 27 sl. Co., $2 \mathrm{mi} \mathrm{S}$. of Manning. (Upper $6 \mathrm{ft}$ of $6 \mathrm{ft}$ to $8 \mathrm{ft}$ bed from $B$. C. Parks, H. J. O'Donnell and H. B. Foxhall, 9/10/47. See USBM Bull. 482, 1950.)

Hot Springs County. Lignite, outcrop on Two Mile Creek $2 \mathrm{mi}$ SE. of Malvern. (Bed about 18 in. thick sampled 9/10/47, by Parks, O'Donnell, and Foxhall, sections from small lumps by A. L. Furno, March 1948. See USBM Bull. 482, 1950.)

ARli 7; 25 sl. Ouachita County. Lignite, Garnet mine. (Near center of sec. 12, T. 12 S., R. 18 W.; slides from lumps marked "top of bed" represent first 10 in. from top down; those marked "second below the top," the third 10 in.; and slides marked "bottom" represent the lowest 10 in. of bed, 40 in. average thickness. Sampled by W. M. Wigel, Northern Pacific Railroad Co., 10/24/36.) 
Arkansas-Continued

ARli 8; 25 sl.

Ouachita County. Lignite, Old Big House mine, $5 \mathrm{mi} \mathrm{N}$. of Lester.

ARli 9; 25 sl.

(Column sample from B. C. Parks, H. J. O'Donnell, and H. B. Foxhall, 9/11/42. Originally Lester Lignite. See USBM Bull. 482, 1950.)

ARli 10; 7 sl. Hot Springs County. Lignite, Station 1. (Benzene-alcohol extraction. 3 sl.)

Ouachita County. Lignite, Old Big House mine, $5 \mathrm{mi} \mathrm{N}$. of Lester (4 sl.).

\section{MONTANA}

MT 1; $25 \mathrm{sl}$. MT 2; 25 sl.

Mammoth Coal. Gantar mine, Klein, $8 \mathrm{mi} \mathrm{SE}$. of Roundup, Musselshell County. (Hydrogenation study. Column sample from L. L. Hirst, 8/10/39. See USBM Bull. 550, 1956; TP 642, 1942.)

MT 3; 27 sl.

MT 4; 25 sl.

MT 5; $25 \mathrm{sl}$.

MT 6; $25 \mathrm{sl}$.

MT 7; 25 sl.

MT 8; 22 sl.

MT 9; $10 \mathrm{sl}$.

MT 10; 25 sl. MT $11 ; 25 \mathrm{sl}$. MT 12; 25 sl. MT 13; 3 sl.

MT 14; 25 sl. MT 15; 25 sl. MT 16; 3 sl.

Rosebud Coal. Colstrip mine, 1/2/2 mi SE. of Colstrip, Rosebud County. Hydrogenation study. Column sample from L. L. Hirst, 8/8/39. See USBM TP 622, 1941; Bull. 550, 1956.)

MT 17; 25 sl. MT 18; 25 sl. MT 19; 25 sl. MT 20; 25 sl. MT 21; 19 sl. MT 22; $14 \mathrm{sl}$.

Rosebud Coal. Colstrip mine, $1 \frac{1}{2} \mathrm{mi}$ SE. of Colstrip, Rosebud County. (Hydrogenation study. Sections of float and sink fraction from $\mathrm{J}$. M. S., Nov., 1944.)

Roundup Coal. Prescott mine, 1/8 mi S. of Roundup, Musselshell County. (Hydrogenation study. Column sample from L. L. Hirst, 8/11/39. See USBM Bull. 550, 1956; TP 642, 1942.)

Sand Coulee Coal. Giffin \#1 mine, $4 \mathrm{mi} \mathrm{SE}$. of Stockett, Cascade County. (Column sample from L. L. Hirst, 8/14/39. See USBM Bull. 550, 1956.)

\#3 Coal. Smith mine, $3 / 4$ mi E. of Washoe, Carbon County. (Hydrogenation study. Column sample from L. L. Hirst, $8 / 15 / 39$. See USBM Bull. 550, 1956; TP 642, 1942; TP 666, 1944.)

Above Mammoth Bed. Rider Coal, Musselshell County. (Vitrain, from H. C. Fowler, 1946. 3 sl.)

Roundup Coal. Musselshell County. (Subbituminous A, Paleocene. Fort Union Formation. 10 sl.)

Carter County. Ekalaka Hills. (Analcite in coal bed, Paleocene. 1 sl.)

MT 23; 3 sl. Anderson and Dietz No. 1 Coal. Decker mine, near Decker, about $20 \mathrm{mi} \mathrm{N}$. of Sheridan, Big Horn County (Woody specimen about $10 \mathrm{ft}$ below the top of a $52 \mathrm{ft}$ seam, $5 \mathrm{ft}$ below a parting. Coal thickness $38 \mathrm{ft}$ to $42 \mathrm{ft}$ elsewhere. Collected by J. M. S., USGS, 7/25/73.)

\section{NORTH DAKOTA}

ND 1; 25 sl. Beulah Coal. Beulah, Mercer County. (Commercially delivered lignite, crushed coal. Oct. 1953.)

ND 2; 23 sl. Beulah? Coal. Knife River mine, Knife River Mining Co., near Beulah, Mercer County. (Hydrogenation study. Lignite sections 2-25. See USBM TP 622, 1941; TP 642, 1942; Bull. 550, 1956.) 
North Dakota-Continued

ND 3; 25 sl. Beulah-Zap Coal. Beulah mine, Knife River Coal Co., 2 mi NE. of Beulah, Mercer County. (Hydrogenation study. Lignite from A. Koth, 5/21/42, petrographic analysis Oct. 1946. Layers 1-7. See USBM Bull. 550. 1956.)

ND 4; 25 sl. Beulah-Zap Coal. As above (layers 7-13).

ND 5;

ND 6; 8 sl. Beulah-Zap Coal. As above (layers 13, 14).

ND 7 ;

ND 8;

ND 9; 25 sl.

Burleigh Coal. Burleigh strip mine, $3 \mathrm{mi} \mathrm{E}$. of Wilton Burleigh

ND 10;25 sl. County. (Hydrogenation study. Lignite. See USBM Bull. 550, 1956.)

ND $11 ; 25$ sl.

ND 12; 25 sl.

ND $13 ; 25 \mathrm{sl}$.

ND 14; $10 \mathrm{sl}$.

ND 15; $25 \mathrm{sl}$. ND $16 ; 25 \mathrm{sl}$.

ND 17; $25 \mathrm{sl}$.

ND 18; $10 \mathrm{sl}$.

ND 19; $25 \mathrm{sl}$. ND 20; 25 sl.

ND 21; $25 \mathrm{sl}$.

ND 22; $25 \mathrm{sl}$.

ND 23; 19 sl.

ND 24; $10 \mathrm{sl}$.

ND 25; $15 \mathrm{sl}$.

ND 26; 25 sl.

ND 27; 3 sl.

ND 28; $25 \mathrm{sl}$.

ND 29; $25 \mathrm{sl}$.

ND 30; $25 \mathrm{sl}$.

ND 31; $25 \mathrm{sl}$.
Burleigh Coal. Wilton mine, Truax-Traer Lignite Co., 1 mi E. of Wilton, Burleigh County. (Hydrogenation study. Lignite from Alfred Kelsven, May 1939. Fifty $1 \frac{1}{2}$ in. lump samples +1 slide insect remains. See USBM TP 642, 1942; Bull. 550, 1956.)

Custer Coal. Stevens Bros. Coal Co. mine, Carrison, McClean County. (Hydrogenation study, May 1939.)

Garrison Dam Coal. Garrison Dam, Mercer County (Lignite drill core).

Harmon Coal. USGS, DH-5. "Medicine Pole Hills" about $13 \mathrm{mi}$ SW. of Bowman, Bowman County ( $\mathrm{SW}^{1 / 4} \mathrm{SE}^{1 / 4}$ sec. 35, T. 131 N., R. 140 W. See USGS Trace Elements Investigations Rept. 572, 1955.)

Harmon Coal. USGS, DH-6. "Medicine Pole Hills" about $13 \mathrm{mi}$ SW. of Bowman, Bowman County. ( $\mathrm{SE}^{1 / 4 \mathrm{NE}^{1 / 4} \mathrm{sec} .}$ 2, T. 130 N., R. 140 W. See USGS Trace Elements Investigations Rept. 572, 1955.)

Harmon Coal. USGS, DH-9. "Medicine Pole Hills" about $13 \mathrm{mi}$ SW. of Bowman, Bowman County (NW'1/4NE1/4 sec. 2, T. 130 N., R. 140 W. See USGS Trace Elements Investigations Rept. 572, 1955.)

Haynes Coal. Dakota Collieries Co. mine, $1^{3 / 4} \mathrm{mi} \mathrm{NE}$. of Haynes, Adams County. (Lignite, column sample of bed thickness from A. Koth, Univ. N. Dak., 5/22/42. See USBM Bull. 550, 1956.)

Haynes Coal. As above. 
North Dakota-Continued

ND 32; $25 \mathrm{sl}$. ND $33 ; 3$ sl.

ND 34; $25 \mathrm{sl}$.

ND $35 ; 25 \mathrm{sl}$.

ND $36 ; 25 \mathrm{sl}$.

ND $37 ; 9$ sl.

ND 38; 25 sl.

ND 39; 25 sl.

ND $40 ; 24$ sl.

ND $41 ; 25 \mathrm{sl}$.

ND $42 ; 23 \mathrm{sl}$.

ND 43; $25 \mathrm{sl}$.

ND $44 ; 12 \mathrm{sl}$.

ND 45; $25 \mathrm{sl}$. ND $46 ; 10 \mathrm{sl}$.

ND $47 ; 15 \mathrm{sl}$.

ND $48 ; 25 \mathrm{sl}$.

ND 49; $25 \mathrm{sl}$.

ND $50 ; 25 \mathrm{sl}$.

ND 51; $25 \mathrm{sl}$.

ND 52; $25 \mathrm{sl}$.

ND 53; $25 \mathrm{sl}$.

ND 54; $25 \mathrm{sl}$.

ND 55; $25 \mathrm{sl}$.

ND $56 ; 12 \mathrm{sl}$.
Haynes Coal. Rupp Coal Co. mine near Haynes, Adams County. (Hydrogenation study. Lignite received May 1939. See USBM TP 642, 1942; Bull. 550, 1956.)

Indian Head Coal. Indian Head mine, Zap, Mercer County. (Commercially delivered lignite, crushed coal sections 31-50.)

Indian Head Coal. Indian Head mine, Zap, Mercer County. (Zap column lignite sample, Jan. 1951.)

Kincaid Coal. Kincaid mine, Columbus, Burke County. (E.C.E. exchange sample, lignite lump specimen June 1953.)

Lehigh Coal. Lehigh mine, Lehigh, $4 \frac{1}{2} \mathrm{mi}$ SE. of Dickinson, Stark County. (Hydrogenation study. Lignite from A. Koth, 5/23/42. Petrographic analysis Oct. 1946, layers 1-7. See USBM Bull. 550, 1956.)

Lehigh Coal. Lehigh, Stark County. (Delivered lignite, 1953.)

Noonan Coal. Baukol-Noonan mine, near Noonan, Divide County. (NW $1 \frac{1}{4}$ sec. 11, T. 162 N., R. 95 W. Lignite column sample from Arthur Koth, Univ. N. Dak., $5 / 25 / 42$, sectioned by L. L. Hirst, May 1943. See USBM Bull. 550, 1956.)

Noonan Coal. As above. (Hydrogenation study. Lignite representative of bed thickness from employees of Baukol-Noonan Lignite, Inc., 8/17/39. See USBM TP 642, 1942; Bull. 550, 1956.)

Quality Coal. Burlington mine, $2 \mathrm{mi} \mathrm{SW}$. of Burlington, Ward County. (Lignite from Arthur Koth, Univ. N. Dak., 5/26/42. See USBM Bull. 550, 1956.)
ND $57 ; 25 \mathrm{sl}$.

ND 58; $25 \mathrm{sl}$.

ND $59 ; 14 \mathrm{sl}$.

ND $60 ; 18 \mathrm{sl}$.
Velva Coal. Velva mine, Traux-Traer Coal Co., $10 \mathrm{mi}$ SW. of Velva, Ward County. (Hydrogenation study. Complete lignite column from L. C. Harrington, Univ. N. Dak., 11/5/39. See USBM TP 622, 1941; Bull. 550, 1956.)

Divide County. Baukol-Noonan mine near Noonan, Divide County. (Upper $2 \mathrm{ft}$ of $7 \mathrm{ft}$ thickness silicified, gymnospermous woodtracheids, humic infiltrated material-from Fowler 10/3/46 to USBM 12/20/46. 2 sl.)

McKenzie County. Shelly mine, $12 \mathrm{mi}$ NE of Watford City. (1 sl. fusain, 20-in. upper bench; 1 sl. attrital lignite from 9-in. middle bench; 1 sl. lignite from lower 15 -in. bench. Total 3 sl.) 


\section{North Dakota-Continued}

ND $60 ; 18$ sl. (cont.)
SD $1 ; 15 \mathrm{sl}$.

SD 2; 8 sl.

SD $3 ; 12$ sl.

SD 4; 23 sl.

SD 5; 22 sl.

SD 6; 20 sl.

SD 7; $20 \mathrm{sl}$.

SD 8; $23 \mathrm{sl}$.

SD 9; $25 \mathrm{sl}$.

SD 10; $25 \mathrm{sl}$.

SD 11; $25 \mathrm{sl}$.

SD 12; $25 \mathrm{sl}$.

SD 13; 25 sl.

SD 14; $25 \mathrm{sl}$.

SD 15; $25 \mathrm{sl}$.

SD 16; 12 sl.

SD 17; $18 \mathrm{sl}$.

SD 18; $21 \mathrm{sl}$.

SD 19; $25 \mathrm{sl}$.

SD 20; 25 sl.

SD 21; 25 sl.

SD 22; $24 \mathrm{sl}$.

SD 23; $25 \mathrm{sl}$.

SD 24; $23 \mathrm{sl}$.

SD 25; $24 \mathrm{sl}$.

SD 26; $24 \mathrm{sl}$.

SD 27; 14 sl.

SD 28; $15 \mathrm{sl}$.
Ward County. Quality Lignite mine, $9 \mathrm{mi} \mathrm{S}$. and $1 \frac{1}{2} \mathrm{mi} \mathrm{W}$. of Sawyer. (Lignitic "knotty" wood through 12-ft upper bench, humic infiltration. 2 sl.)

Ward County. Vix mine (SW $1 / \frac{1}{4}$ sec. 24, T. 152 N., R. 82 W. Lignite wood from lower $7 \frac{1}{2} \mathrm{ft}$ of the $14 \mathrm{ft}$ bed of Coteau coal.)

Ward County. (Anthraxylon from lignite, prepared for Kreulen. 3 sl.)

Williams County. Standard mine, $3 \mathrm{mi}$ E. of Williston. (Lignitic wood from Fowler 11/1/46: 2 sl. from 2-5 ft below top of $9-\mathrm{ft}$ coal; 1 sl. from lower $4 \mathrm{ft}$ of $7-\mathrm{ft}$ coal)

\section{SOUTH DAKOTA}

E and F Beds? USGS core, Homestake Mining Co., Riley \#4 claim, North Cave Hills, about 18 mi NNE. of Buffalo, Harding County. (Near center NW $1 / 4 \mathrm{NW}^{1 / 4} \mathrm{sec} .26, \mathrm{~T} .22 \mathrm{~N} ., \mathrm{R} .5 \mathrm{E}$. Includes 5 polished blocks. See USGS Trace Elements Investigations Rept. 480, 1956.)

Mendenhall Coal. Mendenhall adit, Mendenhall area, Southern Slim Buttes about $20 \mathrm{mi}$ SE. of Buffalo, Harding County. (NW/4 $\mathrm{SE}^{1 / 4} \mathrm{sec}$. 1, T. 17 N., R. 7 E. Abandoned strip pit. See USGS Trace Elements Investigations Rept. 572, 1955; 480, 1956.)

Upper Mendenhall Coal. USGS core, SD-19. Mendenhall area, Southern Slim Buttes, about $20 \mathrm{mi}$ SE. of Buffalo, Harding County. (SW 14 SW $^{1 / 1} / 4$ sec. 31 , T. 18 N., R. 8 E. See USGS Trace Elements Investigations Rept. 572, 1955; 480, 1956.)

Lower Mendenhall Coal. As above.

Two thin coals between the Lower Mendenhall and Upper Olesrud coals. Locality as above.

Upper Olesrud Coal. As above.

Olesrud Coal. As above.

Mendenhall Coal (Upper Rider). USGS, DH-8. Mendenhall area, Southern Slim Buttes, about 20 mi ESE. of Buffalo, Harding County.

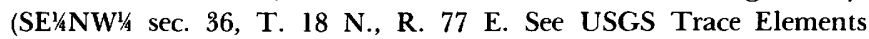
Investigations Rept. 572, 1955.)

Mendenhall Coal (Lower Rider). As above.

Mendenhall and Olesrud Coals. As above. 
SD 29; $26 \mathrm{sl}$

South Dakota-Continued

SD $30 ; 11 \mathrm{sl}$.

Olesrud Coal. As above.

SD $31 ; 25 \mathrm{sl}$.

SD 32; $23 \mathrm{sl}$.

Olesrud Coal. As above.

SD $33 ; 9$ sl.

SD $34 ; 25 \mathrm{sl}$.

SD $35 ; 25 \mathrm{sl}$.

SD $36 ; 21 \mathrm{sl}$.

SD $37 ; 25 \mathrm{sl}$.

Lower Olesrud Coal. As above.

SD $38 ; 6 \mathrm{sl}$.

Z-Bed. Adit, Southern Slim Buttes area; Harding County. (NE1/4SW1/4 sec. 2, T. 16 N., R. 5 E. Column sample. See USGS Trace Elements Investigations Rept. 480, 1956.)

SD $39 ; 12 \mathrm{sl}$.

Z-Bed. As above. (Lignitic wood with analcite.)

Z-Bed. Southern Slim Buttes area, Harding County. (NE $1 / 4 \mathrm{SW}^{1 / 4}$ sec. 2, T. 16 N., R. 5 E. See USGS Trace Elements Investigations Rept. $480,1956$.

SD 40; 4 sl. Harding County. Billy Dale No. 1 claim, ca. 2 mi SE. of Riley Pass, about $13 \mathrm{mi} \mathrm{N}$. of Buffalo, S. Dak., in the North Cave Hills. (Specimens from J. R. Gill Dec. 1954, 1 ft thick ore-grade uraniferous coal.)

SD $41 ; 26 \mathrm{sl}$.

Mendenhall Coal. USGS, DH-3, Mendenhall area, Southern Slim Buttes, about $20 \mathrm{mi}$ ESE. of Buffalo, Harding County. (SW $1 / 4 \mathrm{SE}^{1 / 4}$ sec. 36 , T. 18 N., R. 7 E. See USGS Trace Elements Investigations Rept. 480, 1956.)

SD 42; 21 sl. Mendenhall Coal. USGS, DH-4. As above, Harding County. (NW1/4SE $1 / 4$ sec. 36 , T. 18 N., R. 7 E.)

SD 43; $25 \mathrm{sl}$.

SD $44 ; 25 \mathrm{sl}$.

SD $45 ; 25 \mathrm{sl}$.

SD $46 ; 8 \mathrm{sl}$.

SD $47 ; 25 \mathrm{sl}$.

SD $48 ; 25 \mathrm{sl}$.

SD $49 ; 25 \mathrm{sl}$.

SD $50 ; 25 \mathrm{sl}$.

SD 51; $24 \mathrm{sl}$.

SD $52 ; 10 \mathrm{sl}$.

SD $53 ; 16 \mathrm{sl}$.

Olesrud Coal. USGS, DH-10. As above, Harding County. ( $\mathrm{NE}^{1 / 4} \mathrm{SE}^{1 / 4}$ sec. 12, T. 17 N., R. 7 E.)

SD $54 ; 18 \mathrm{sl}$.

Mendenhall Rider Coal. USGS, DH-16. As above, Harding County. (SW $1 \frac{1}{4} \mathrm{SE}^{1 / 4}$ sec. 36 , T. 18 N., R. 7 E.)

Mendenhall and Olesrud Coals. USGS, DH-17. As above, Harding County. (SW $1 / 4 \mathrm{SW}^{1 / 4}$ sec. 6 , T: 17 N., R. 8 E.)

SD $55 ; 18 \mathrm{sl}$.

Mendenhall and Olesrud Coal. USGS, DH-18. As above, Harding

SD $56 ; 20 \mathrm{sl}$.

County. (SE $1 / 4 \mathrm{NE}^{1 / 4} \mathrm{NE}^{1 / 4}$ sec. 1, T. 17 N., R. 7 E.)

SD $57 ; 10 \mathrm{sl}$.

Olesrud Coal. As above.

SD $58 ; 100 \mathrm{sl}$.

SD $59 ; 74 \mathrm{sl}$.

Olesrud Coal. USGS, DH-19. Bar H area, Northern Slim Buttes, about $20 \mathrm{mi}$ E. of Buffalo, Harding County. (NW'/4NE $1 / 4 \mathrm{NE}^{1 / 4} \mathrm{sec}$. 32, T. 19 N., R. 8 E. See USGS Trace Elements Investigations Rept. $572,1955$.

SD 60; 16 sl. Harding County. USGS, DH-21. As above. (Center of SW $1 / 4$ sec. 8, T. 18 N., R. 8 E.) 
South Dakota-Continued

SD 61; 8 sl. Harding County. USGS, DH-22. As above. (SW'/1/4 NW/4, sec. $8, T$. 18 N., R. 8 E.)

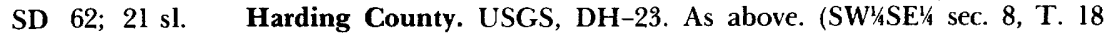
N., R. 8 E.)

SD 63; 22 sl. Harding County. USGS, DH-24. As above. (Center of NE $1 / 4$ sec. 5,

SD $64 ; 12 \mathrm{sl}$. T. 18 N., R. 8 E.)

SD $65 ; 22 \mathrm{sl}$.

Harding County. USGS, DH-25. As above. $\left(\mathrm{NE}^{1 / 4} \mathrm{NE}^{1 / 4}\right.$ sec. 3 , T. 18

SD $66 ; 17 \mathrm{sl}$. N., R. 8 E.)

SD $67 ; 24 \mathrm{sl}$.

SD $68 ; 7 \mathrm{sl}$.

Harding County. USGS, DH-26. As above. (Center of NW1/4 sec. 35,

SD $69 ; 4$ sl. T. 19 N., R. 8 E.)

SD 70; 11 sl. Harmon Coal. USGS, DH-11. Lodgepole Hills area, about 2 mi S.

Harding County. USGS, DH-27. As above. (NW'1/4NE $1 / 4$ sec. 35, T. 19 N., R. 8 E.) of Lodgepole Post Office, Perkins County. (Near center SE $1 / 4 \mathrm{NE}^{1 / 4}$ sec. 19, T. 21 N., R. 12 E.)

SD 71; 7 sl. Harmon Coal. USGS, DH-12. As above, Perkins County. (NW1/4SE $1 / 4$ sec. 19, T. 21 N., R. 12 E.)

SD 72; 3 sl. Harmon Coal. USGS, DH-13. As above, Perkins County. (NW/1/4 NE $1 / 4$ sec. 19, T. 21 N., R. 12 E.)

\section{WYOMING}

WY 1; 25 sl.

Carney Coal. Acme mine near Acme, $8 \mathrm{mi} \mathrm{N}$. of Sheridan, Sheridan

WY 2; $25 \mathrm{sl}$.

County. (Coll. by L. L. Hirst and W. S. Landers, $8 / 21 / 39$. See USBM

WY 3; $25 \mathrm{sl}$.

Bull. 550, 1956.)

WY 4; 25 sl.

WY 5; $21 \mathrm{sl}$.

WY 6; 25 sl.

WY $7 ; 25$ sl.

WY $8 ; 25$ sl.

WY 9; $25 \mathrm{sl}$.

WY $10 ; 10 \mathrm{sl}$.

WY 11; $25 \mathrm{sl}$.

WY 12; $25 \mathrm{sl}$.

WY 13; 25 sl.

WY 14; 25 sl.

WY 15; $25 \mathrm{sl}$.

WY 16; 19 sl.

WY 17; $25 \mathrm{sl}$.

WY 18; 25 sl.

WY 19; $25 \mathrm{sl}$.

WY 20; $3 \mathrm{sl}$.

WY 2l; $4 \mathrm{sl}$.

Hanna \#2 Coal. Hanna \#4 mine, Hanna, Carbon County. (Hydrogenation study. Sections of every third inch of column sample from W. S. Landers, Golden, Colo., 11/2/40. See USBM Bull. 550, 1956.)

Kemmerer \#1 Coal. Brilliant \#8 mine, 14 mi S. of Kemmerer, Lincoln County. (Coll. by L. L. Hirst, 9/1/39. See USBM Bull. 550, 1956.)

Kemmerer \#5 Coal. Gomer \#5 mine, near Frontier, $5 \mathrm{mi} \mathrm{N}$. of Kemmerer, Lincoln County. (Coll. by L. L. Hirst, 8/31/39. See USBM TP 642, 1942; Bull. 550, 1956.)

WY 22; $25 \mathrm{sl}$. WY 23; $25 \mathrm{sl}$. WY 24; $25 \mathrm{sl}$. WY 25; $25 \mathrm{sl}$.

Mammoth Coal. Elkol mine, Kemmerer Coal Co., $8 \mathrm{mi} \mathrm{S}$. of Kemmerer, Lincoln County. (Hydrogenation study. Float and sink fractions sample from J. M. S., Oct. 1944.)

WY 26; $8 \mathrm{sl}$.

Mammoth Coal. Elkol mine, $8 \mathrm{mi}$ S. of Kemmerer, Lincoln County. (Hydrogenation study. Incomplete column sample from L. L. Hirst, 9/1/39. See USBM Bull. 550, 1956.) 
Wyoming-Continued

WY 27; 25 sl. WY 28; $25 \mathrm{sl}$. WY 29; 25 sl. WY $30 ; 25 \mathrm{sl}$. WY $31 ; 18$ sl. WY $32 ; 18$ sl. WY $33 ; 12$ sl.

WY $34 ; 25$ sl. WY $35 ; 25 \mathrm{sl}$. WY $36 ; 25 \mathrm{sl}$. WY $37 ; 21 \mathrm{sl}$.

WY $38 ; 25$ sl. WY 39; $25 \mathrm{sl}$. WY 40; $25 \mathrm{sl}$. WY 41; $25 \mathrm{sl}$. WY 42; 4 sl.

WY 43; $25 \mathrm{sl}$. WY 44; $25 \mathrm{sl}$. WY $45 ; 19 \mathrm{sl}$. WY $46 ; 25$ sl. WY 47; $25 \mathrm{sl}$. WY 48; 25 sl. WY 49; $23 \mathrm{sl}$. WY 50; $25 \mathrm{sl}$. WY 51; 25 sl. WY 52; $25 \mathrm{sl}$. WY 53; 27 sl. WY 54; 25 sl. WY 55; $25 \mathrm{sl}$. WY 56; $25 \mathrm{sl}$. WY 57; $11 \mathrm{sl}$. WY 58; $25 \mathrm{sl}$. WY 59; $25 \mathrm{sl}$. WY 60; $26 \mathrm{sl}$. WY 61; $25 \mathrm{sl}$. WY 62; $25 \mathrm{sl}$. WY 63; $25 \mathrm{sl}$. WY 64; $25 \mathrm{sl}$. WY 65; $25 \mathrm{sl}$. WY 66; $25 \mathrm{sl}$. WY 67; $25 \mathrm{sl}$. WY 68; $22 \mathrm{sl}$. WY $69 ; 5 \mathrm{sl}$.

WY 70; $25 \mathrm{sl}$. WY 71; $25 \mathrm{sl}$. WY 72; $25 \mathrm{sl}$. WY 73; $23 \mathrm{sl}$.
Monarch Coal (lower bench). Monarch \#45 mine, $10 \mathrm{mi} \mathrm{N}$. of Sheridan, Sheridan County. (Hydrogenation study. Collected by V. F. Parry, 3/4/39. See USBM TP 622, 1941; Bull. 550, 1956.)

Rock Springs \#1 Coal. "D" mine, $2 \mathrm{mi}$ N. of Superior, Sweetwater County. (Coll. by L. L. Hirst, V. F. Parry, and W. S. Landers, 8/24/39. See USBM Bull. 550, 1956.)

Rock Springs \#l Coal. Winton mine near Winton, Sweetwater County. (Coll. by L. L. Hirst, and W. S. Landers, 3/28/39. See USBM Bull. 550, 1956.)

Rock Springs \# 3 Coal. Gunn Quealy mine, $1 / 2$ mi W. of Quealy, Sweetwater County. (Coll. by L. L. Hirst, 8/31/39. See USBM TP 642, 1942; Bull. 550, 1956.)

Rock Springs \#7 Coal. D. O. Clark mine, $2 \mathrm{mi}$ N. of Superior, Sweetwater County. (Coll. by L. L. Hirst. V. F. Parry, and W. S. Landers, 8/25/39. See USBM Bull. 550, 1956.)

Rock Springs \#7//2 Coal. Winton mine near Winton. Sweetwater County. (Coll. by L. L. Hirst, and W. S. Landers, 8/28/29. See USBM Bull. 550, 1956.)

Rock Springs \#.9 Coal. D. O. Clark mine, $2 \mathrm{mi} \mathrm{N}$. of Superior, Sweetwater County. (Coll. by L. L. Hirst, V. F: Parry, and W. S. Landers, 8/25/39. See USBM Bull. 550, 1956.)

Rock Springs \#15 Coal. D. O. Clark mine, $2 \mathrm{mi} \mathrm{N}$. of Superior, Sweetwater County. (Coll. by L. L. Hirst, V. F. Parry, and W. S. Landers, 8/25/39. See USBM Bull. 550, 1956.)

Roland and Smith Coal. Wyodak mine, 6 $6 \frac{12}{2} \mathrm{mi}$ E. of Gillette, Campbell County. (Hydrogenation study. Sections every fourth inch of column sample. Total 566 sections. Collected by L. L. Hirst, V. F. Parry, and W. S. Landers, 9/17/39. See USBM TP 642, 1942; Bull. $550,1956$.

6 Foot Coal? M. C. mine, 6 mi NNE. of Glenrock, Converse County. (2 sl., wood from top 8 in.; 1 sl., crushed fusain from top 8 in.; 2 sl., coal from middle of bed, from H. C. Fowler, USBM, 12/6/46.)

Washakie Coal. Hudson \#2 mine, $6 \mathrm{mi}$ S. of Hudson, Fremont County. (Coll. by L. L. Hirst, V. F. Parry, and W. S. Landers, 8/22/39. See USBM Bull. 550, 1956.) 
WY 74; 14 sl.

Wyoming-Continued

Willow Creek Coal. USBM, DH 1-6, about $8 \mathrm{mi} \mathrm{N}$. of Kemmerer, Lincoln County. (586 ft E of $\mathrm{W}^{1 / 4}$ cor. sec. 6, T. $24 \mathrm{~N}$., R. $115 \mathrm{~W}$. Complete column top bed 1 ft 11 in. from A. L. Toenges, Sept. 1942. Sections of alternating inches. See USBM TP 673, 1945.)

WY 75; $26 \mathrm{sl}$. WY 76; 25 sl. WY 77; 9 sl.

WY 78; $25 \mathrm{sl}$. WY 79; $25 \mathrm{sl}$. WY 80; $25 \mathrm{sl}$. WY $81 ; 21 \mathrm{sl}$.

WY 82; $25 \mathrm{sl}$. WY 83; 25 sl. WY $84 ; 25$ sl. WY $85 ; 18 \mathrm{sl}$.

WY 86; 17 sl.

WY $87 ; 17$ sl.

WY 88; 24 sl. WY 89; 19 sl. WY 90; $25 \mathrm{sl}$. WY 9l; $13 \mathrm{sl}$. WY 92; 10 sl. WY 93; $13 \mathrm{sl}$. WY 94; $11 \mathrm{sl}$. WY 95; $13 \mathrm{sl}$.

WY 96; 25 sl. WY 97; $25 \mathrm{sl}$. WY 98; $24 \mathrm{sl}$. WY 99; $18 \mathrm{sl}$. WY 100; $14 \mathrm{sl}$.

WY 101; $10 \mathrm{sl}$.

WY 102; 23 sl. WY 103; $25 \mathrm{sl}$. WY 104; 13 sl.
Willow Creek Coal. USBM, DH 1-6, as above (bottom bed).

Willow Creek Coal. USBM, DH 1-12, about $8 \mathrm{mi} \mathrm{N}$. of Kemmerer Lincoln County. ( $30 \mathrm{ft} \mathrm{N}, 600 \mathrm{ft}$ W of $\mathrm{E}^{1 / 4}$ cor. sec. 12, T. $23 \mathrm{~N}$., R. 116 W. Sample from A. L. Toenges. See USBM TP 673, 1945.)

Willow Creek Coal. USBM, DH 1-13, about $8 \mathrm{mi} \mathrm{N}$. of Kemmerer, Lincoln County. $\left(1,168 \mathrm{ft} W\right.$ of the $E^{1 / 4}$ cor. sec. 13, T. $23 \mathrm{~N}$., R. $116 \mathrm{~W}$. Main bed core drill from A. L. Toenges. See USBM TP 673, 1945.)

Willow Creek Coal. USBM, DH 1-24, about $8 \mathrm{mi} \mathrm{N}$. of Kemmerer, Lincoln County. (1,699 ft E. of center sec. 24, T. 23 N., R. 116 W. Main bed column from A. L. Toenges. See USBM TP 673, 1945.)

Willow Creek Coal. USBM, DH 2-12, about $8 \mathrm{mi} \mathrm{N}$. of Kemmerer, Lincoln County. (NE cor. sec. 12, T. 23 N., R. 116 W. Complete bottom bed column from A. L. Toenges, Sept. 1942. See USBM TP 673, 1945.)

Willow Creek Coal. USBM, DH 2-13, about $8 \mathrm{mi} \mathrm{N}$. of Kemmerer, Lincoln County. (1,461 ft W. of SE. cor. sec. 13, T. 23 N., R. 116 W. Complete column of bottom $3 \mathrm{ft}$ of bed from A. L. Toenges, Sept. 1942. Sections of alternate inches. See USBM TP 673, 1945.)

Willow Creek Coal. USBM, DH 2-13, as above (top bed).

Willow Creek Coal. USBM, DH 2-31, about $8 \mathrm{mi} \mathrm{N}$. of Kemmerer, Lincoln County. (2,353 ft E. of NW. cor. sec. 31, T. 24 N., R. 115 W.; lower bed, sections 1-19; middle main bed, sections 20-64; upper bed, sections 65-91. Complete column from A. L. Toenges, May 1943. 46 mounted blocks sectioned June 1943. See USBM TP 673, 1945.)

Willow Creek Coal. USBM, DH 3-13, about $8 \mathrm{mi} \mathrm{N}$. of Kemmerer, Lincoln County. (1,584 ft E. of $\mathrm{N}^{1 / 4}$ cor. sec. 13, T. $23 \mathrm{~N}$., R. 116 W.; Main bed core from A. L. Toenges, USBM, sectioned Aug. 1942. See USBM TP 673, 1945.)

Willow Creek Coal. USBM, DH 3-13, as above (bottom bed column not complete).

Willow Creek Coal. USBM, DH 4-13, about $8 \mathrm{mi} \mathrm{N}$. of Kemmerer, Lincoln County. (NW cor. $\mathrm{SE}^{1 / 4} \mathrm{SE}^{1 / 4}$ sec. 13, T. 23 N., R. $116 \mathrm{~W}$. Complete column of main bed top bench from A. L. Toenges, Oct. 1942. Sections of alternating inches. See USBM TP 673, 1945.)

Willow Creek Coal. USBM, DH 4-13, as above (middle bench). Willow Creek Coal. USBM, DH 4-13, as above (bottom bench). 
WY 105; $25 \mathrm{sl}$. WY 106; 25 sl. WY 107; 25 sl.

WY108; 25 sl. WY109; 22 sl.

WY110; 6 sl.

WY111; 25 sl. WY 112; $25 \mathrm{sl}$. WY113; 25 sl. WY114; 26 sl.

WY115; 25 sl. WY 116; 25 sl. WY117; 23 sl. WY 118; 23 sl.

WY119; 25 sl. WY 120; $14 \mathrm{sl}$.

WY 121; 25 sl. WY 122; 5 sl.

WY 123; $25 \mathrm{sl}$. WY 124; $19 \mathrm{sl}$. WY 125; 22 sl. WY 126; 17 sl. WY 127; 7 sl.

WY 128; $10 \mathrm{sl}$.

WY 129; $25 \mathrm{sl}$. WY 130; 9 sl.

WY 131; 13 sl.

WY 132; $25 \mathrm{sl}$. WY 133; 24 sl.

WY 134; 7 sl.

WY 135; $16 \mathrm{sl}$

\section{Wyoming-Continued}

Willow Creek Coal. USBM, DH 4-31, about $8 \mathrm{mi} \mathrm{N}$. of Kemmerer, Lincoln County. $(1,720 \mathrm{ft}$ E., $1,320 \mathrm{ft} N$. of SW. cor. sec. 31, T. 24 N., R. 115 W Complete column from A. L. Toenges, Aug. 1943. See USBM TP 673, 1945.)

Willow Creek Coal. USBM, DH 4=31, as above.

Fremont County. Continuation of Squaw Butte \# 1-2 core (Depth 6,110-6,145 ft. Polished block sample from W. R. Keefer, 4/10/60.)

Johnson County. De Smet core GS $\perp 2 A$, near Lake De Smet. (Near

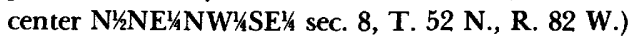

Johnson County. De Smet core A-6, near Lake De Smet. (Near center

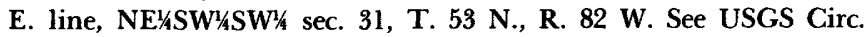
228, 1953. Healy? coal.)

Johnson County. De Smet core GS-1, near Lake De Smet. (Near center $S^{1 / 2 N E} / 1 / N^{1} / 4$ sec. 1, T. 52 N., R. 83 W. See USGS Circ. 228 , 1953. Healy? coal.)

Johnson County. De Smet core GS-3, near Lake De Smet. (Near center $\mathrm{W}^{1 / 2} \mathrm{SW} \mathrm{W}^{\mathrm{T} / 4} \mathrm{sec} .6$, T. 52 N., R. 82 W. See USGS Circ. 228, 1953. Healy? coal.)

Johnson County. De Smet core GS-6, near Lake De Smet. (Near center $S W / 2 W^{1 / 1 / 4}$ sec. 1, T. 52 N., R. 83 W. See USGS Circ. 228, 1953. Healy? coal.)

Niobrara County. Union Agate Well, Continental Oil Co., Little Buck Creek Field. (Coal or coaly shale from Dave Love, 11/22/49.)

Sweetwater County. USBM DH-1. Red Desert, about $30 \mathrm{mi}$ NNW of Wamsutter. ( $\mathrm{SW}^{1 / 4} \mathrm{sec}$. 24, T. 24 N., R. 96 W. See Trace Elements Investigations Rept. 390, 1953.)

Sweetwater County. USBM DH=2. Red Desert, about $30 \mathrm{mi} \mathrm{NNW}$. of Wamsutter. (Sec. 16, R. 24 N., R. 95 W. See Trace Elements Investigations Rept. 390, 1953.)

Sweetwater County. USBM DH-3. Red Desert, about $30 \mathrm{mi}$ NNW. of Wamsutter. (SW1/4 sec. 21, T. 24 N. R. 95 W. See Trace Elements Investigations Rept. 390, 1953.)

Sweetwater County. USBM DH-4, Red Desert, about $30 \mathrm{mi}$ NNW. of Wamsutter. (SE ${ }^{1 / 4} \mathrm{NW}^{1 / 4}$ sec. 28 , T. 24 N., R. 95 W. See Trace Elements Investigations Rept. 390, 1953.)

Sweetwater County. USBM DH-5. Red Desert, about 30 mi NNW. of Wamsutter (NW1/4 sec. 15, T. 24 N., R. 95 W. See Trace Elements Investigation Rept. 390, 1953.)

Sweetwater County. USBM DH-6. Red Desert, about 30 mi NNW. of Wamsutter. (SW1/4NE $1 / 4$ sec. 10, T. 24 N. R. 95 W. See Trace Elements Investigations Rept. 390, 1953.) 
Wyoming-Continued

WY 136; 11 sl. Sweetwater County. USBM DH-7. Red Desert, about $30 \mathrm{mi}$ NNW. of Wamsutter. (SW1/4 sec. 20, T. 24 N., R. 95 W. See Trace Elements Investigations Rept. 390, 1953.)

WY 137; 6 sl. Sweetwater County. USBM DH-8, and USBM DH-9. Red Desert, about $80 \mathrm{mi} \mathrm{NNW}$. of Wamsutter. (DH-8, NE $\frac{1}{4} \mathrm{SW}^{1 / 4}$ sec. 22, T. 24 N., R. 96 W.; DH-9, sec. 2, T. 24 N., R 95 W. See Trace Elements Investigations Rept. 390, 1953.)

WY 138; 25 sl. Sweetwater County. USBM DH-10. Red Desert, about $30 \mathrm{mi}$ NNW. WY 139; 20 sl. of Wamsutter. (Sec. 18, T. 23 N., R. 94 W. See Trace Elements Investigations Rept. 390, 1953.)

WY 140; $25 \mathrm{sl}$. WY 141; $25 \mathrm{sl}$. WY 142; 9 sl.

Sweetwater County. USBM DH-11. Red Desert, about $30 \mathrm{mi}$ NNW. WY 143; 25 sl. WY 144; 25 sl. WY 145; $25 \mathrm{sl}$ WY 146; 3 sl. WY $147 ; 10 \mathrm{sl}$. of Wamsutter. (NW $1 / 4 \mathrm{NW}^{1 / 4}$ sec. 27, T. 23 N., R. 94 W. See Trace Elements Investigations Rept. 390, 1953.)

Sweetwater County. USBM DH-49. Red Desert, about $10 \mathrm{mi}$ NNE. of Wamsutter. (NWT/4NW1/4 sec. 13, T. 21 N., R. 93 W. See Trace Elements Investigations Rept. 390, 1953.)

Sweetwater County. USBM DH=53. Red Desert, about $10 \mathrm{mi} \mathrm{N}$. of Wamsutter. (NE $1 / 4 N^{1} 1 / 4$ sec. 16, T. 21 N., R. 93 W. See Trace Elements Investigations Rept. 390, 1953.)

USBM DH-66, about $3 \mathrm{mi}$ NE. of Wamsutter. (NW/1/4SE $1 / 4 \mathrm{sec}$. 13, T. 20 N., R. 94 W. See Trace Elements Investigations Rept. 390, 1953.)

USBM DH-68, about $4 \mathrm{mi} \mathrm{NW}$. of Wamsutter. (SW/4 SW'1/4 sec. 5 , T. 20 N., R. 94 W. See Trace Elements Investigations Rept. 390, 1953.)

USBM DH-70, about 6 mi NE. of Wamsutter. (SE $1 / 4 \mathrm{NW}^{1 / 4} \mathrm{sec}$. 15, T. 20 N., R. 93 W. See Trace Elements Investigations Rept. 390, 1953.)

WY 148; 25 sl. WY 149; $25 \mathrm{sl}$. WY 150; $25 \mathrm{sl}$. WY 15l; $25 \mathrm{sl}$. WY 152; 14 sl. WY 153; 6 sl.

Sweetwater County. Luman \#1 coal, USBM DH-72. Red Desert about $30 \mathrm{mi} \mathrm{NNW}$. of Wamsutter. (SE $1 / 1 / \mathrm{NW}^{1 / 4} \mathrm{sec}$. 15, T. $24 \mathrm{~N}$., R. 95 W. See USGS Trace Elements Investigations Rept. 527, 1955.)

Willow Creek Coal. USBM DH 3-31, near Kemmerer, Lincoln County. (2,115 ft E., 1,320 ft S. of NW. cor. sec. 31, T. 24 N., R. 115 W., lower bed. 3 sl. See USBM TP 673, 1945.)

Carbon County. E. C. E. exchange sample, $4 \mathrm{mi}$ from Walcott (1 sl.).

(2 sl., vitrain from J. M. S. 1/10/47).

WY 154; 20 sl. Armstrong Coal. Big Horn mine, about $8 \mathrm{mi} \mathrm{N}$. of Sheridan, Sheridan County. (NE $1 / 4$ sec. 22, T. 57 N., R. 84 W. Collected by B. E. Law, USGS, $5 \mathrm{ft}$ above the base, 3/10/73. Sample LBT-3, 6 sl.)

Monarch Coal. As above. (9 $\mathrm{ft}$ above the base of the $17-\mathrm{ft}$ coal. Sample LBT-2, 5 sl.)

Monarch Coal. As above. ( $15 \mathrm{ft}$ above the base of the $22-\mathrm{ft}$ coal. Sample LBT-1, 3 sl.)

“Wood" coal bed. Negro Draw. (NE'// sec. 8, T. 56 N., R. 82 W. Collected by W. C. Culbertsen, USGS, 6 sl.) 


\section{IDAHO}

ID $1 ; 25 \mathrm{sl}$.

ID 2; $25 \mathrm{sl}$.

ID 3; $25 \mathrm{sl}$.

ID $4 ; 19 \mathrm{sl}$.

ID $5 ; 18 \mathrm{sl}$.

ID $6 ; 12 \mathrm{sl}$.

Cassia County. USGS, DH-2, Goose Creek, about $17 \mathrm{mi} \mathrm{SW}$. of Oakley. (Sec. 24, T. 16 S., R. 21 E. Lignite ground samples. See USGS Circ. 343, 1954; Trace Elements Investigations Rept. 669, 1957.)

Cassia County. USGS, DH-2. As above. (Lignite.)

Cassia County. USGS, DH-2, DH-3A. As above. (Lignite ground samples. 11 sl., from hole-2; 7 sl., from hole-3A.)

Cassia County. USGS, DH-3A. Goose Creek, about $17 \mathrm{mi} \mathrm{SW}$. of Oakley. (Sec. 14, T. 16 S., R. 21 E. See Trace Elements Investigations Rept. 669, 1947; USGS Circ. 343, 1954.)

E. C. E. exchange sample A-47097 (1 sl.).

\section{COLORADO}

CO 1; 25 sl.

CO 2; 25 sl.

CO 3; 25 sl.

CO $4 ; 16 \mathrm{sl}$.

Fox Hill Coal. Pike View mine, $6 \mathrm{mi} \mathrm{N}$. of Colorado Springs, El Paso County. (Hydrogenation study. Column sample from C. L. Ross, Golden, Colo., 11/22/40. 88 sections +2 blocks. 3 sections of minus 100-mesh digested uraninite. See USBM Bull. 550, 1956.)

CO $5 ; 25 \mathrm{sl}$.

CO 6; 25 sl.

CO $7 ; 5$ sl.

CO $8 ; 25 \mathrm{sl}$.

CO 9; 25 sl.

CO 10; $25 \mathrm{sl}$.

CO $11 ; 8 \mathrm{sl}$.

CO 12; $20 \mathrm{sl}$.

Hart Coal. Baker mine, Moffat County.

Lenox Coal. Lenox mine, Edna Coal Co., 5 mi NW. of Oak Creek, Routt County. (Column from W. L. Crentz, 7/22/48, all sectioned. See USBM Bull. 550, 1956.)

Oliver (No. 10) Coal. Oliver mine, $2 \mathrm{mi}$ E. of Somerset, Gunnison County. (Sullivan Co. under contract from the Oliver Coal Co., 1,500 ft E. of the NW. cor. sec. 22, T. 13 S., R. 90 W. Cored 6/4/46, logged $268 \mathrm{ft} 2$ in. to $287 \mathrm{ft} 5 \mathrm{in}$. Thickness of seam $19 \mathrm{ft} 3 \mathrm{in}$.)

CO 13; 25 sl. CO $14 ; 25$ sl. CO $15 ; 25 \mathrm{sl}$. CO 16; 25 sl. CO $17 ; 8 \mathrm{sl}$. CO 18; $6 \mathrm{sl}$.

Walsen Bed? Cameron mine, Robinson No. 4, Farr, Huerfano County (NW $1 \frac{1}{4} \mathrm{NW}^{1 / 4} \mathrm{NW}^{1} / 4 \mathrm{NE}^{1 / 4} / 4$ sec. 21, T. 28 S., R. $66 \mathrm{~W}$. Thin sections from 2 lumps Apple Coal or Niggerheads from J. M. S., July 1945.)

CO 19; 25 sl.

CO 20; 25 sl.

CO 21; 25 sl.

CO 22; 25 sl.

CO 23; 25 sl.

CO $24 ; 25 \mathrm{sl}$.

CO $25 ; 20 \mathrm{sl}$.

Gunnison County. USBM, DH 3-4. Minnesota Creek area, about $6 \mathrm{mi} \mathrm{E}$ of Paonia $(1,290 \mathrm{ft} \mathrm{S}$. and $1,667 \mathrm{ft} \mathrm{E}$. of the NW. cor. sec. 4, T. 14 S., R. 90 W. See USBM Bull. 550, 1956.)

CO 26; 6 sl.

CO $27 ; 22$ sl.

\#4 Coal. King mine, $6 \mathrm{mi} \mathrm{E}$ of Paonia, Delta County. (Coll. by W. L. Crentz, and A. L. Bailey, 7/16/48. See USBM Bull. 550, 1956.)

CO 28; 24 sl.

CO $29 ; 19$ sl.

Gunnison County. USBM, DH 4-10. Minnesota Creek area, about $6 \mathrm{mi} \mathrm{E}$. of Paonia. ( $700 \mathrm{ft} \mathrm{S}$. and $1,400 \mathrm{ft} \mathrm{W}$. of the NE. cor. sec. 10, T. 14 S., R. 90 W., from J. J. Dowd, 7/12/44. See USBM TP $721,1949$. 
Colorado-Continued

CO 30; 25 sl.

CO 31; 26 sl.

CO 32; 25 sl.

CO 33; 25 sl.

CO $34 ; 25$ sl.

CO $35 ; 25 \mathrm{sl}$.

CO $36 ; 10 \mathrm{sl}$.

CO $37 ; 25 \mathrm{sl}$.

CO $38 ; 18 \mathrm{sl}$.

CO 39; 25 sl.

CO 40; $15 \mathrm{sl}$.

CO 41; 15 sl.

CO 42; 22 sl.

CO $43 ; 6$ sl.

CO 44; 25 sl.

CO 45; 24 sl.

CO 46; 25 sl.

CO $47 ; 25$ sl.

CO 48; $25 \mathrm{sl}$.

CO 49; 24 sl.

CO $50 ; 25 \mathrm{sl}$.

CO $51 ; 25 \mathrm{sl}$.

CO $52 ; 23 \mathrm{sl}$.

CO 53; $16 \mathrm{sl}$.

CO 54; 25 sl.

CO $55 ; 17 \mathrm{sl}$.

CO $56 ; 15$ sl.
CO $57 ; 15 \mathrm{sl}$.

Gunnison County. USBM, DH 5-33. Minnesota Creek area, about $6 \mathrm{mi}$. E. of Paonia. ( $650 \mathrm{ft} \mathrm{N}$. and $1,035 \mathrm{ft} \mathrm{E}$. of the $S W$. cor. sec. 33, T. 13 S., R. 90 W. See USBM TP 721, 1949.)

Gunnison County. USBM DH 6-16. Coal Creek District, about 14 mi ENE. of Paonia. (600 ft S. and $100 \mathrm{ft} W$. of $\mathrm{N}^{1 / 4}$ cor. sec. 16 , T. 13 S., R. 89 W., from J. J. Dowd and J. M. S., 9/19/44.)

Gunnison County. USBM DH 13-11 and DH 14-15. Coal Creek district, about $14 \mathrm{mi}$ ENE. of Paonia. (2 sl., hole 13-11. 1,700 ft S. and $600 \mathrm{ft} \mathrm{E}$. of the NW. cor. sec. 11, T. 135, R. $89 \mathrm{~W}$.; 4 sl., hole $14-15,3,850 \mathrm{ft} \mathrm{S}$. and $1,620 \mathrm{ft}$ E. of the NW. cor. sec. 15, T. $13 \mathrm{~S}$., R. 89 W.)

Gunnison County. USBM DH 26-23. Coal Creek district, about 14 mi ENE. of Paonia. $(1,200 \mathrm{ft} \mathrm{W}$. and 2,100 ft S. of NE. cor. sec. 23, T. 13 S., R. 89 W. Sectioned by A. L. Furno and H. J. O'Donnell, analysis by $\mathrm{H}$. J. O'Donnell, Feb. 1949. Logged 1,433 ft 1 in. to $1,446 \mathrm{ft} 3$ in. See USBM Bull. 501, 1952.)

Jefferson County. Leyden hole \#l, near Golden.

Jefferson County. Leyden hole \#2, near Golden.

Jefferson County. Leyden hole \#3, near Golden.

Las Animas County. Frederick mine, Frederick \& Raton Coal Co., Valdez. (40- to 60 -in. bed thickness from J. M. S. USBM, July 1945, 8 sections from lumps.)

Las Animas County. Morley mine, Morley-Vermejo Coal Co., Morely. (66 in. thick, 7 sl.)

CO 58; $25 \mathrm{sl}$.

CO $59 ; 18 \mathrm{sl}$.

Moffat County. Knez mine, $7 \mathrm{mi}$ SE. of Craig.

CO $60 ; 25 \mathrm{sl}$.

CO $61 ; 22 \mathrm{sl}$.

CO 62; 9 sl.

CO $63 ; 25 \mathrm{sl}$.

CO $64 ; 25 \mathrm{sl}$.

CO $65 ; 25 \mathrm{sl}$.

CO $66 ; 25 \mathrm{sl}$.

CO $67 ; 10 \mathrm{sl}$.
Moffat County. Wise Hill \#2 mine, $10 \mathrm{mi}$ SW. of Craig.

Putkin County. Keenan mine (Colorado coking coal). Column sample from W. S. Landers, May 1940. See USBM Bull. 550, 1956.)

Weld County. Eagle mine, $4 \mathrm{mi} \mathrm{E}$. of Erie (E. C. E. exchange sample, June 1953. Sections from lump specimens.)
Weld County. Eagle mine, $4 \mathrm{mi} \mathrm{E}$ of Erie (Hydrogenation study. 
Colorado-Continued

CO 68; 22 sl. Weld County. Puritan mine, National Fuel Co., near Dacono. (Carbonization sample from Denver, Colo., 5/6/36 and 4/1/37. Laramie Coal? See USBM TP 622, 1941; TP 642, 1942; TP 666, 1944.)

CO 69; 22 sl. Weld County. Puritan mine, National Fuel Co., near Dacono. (Hydrogenation study. See USBM TP 622, 1941; TP 642, 1942; TP 666, 1944.)

CO 70; 19 sl. Animal Carbon Coal. (Epperson \#l, Denver, 2 sl.)

Frederick Coal. Trinidad Field. (From Reynolds, March 1944. 4 sl.)

Gunnison County. USBM DH 11-9. Coal Creek district, about 14 mi ENE. of Paonia. $(1,400 \mathrm{ft} \mathrm{N}$. and $500 \mathrm{ft} \mathrm{E}$. of the $\mathrm{SW}$. cor. sec. 9, T. 13 S., R. 89 W.: 1 sl. "eye coal" and 1 sl. kaolinite facings from the eye coal at $508 \mathrm{ft}$. See USBM Bull. 501, 1952.)

La Plata County. Monarch mine, near Durango (3 sl.).

Routt County. Wadge mine, Victor-American Fuel Co., near Mt. Harris (2 sl.).

Weld County. Columbine mine, Rocky Mtn. Fuel Co., near Serene (2 sl.).

Weld County. Grant mine, Rocky Mtn. Fuel Co., 2 mi NE of Frederick (4 sl.).

\section{UTAH}

UT 1; 25 sl.

UT 2; $25 \mathrm{sl}$.

UT $3 ; 25$ sl.

UT 4; 5 sl.

UT $5 ; 25 \mathrm{sl}$.

UT 6; $3 \mathrm{sl}$.

UT $7 ; 23$ sl.

UT $8 ; 8$ sl.

UT $9 ; 8 \mathrm{sl}$.

UT $10 ; 25 \mathrm{sl}$.

UT $11 ; 12$ sl.

UT 12; 21 sl.

UT 13; $16 \mathrm{sl}$.

UT 14; 9 sl.

UT $15 ; 13$ sl.

UT 16; 25 sl.

UT 17; 25 sl.

UT 18; 25 sl.

UT 19; 25 sl.
"A" Bed Coal. Core drill, hole 1-35, on north bank of the south fork North Creek, $337 \mathrm{ft} \mathrm{N}$. and $30 \mathrm{ft} \mathrm{E}$. of the $\mathrm{W}^{1 / 1} / \mathrm{cor}$. sec. 35 , T. 14 S., R. 5 E., Sanpete County. (Coll. by L. A. Turnbull, 3/10/42. See USBM War Minerals Report 151, March 1943; Bull. 550, 1956.)

"B" Bed Coal. Core drill, hole 1-35. As above, Sanpete County.

“C” Bed Coal. Core drill, hole 1-35. As above, Sanpete County.

"D" Bed Coal. Core drill, hole 1-35. As above, Sanpete County.

“D” Bed Coal. \#2 mine near Castlegate, Carbon County.

"E” Bed Coal. Core drill, hole 1-35. As above, Sanpete County.

Hiawatha Coal. King mine $1 \mathrm{mi}$ S. of Hiawatha, Carbon County. (Coll. by U.S. Fuel Co., 2/20/39. See USBM TP 642, 1942.)

Hiawatha Coal. King mine, $1 \mathrm{mi}$ S. of Hiawatha, Carbon County. (Cretaceous cannel coal, 5 sl. See USBM TP 642, 1942; Bull. 550, 1956.)

Sanpete County. Crystal Butte Mine, Sterling. (Horizontal section, vertical section, resin blebs, $4 \mathrm{sl}$.)

Spring Canyon \# 1 Coal. \#2 West mine, near Spring Canyon, Carbon County. (Coll. by D. J. Parker and W. W. Kessler, 7/21/39. See USBM TP 642, 1942.)

Sunnyside Coal (lower). Geneva (Horse Canyon) mine, Geneva Steel Co., Horse Canyon, $14 \mathrm{mi} \mathrm{N}$. of Woodside, and $8 \mathrm{mi} \mathrm{NE}$. of Verde, Emery County (SW/4NW// sec. 3, T. 16 S., R. 14 E. Sample from H. C. Fowler, 1/16/42. See USBM Bull. 550, 1956.) 
Utah-Continued

UT 20; 25 sl.

UT 2l; $25 \mathrm{sl}$.

UT 22; $25 \mathrm{sl}$.

UT 23; $25 \mathrm{sl}$.

UT 24; $24 \mathrm{sl}$.

UT 25; $23 \mathrm{sl}$.

UT 26; $25 \mathrm{sl}$.

UT 27; $25 \mathrm{sl}$.

UT 28; $23 \mathrm{sl}$.

UT 29; 23 sl.

UT 30; $25 \mathrm{sl}$.

UT 31; 9 sl.

UT 32; $15 \mathrm{sl}$.

UT $33 ; 13$ sl.

UT $34 ; \quad 7 \mathrm{sl}$.

UT 35; $20 \mathrm{sl}$.

UT $36 ; 3$ sl.

NM 1; 25 sl.

NM 2; $25 \mathrm{sl}$.

NM 3; 26 sl.

NM 4; 25 sl.

NM 5; 25 sl.

NM 6; 23 sl.

NM $7 ; \quad 9$ sl.

NM 8; 25 sl.

NM 9; $13 \mathrm{sl}$

NM 10; 25 sl. NM 11; 25 sl. NM 12; 12 sl.

NM 13; 13 sl.

NM 14; 13 sl.

NM 15; 25 sl. NM 16; 25 sl. NM 17; 25 sl. NM 18; 6 sl. NM 19; $12 \mathrm{sl}$.
Sunnyside coal (lower)-Continued

Lower Sunnyside Coal. Columbia mine, $3 / 4 \mathrm{mi}$ E. of Columbia, Carbon County. (Coll. by D. J. Parker and W. W. Kessler, 3/15/32. See USBM TP 573, 1937; Bull. 550, 1956.)

Wasatch Coal. Webber mine, $2 \mathrm{mi}$ E. of Coalville, Summit County. (Coll. by D. J. Parker, 8/4/39. See USBM TP 642, 1942.)

Cannel coal from $32 \mathrm{mi} \mathrm{W}$. of Salina, Sevier County.

San Rafael Swell. Emery County. (Irving Breger's Temple Mountain samples, thin sections and polished blocks.)

Lucky Strike \#2 mine, Colorado Plateau. (Coalified wood sample AW-61-54 from Irving Breger. 3 thin and 1 polished section, photo.)

\section{NEW MEXICO}

\# 31/2 Coal. Navajo \#5 mine at Gamerco, McKinley County. (Column sample from L. L. Hirst, 9/14/39. See USBM Bull. 550, 1956.)

\#5 Coal. Navajo \#5 mine at Gamerco, McKinley County. (Hydrogenation study. Column sample from L. L. Hirst, 9/14/39. See USBM Bull. 550, 1956.)

Cook \& White(?) Coal. Madrid, Sante Fe County. (4 sl., old air-shaft opening; 5 sl., northern opening, probably Miller Gulch coal.)

Cook \& White Coal. Jones mine, 1 mi N. of Madrid, Santa Fe County. (Hydrogenation study. Column sample from L. L. Hirst, 9/12/39. See USBM Bull. 550, 1956; RI 4814, 1951.)

Cook \& White Coal. Lamb mine, $1 / 2 \mathrm{mi}$. S. of Madrid, Santa Fe County. (Column sample from L. L. Hirst, 9/12/39. See USBM Bull. 550, 1956; RI 4814, 1951.)

Miller Gulch(?) Coal. USBM, DH 1-24 at $665 \mathrm{ft}$ and DH 3-24 at $304 \mathrm{ft}$ to $382 \mathrm{ft}$, Madrid, Santa Fe County. (Samples from A. L. Toenges, Feb. and Mar. 1943.)

Miller-Gulch Coal. Santa Fe County outcrop. (Sections from A. L. Toenges, USBM, Mar. 1943.)

Raton Coal. Dawson \#6 mine, 11/2 mi NE. of Dawson, Colfax County. (Column sample from L. L. Hirst, 9/11/39. See USBM Bull. 550, 1956.)

Socorro County. Carthage. (Sample A-158 from A. L. Toenges, USBM, Mar. 1943.) 


\section{TEXAS}

TX 1; 6 sl. Dimmit County. Doloris Field. (Grab samples from W. A. Selvig, Mar. 1943, 4 sl.)

Dimmit County. Southeast Dimmit County. (Sec. 30, oil exploration sample from ll-ft thickness of uncharted coal field, from D. W. Spitzer, Acreage Dept., A. F. Wilcox Oil \& Gas Co., Tulsa, Oklahoma, Sept. 1937. 2 sl.)

TX 2; 25 sl.

Freestone County. Core hole, Texas Power Co., $3.7 \mathrm{mi}$ NNW. of

TX 3; $20 \mathrm{sl}$.

Fairfield. (Sample from V. F. Parry, USBM. See USBM Bull. 550, 1956.)

TX 4; 26 sl.

Grimes County. \# 1 core hole, $3 \mathrm{mi}$ S. of Bedia. (Lignite from V.

TX $5 ; 25 \mathrm{sl}$. F. Parry, USBM.)

TX 6; $25 \mathrm{sl}$.

TX $7 ; 25$ sl.

TX $8 ; 19$ sl.

TX 9; 25 sl.

Harrison County. Darco mine, $12 \mathrm{mi}$ SW. of Marshall. (Lignite column sample of upper and lower benches from B. C. Parks, USBM, and H. J. O'Donnell, USBM, Sept. 1947. See USBM Bull. 550, 1956; RI 482, 1950.)

TX 10; 15 sl. Henderson County. (Lignite from Malekoff Fuel Co., 9/28/34.)

TX 11; 10 sl. Milam County. Sandow mine, near Sandow. (Lump specimens June 1953.)

TX 12; 14 sl. Wise County. Bridgeport. (C. B. Singleton Coal Co. mine, 7 sl.; Byrns \& Byrns Coal Co. mine, 7 sl.; from W. A. Selvig, 11/27/40. Collected by Kintz, Hynal, Hanahen, Dallas, Tex.)

TX 13; 23 sl. Wood County. \#16 mine, Consumers Lignite Co., near Alba. (Hydrogenation study. Sent $1 / 6 / 39$. See USBM TP 642, 1942.)

TX 14; 3 sl. Freestone County. Big Brown mine, (41A area), N. of Fairfield. (Eocene Coal sample collected by J. M. S., USGS, 11/15/73. See G.S.A. Fieldtrip guidebook, Kaiser \& Groat, Nov. 1973.)

\section{NEW JERSEY}

NJ 1; 1 sl. Lignitic wood. E. C. E. exchange sample. Monmouth County Squire Signal Lab., Fort Monmouth, near Eatontown.

\section{UNIT 5. WEST COAST STATES AND ALASKA WASHINGTON}

WA $1 ; 23$ sl. WA $2 ; 7 \mathrm{sl}$.

\#1 Coal. Reliance \#2 mine, 21/2 mi E. of Chehalis, Lewis County. (Hydrogenation study. Lignite from H. F. Yancey, Feb. 1939. See USBM TP 642, 1942.)

WA 3; $10 \mathrm{sl}$. \#1 Coal. Glen Echo mine, $10 \mathrm{mi}$ NE. of Bellingham, Whatcom County. (Hydrogenation study. Sample from H. F. Yancey, USBM, Sept. 1938.)

WA 4; 8 sl. \#l Coal and \# 2 Coal. Tiger Mountain \#l mine, about $5 \mathrm{mi}$ SE. of Issaquah, King County (woody coal).

WA $5 ; 25$ sl. WA $6 ; 25 \mathrm{sl}$. \# 2 Coal. Bartoy mine, near Wilkeson, Pierce County. (Sample from WA $7 ; 22 \mathrm{sl}$. 
Washington-Continued

WA 8; 25 sl. \#5 (Miller) Coal. Wilkeson-Miller mine, near Wilkeson, Pierce WA 9; 25 sl. County. (Sample from M. R. Geer and A. Centenero, 6/20/40. See WA $10 ; 6 \mathrm{sl}$. USBM TP 649, 1942.)

WA 11; 21 sl. \#9 Coal. Grand Ridge mine, near Grand Ridge, 3 mi NE. of Issaquah, King County. (Sample from H. F. Yancey, Aug. 1938. Hydrogenation study. See USBM TP 642, 1942.)

WA 12; 5 sl. Big Dirty Coal. Kostic Prospect outcrop, Lewis County. (NW/1/4NE sec. 16, T. 14 N., R. 1 W. Snavely CC-5. See USGS Bull. 1053, 1958.)

WA 13; 7 sl. Black Bear Coal. Black Jewell mine, Thurston County. (NW/1/4NW1/4, sec. 32, T. 16 N., R. 1 W. Snavely CC-1. See USGS Bull. 1053, 1958.)

WA 14; 6 sl. Little Dirty Coal. Holiday Road outcrop, Lewis County (NW1/4SW1/4 sec. 35 , T. 15 N., R. 2 W. Snavely CC-4. See USGS Bull. 1053, 1958.)

WA 15; 25 sl. McKay Coal. Strain-Upper Diamond mine, Strain Coal Co., 1/1/2 mi WA 16; 9 sl. NE. of Black Diamond, King County (Hydrogenation study. Sample from Dr. Storch, Renton, Wash. Feb. 1939. See USBM TP 622, 1941; TP 642, 1942; TP 666, 1944.)

WA 17; 8 sl. Mendota Coal. Boxer mine, Thurston County. (Sec. 14, T. 51 N., R. 2 W. 3 sl.)

Mendota Coal. Columbia Coal Co., Lewis County. (From H. F. Yancey, USBM, 4/5/44. 5 sl.)

Miller Coal or \#5. See \#5 above.

WA 18; 5 sl. $\quad$ Smith Coal. Belle Slope mine, Lewis County. (NE $1 / 4 \mathrm{SW}^{1 / 4}$ sec. 34, T. 35 N., R. 1 W. Snavely CC-3. See USGS' Bull. 1053, 1958.)

WA 19; 5 sl. Upper Thompson Coal. Stoker mine, Lewis County (NE/4 SW/1/4 sec. 29, T. 15 N., R. 2 W., Snavely CC-6, 4 sl. See USGS Bull. 1053, 1958.)

Upper Thompson Coal Bed. Centralia Field? (1 sl.).

WA 20; 10 sl. Tono \#l Coal. Tono strip pit, Thurston County (NW1/4/2W/4 sec. 21, T. 15 N., R. 1 W.; 4 sl., lower bench Snavely CC-7, 6 sl., top bench Snavely CC-8. See USGS Bull. 1053, 1958.)

WA 21; 17 sl. Cowlitz County. Silver Lake mine, about $4 \mathrm{mi} \mathrm{NE}$. of Silver Lake, about $1 \mathrm{mi} \mathrm{W}$. of Toutle River fork. (RT-52-9, 6 sl.; surface outcrop, PO-52-3, 11 sl.)

WA 22; 16 sl. Cowlitz and Lewis Counties. USBM clay pit \#1, Cowlitz County (9 sl.); Cedar Creek Falls, Lewis County about $6 \mathrm{mi} \mathrm{SE}$. of Toledo. (RT-5l-6, 7 sl.)

WA 23; 17 sl. Cowlitz and Lewis Counties. Leavell mine, about 2 mi ESE. of Castle Rock, Cowlitz County. (4 sl. See USGS Bull. 1062, 1958.)

Schuff mine. About $2 \mathrm{mi}$ N. of Castle Rock, Cowlitz County (4 sl.).

Walker mine, About $3 \mathrm{mi} \mathrm{NW}$. of the Toutle River fork, Cowlitz County, about $1 / 2 \mathrm{mi} \mathrm{E}$. of the river $(8 \mathrm{sl}$.).

Creek outcrop. Smokey Valley prospect, Lewis County. (SW/4NW1/4 SW $1 / 4$ sec. 31, T. 11 N., R. 1 W. 1 sl.)

WA 24; 23 sl. Cowlitz \& Lewis Counties. Idleman mine, Cowlitz County, about $1 / 2$ mi NE. of Castle Rock. (Shives \#1 \& \#.2 Pits 9 sl.) Lewis County about $8 \mathrm{mi}$ E. of Toledo, near Windom. (Pit \#1 - 6 sl.; Pit \#2 8 sl.) 
Washington-Continued

WA 25; 10 sl. Jefferson County. Creek at Ocean Beach. (NE ${ }^{1 / 4 \mathrm{SE}^{1 / 4}}$ sec. 17, T. 25 N., R. 13 W. Pleistocene-brown coal.)

WA 26; 6 sl. King County. Taylor mine, Nat. Develop. Co., near Taylor. (Hydrogenation study. Sample from H. F. Yancey, Sept. 1938. See USBM TP 642, 1942.)

WA 27; 24 sl. King County. Prospect drift opening, Youngblood Farm, 7 mi E. of Auburn. (Hydrogenation study. Pleistocene brown coal sample from H. F. Yancey, See USBM TP 642, 1942; Bull. 550, 1956.)

WA 28; 3 sl. Lewis \& Cowlitz Counties. (Hydrogenation study. Brown coal sample from H. F. Yancey, USBM, Nov. 1938. Collected by R. W. Fletcher of Winlock, Wash.)

WA $29 ; 10$ sl. Lewis County. Monarch \#2 mine, $7 \mathrm{mi} \mathrm{E}$. of Centralia.

WA 30; 24 sl. Lewis County. USBM, DH 1-15, about $8 \mathrm{mi} \mathrm{E}$. of Toledo. (92 ft N., $30 \mathrm{ft} \mathrm{E.} \mathrm{of} \mathrm{SW} 1 / 4$ cor. sec. 15, T. 11 N., R. 1 E. Cored 5/28-6/5/45.)

WA 31; $14 \mathrm{sl} \quad$ Lewis County. USBM diamond drill cores, about $8 \mathrm{mi}$ E. of Toledo. (T. 11 N., R. 1 E.)

DH 2-15; $118 \mathrm{ft} \mathrm{N}$., $470 \mathrm{ft}$ E. of $\mathrm{S}^{1 / 4}$ cor. sec. 15. (Cored 6/7-13/45. 5 sl.)

DH 8-15; $995 \mathrm{ft}$. N., $1330 \mathrm{ft}$ W. of $S^{1 / 4}$ cor. sec. 15. (Cored 7/9-11/45. 3 sl.)

DH 11-15; $90 \mathrm{ft} \mathrm{N.} 915 \mathrm{ft} \mathrm{W}$. of $\mathrm{S}^{1 / 4}$ cor. sec. 15. (Cored 7/24-27/45. 5 sl.)

DH 28-15; $525 \mathrm{ft}$ N., $835 \mathrm{ft}$ E. of SW. cor. sec. 15. (Cored 9/28-10/1/45. 1 sl.)

WA 32; 18 sl. Lewis County. As above.

DH 9-22; 825 ft S., 510 ft E. of N1/4 cor. sec. 22. (Cored 7/12-16/45. 7 sl.)

DH 15-22; $770 \mathrm{ft} \mathrm{S.,} 400 \mathrm{ft}$ W. of N//4 cor. sec. 22. (Cored 8/10-14/45. 8 sl.)

DH 32-22; 1,365 ft S., $270 \mathrm{ft}$ E. of N1/4 cor. sec. 22. (Cored 10/20-24/45. 3 sl.)

WA 33; $8 \mathrm{sl}$. Lewis County. Black Prince mine, $7 \mathrm{mi} \mathrm{NE}$. of Centralia, $1 / 4 \mathrm{mi} \mathrm{E}$.

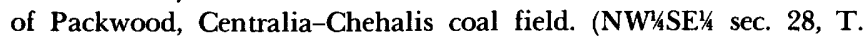
15 N., R. 1 W. Vitrain $\log$ from Tono \#1 coal.)

WA 34; 18 sl. Lewis County. Centralia-Chehalis district. Tono cores.

Hole "DD"; NE $1 / 4$ SW1/4 sec. 29, T. 15 N., R. 2 W. (8 sl.)

Hole "V"; NE $1 / 4 \mathrm{NE}^{1 / 4}$ sec. 33, T. 14 N., R. 2 W. (2 sl.).

Hole "XX" (6 sl.).

Hole "VV" (2 sl.).

WA 35; 3 sl. Miocene Coal. (Sample from R. D. Brown, 1955.)

\section{OREGON}

OR 1; 7 sl. Newport (Beaver Hill) Coal. Coos Bay coal field. ${ }^{2}$ Southport mine, about $4 \mathrm{mi} \mathrm{S.} \mathrm{of} \mathrm{Marshfield,} \mathrm{Coos} \mathrm{County.} \mathrm{(Sample} \mathrm{from} \mathrm{J.} \mathrm{J.} \mathrm{Dowd,}$ Feb. 1944.)

${ }^{2}$ See USBM TP 707 (1948) for detailed information on items from the Coos Bay coal field. 
Oregon-Continued

OR 2; 21 sl. Newport (Beaver Hill) Coal. Coos Bay coal field. ${ }^{2}$ Thomas mine, about $10 \mathrm{mi}$ S. of Marshfield, Coos County. (Hydrogenation study. Sample from M. R. Geer, 9/25/39. See USBM TP 642, 1942; Bull. 550, 1956.)

OR 3; 14 sl. Coos County. Coos Bay coal field. ${ }^{2}$ USBM, DH 12-16 about $1 / 2 \mathrm{mi}$ W. of Coaledo.

OR 4; 14 sl. Coos County. Coos Bay coal field. ${ }^{2}$ USBM, DH 15-3 about $2 \mathrm{mi}$ N. of Coaledo.

OR 5; 13 sl. Coos County. Coos Bay coal field. ${ }^{2}$ USBM DH 9-15, about 1/4mi ENE. of Coaledo.

OR 6; 9 sl. Coos County. Coos Bay coal field. ${ }^{2}$ USBM DH 13-16, about $1 \mathrm{mi}$ OR $7 ; 21$ sl. W. of Coaledo.

OR 8; 15 sl. Coos County. Coos Bay coal field. ${ }^{2}$ USBM drill cores.

DH 6-3, about $2 \mathrm{mi} \mathrm{N}$. of Coaledo (3 sl.).

DH 7-10, about $1 / 1 / 4 \mathrm{mi} \mathrm{NE}$. of Coaledo (1 sl.).

DH 8-3, about 1/1/4 mi NNE. of Coaledo (1 sl.).

DH 10-15, SE. cor. of Coaledo (3 sl.).

DH 11-10, about $1 \mathrm{mi} \mathrm{N}$. of Coaledo (3 sl.).

DH 14-3, about 1 1/2 mi NNE. of Coaledo (3 sl.).

Coos Bay. Residue A-49.1 (1 sl.).

OR 9; 2 sl. Coos County. Coos Bay coal field. ${ }^{2}$ Overland mine, about 1 mi NW. of Coaledo.

\section{CALIFORNIA}

CA 1; 10 sl. Amador County. Edwin \#2 claypit, near Ione. (Lignite, 4 sl. from selected lumps; 2 sl., residue from extraction P-12-Z 49.2; 1 sl., untreated residue from sample 45736; 2 sl., anthraxylon after extraction 45737; 1 sl., from sample 45474.)

CA 2; 25 sl. Amador County. Ione mine, American Lignite Products Co., $1 \mathrm{mi}$ CA 3; 25 sl. SW. of Ione. (Column sample from T. R. Jolley and A. L. Toenges, CA 4; 25 sl. 2/10/49, sectioned A. L. Furno, and petrographic analysis by $H$. CA 5; 23 sl. J. O'Donnell, completed June, 1949. See USBM Bull. 550, 1956.)

\section{NEVADA}

NV 1; 7 sl. Diamond Peak Coal. Cherry Creek Mountains, White Pine County (2 sl.).

Esmeralda County. Near Coaldale, slope $5 .(2,300 \mathrm{ft}$ N. and $400 \mathrm{ft}$ W. of the SE. cor. sec. 29, T. 2 N., R. 37 E., Mount Diablo Meridian. Bench 3, 1 sl.; bench 6, 2 sl.; bench 8, 1 sl.; 2 polished blocks from bench 6; 1 sl., igneous rock. See USBM TP 687, 1946.)

NF 2; 18 sl. Esmeralda County. Near Coaldale. (See USBM TP 687, 1946.)

USBM, DH 1-28 (SW1/4W1/4NE $1 / 4 \mathrm{SW}^{1 / 1} / 4$ sec. 28, T. 2 N., R. 37 E., 668 ft $1 / 2$ in. to $671 \mathrm{ft} 3 \frac{1}{2}$ in., 12 sl.)

USBM, DH 2-33 (SW1/4 $\mathrm{NE}^{1 / 4} \mathrm{NE}^{1 / 4}$ sec. 33, T. 2 S., R. 37 E., $359 \mathrm{ft}$ 2 in. to $360 \mathrm{ft} 2$ in., $3 \mathrm{sl}$.)

USBM, DH 3-28 (SW'1/4NE $1 / 4 \mathrm{SW}^{1 / 4} \mathrm{NW}^{1 / 4} / 4$ sec. 28, T. 2 S., R. 37 E., 384 ft to $385 \mathrm{ft}, 3 \mathrm{sl}$.)

2See USBM TP 707 (1948) for detailed information on items from the Coos Bay coal field. 


\section{ALASKA}

AK 1; 25 sl. AK 2; 25 sl. AK $3 ; 25$ sl. AK $4 ; 24$ sl.

AK $5 ; 25$ sl. AK 6; 25 sl. AK $7 ; 25$ sl. AK $8 ; 18$ sl.

AK 9; 25 sl. AK 10; 25 sl. AK 11; 25 sl. AK 12; 5 sl.

AK 13; 24 sl. AK 14; 25 sl. AK 15; 24 sl. AK 16; 25 sl.

AK 17; 25 sl.

AK 18; 25 sl.

AK 19; 25 sl.

AK 20; 25 sl.

AK 2l; 4 sl.

AK 22; 4 sl.

ANT 1; 1 sl. AST 1; 12 sl.
\#3 Coal. Evan Jones mine, at Jonesville, Matanuska Field (Bottom 76 in. column from T. R. Jolley, 10/11/49. See USBM Bull. 550, 1956.)

\#4 Coal. Suntrana mine at Suntrana, Nenana district. (Hydrogenation study. Column sample from M. L. Sharp, Anchorage, Alaska 6/20/40. Includes 3 misc. slides from Reynolds, Nov. 1943. See USBM Bull. 550, 1956.)

\# 8 Coal. Evan Jones mine at Jonesville, Matanuska Field. (Hydrogenation study. Column sample from Maurice L. Sharp, 8/3/40. See USBM Bull. 550, 1956.)

Donaldson \# 3 Coal. Suntrana mine at Suntrana, Nenana district. (Column sample from M. L. Sharp, June 1942. See USBM Bull. $550,1956$.

Upper Shaw Coal. Eska mine, Eska, Lower Matanuska Field. (Hydrogenation study. Column sample from M. L. Sharp, $8 / 5 / 40$. See USBM Bull. 550, 1956.)

Off Amchitka Island. Coalified wood from the Bering Sea, off Amchitka Island. (Coal Geology Laboratory, Columbus, Ohio. 3 sl. +2 blocks.)

Roth Prospect Mine. $8 \mathrm{mi}$ E. of Suntrana (Subbituminous, subcannel coal, sclerotia, and other fungal remains. 1 sl.)

\section{UNIT 6. FOREIGN AREAS}

\section{ANTARCTICA}

Coal collected 10,000 ft above sea level near the South Pole by Admiral Byrd, 1937. (Corresponding block of coal included.)

\section{AUSTRALIA}

New South Wales. Bulli Coal. Illaware coal field, Burragorang Valley. (Coal block specimen \#4, Wollondilly extended colliery from Sydney (Australia) County Council, fall 1944. 2 sl.)

Greta Main (Upper) Coal. Maitland coal field, Millifield colliery. (Coal block specimen \#5. 2 sl.)

Greta (Lower) Coal. Maitland coal field, Ayrfield \#3 colliery near Branxton. (Coal block specimen $\# 2$ from Sydney (Australia) County Council, fall 1944. 2 sl.)

Lithgow Coal. Western coal field, Lithgow Valley colliery. (Coal bed specimen \#3. 4 sl.)

Wallarah Coal. Northern (New Castle) coal field, north Wallarah colliery. (Coal block specimen \#1. 2 sl.) 
Australia-Continued

AST 2; 20 sl. Rosewood District, Hoskisson Upper Coal. Gunnedah (6 sl.).

Melville Lower Coal. (3 sl.).

Lanefield Cannel Coal. Specimen B (6 sl.).

Lanefield Bright Coal. May 1951. Specimen A (5 sl.).

AST 3; 19 sl. Victoria, Yallourn Brown Coal.

\section{BELGIUM}

BLG 1; 19 sl. Lignite. E. C. E. exchange sample. June 1953, Florennes.

\section{BRAZIL}

BRA 1; 17 sl. Rio Negro Coal. Upper and lower benches, State of Rio Grande Do Sul. (Column sample from Thomas Fraser, 1944.)

BRA 2; 15 sl. Barro Branco Coal. Upper and lower benches, Bainho mine, State of Santa Catarina. (Sample from Thomas Fraser 1944. CG1 Mac. 634, trilete spores.)

\section{CANADA}

CAN 1; 10 sl.

Alberta. Mine \#3-\#.434-33 Lethbridges (2 sl.).

Mine \# 771-\# 424-32 Coalspur area, foothills (3 sl.).

Mine \#846-\#309-33 Coalspur area, Mercoal (2 sl.).

Mine \#852-\#302-31 Saunders area, Alexandria (3 sl.).

CAN 2; 10 sl. Alberta. Subbituminous E. C. E. exchange sample. Mar. 1955.

CAN 3; 16 sl. Nova Scotia. Harbour Coal, from Dominion Iron and Steel Co., Oct. 1939.

CAN 4; 24 sl. Nova Scotia. Blockhouse Coal, from Dominion Iron and Steel Co., Oct. 1939.

CAN 5; 5 sl. Nova Scotia. Phelan Coal, Sydney (2 sl.).

Ontario. Onakawana lignite (1 sl.).

British Colunbia. E.C.E. exchange sample (2 sl.).

\section{CHILE}

CHL 1; 25 sl.

Mina Elena. On Riesco Island, Magallanes Province (lignite).

CHL 2; 16 sl.

CHL 3; 20 sl.

Pupunahue Mine, about $2.5 \mathrm{mi} \mathrm{SE}$. of Mulpun and about $28 \mathrm{mi}$ ENE. of Valdivia (subbituminous).

CHL 4; 25 sl. Vulcano Mine, $14 \mathrm{mi} \mathrm{NW}$. of Punta Arenas, Magallanes Province (lignite).

\section{CHINA}

CHN 1; 5 sl. Bony Coal. Feng mine, Tien Fu Mining Co., Ltd., 18 km SW. of Teipay, Szechuan, China (Chunking Prefecture, 4/11/45. 3 sl. bony and 2 sl. gocd coal.) 


\section{COLOMBIA}

COL 1; 12 sl. El Placer Coal. St. Vincente mine, near Sesquille (State or county) Cundinamarcá ( 1 sl.).

Grande Laureles \& Canal Coal. Rodomontal mine, Cogue. (M-1 Paba. 2 sl.)

LaVirginia Coal. St. Vincente mine, town of Sesquille (State or county) Cundinamarca. (M-3 Paba. 1 sl.)

Canoas Mine. Soacha. (M-7 Paba. 2 sl.)

Cincha Mine, Near Suesca (State or county) Cundinamarca. (M-6 Paba. 1 sl.)

Cogonta Mine, Suesca. (M-5 Paba. 2 sl.)

El Desague Mine. Verganzo Hacienda, near Tocancinda, (State or county) Cundinamarca. (M-2 Paba. 1 sl.)

La Espernanza Mine. Choconta, seams 1, 2, 3 \& 4(?). (M-4 Paba. 2 sl.)

Rodomontal Mine. Cogue. Grande Laureles, cannel coal (2 sl.).

St. Vincente Mine. Alfonso-Rodriguez working, near Sesquille (State or county) Cundinamarca. (M-3 Paba. LaVirginia coal, 1 sl.; El Placer coal, 1 sl.)

\section{DENMARK}

DEN 1; 3 sl. Mine in the Faeroe Islands. From Newell S. Alford to Mr. Greenwald, 8/11/38.

\section{ENGLAND}

ENG 1; 16 sl. 'Barusby (Barnsely?) Coal. (1 sl.).

Beeston Coal. (8 sl.).

"Silkstone". (1 box, 7 sections + 5 lumps.)

ENG 2; 9 sl. Ravine Mine. Lancashire coal field (6 sl.).

Nottinghamshire, Durham, and Northumberland.

1) Section H. Canneloid material, deep hard seam, Nottinghamshire (1 sl. boghead, 1 lump).

2) Section J. Canneloid material, Bookwell seam, Durham (1 sl. cannel, 1 lump).

3) Section K. Hards, Pléssey seam, Northumberland. (1 sl. + 1 lump from Miss Stadnichenko, 3/31/36.)

\section{GERMANY}

GER 1; 6 sl. Alesander Schaute Mine. From Hoffman (incl. lumps).

GER 2; 7 sl. $\quad$ Bjufs Mine. (1 sl.).

Brassert Mine. Bright and splint from Hoffman (incl. 10 lumps, $6 \mathrm{sl}$.).

GER 3; 7 sl. Piessenberg Mine. From Hoffman (includes lumps).

GER 4; 10 sl. Rieback Mine. Brown coal.

GER 5; 22 sl. Ruhr Coal. 


\section{EAST GERMANY (D.D.R.)}

GER 6; 10 sl. Region of Aschersleben (D.D.R.). Eocene brown coal. (E.C.E. exchange sample, June 1954.)

GER 7; 20 sl. Region of Bitterfeld (D.D.R.). Oligocene brown coal. (E.C.E. exchange sample, June 1954. 10 sl.)

Region of Borna (D.D.R.). Eocene brown coal. (E.C.E. exchange sample, June 1954. 10 sl.)

GER 8; 19 sl. Region of Geiseltal (D.D.R.) SW. of Halle (a.S.) Eocene brown coal. (E.C.E. exchange sample, June 1954. 9 sl.)

Region of Halle (a.S.) (D.D.R.). Eocene brown coal. (E.C.E. exchange sample, June 1954. $10 \mathrm{sl}$.)

GER 9; 19 sl. Region of Niederlausitz (D.D.R.). Miocene. brown coal. (E.C.E. exchange sample, June 1954.)

GER 10; 16 sl. Region of Oberlausitz (D.D.R.). Miocene brown coal. (E.C.E. exchange sample, June 1954. 10 sl.)

Region of von Bautzen (D.D.R.). Miocene brown coal. (E.C.E. exchange sample, June 1954. 6 sl.)

\section{WEST GERMANY (D.F.R)}

GER 11; 10 sl. (D.F.R.) Brown coal. (E.C.E. exchange sample, June 1953. Rheinische Rohbraunkohle mine(?).)

GER 12; 6 sl. Cannel brown coal. Habirspisk. Bohemia (1 sl.).

Florentine Mine. Beuthen, Inst. of Geology and Mineralogy. Deposits, Leoben (Heinzmann flöz, 1 sl.; Sattel flöz, 1 sl.)

Polished block. Zeche Ehrenberg Fortesets. (Relief section 466. Dr. Erich Stach, Coal Petrographic Laboratory.)

Brown Coal. (E.C.E. exchange sample. 2 sl.)

E.C.E. exchange sample (1 sl.).

\section{GREECE}

GRE 1; 20 sl. Aliveri Mine. Lignite. (E.C.E. exchange sample. Island of Evvoia (Euboea), Jan. 1954.)

Peristri Mine. Lignite, Athens area. (E.C.E. exchange sample, Jan. 195410 sl.)

GRE 2; 10 sl. Ptolemais Mine. Lignite, west Macedonia. (E.C.E. exchange sample, GRE 3; 19 sl. Jan. 1954.)

GRE 4; 10 sl. Serres Mine. Lignite, eastern Macedonia. (E.C.E. exchange sample, Jan. 1954.)

GRE 5; 5 sl. Attica County. Magara. (Lignite sections (lumps) from V. F. Parry, USBM.)

GRE 6; 25 sl. Coal \#126-B.

\section{ITALY}

ITL 1; 20 sl. Toscana-Valdamo Mine. Lignite. (E.C.E. exchange sample, June 1953. 10 sl.)

Mercure Mine. Lucania. (E.C.E. exchange sample, June 1953. 10 sl.) 
Italy-Continued

ITL 2; 20 sl. Region of Toscano. Lignite. (E.C.E. exchange sample, Apr. 1954. 10 sl.)

Spoleto Mine. Umbria. (E.C.E. exchange sample, June 1953. 10 sl.)

\section{MALAYA FEDERATION}

MAY 1; 12 sl. Batu Arang. Selanzor. (E.C.E. exchange sample, Mar. 1955. 10 sl.)

Lignite Coal. (2 sl. + lumps).

MEXICO

MEX 1; 3 sl.

POLAND

POL 1; 14 sl. Brown coal. (E.C.E. exchange sample, Sept. 1953.)

\section{REPUBLIC OF SOUTH AFRICA}

SAR 1; 25 sl. Cuticle Coal. Molteno coal horizon, Umkomaas Valley, about 15 mi NW. of Himeville, Natal (1 sl.).

D.N.C. Coal. Druban Natal Colliery \#3 mine, $1 \mathrm{mi}$ S. of Dannhauser (3 sl., upper bed; 1 sl., lower bed).

Dullstroom Coal. (3 sl.).

Satmar "B" Coal. Satmar plant, about $8 \mathrm{mi} \mathrm{N}$. of Ermelo, Transvaal (8 sl.).

Witbank \#1 Coal. South Section mine, Witbank Colliery Co. (4 sl.).

Witbank \#2 Coal. As above (1 sl.).

Witbank \# 5 Coal. South African Coal Estates Co. Navigation Collịery (3 sl.).

Boghead Coal. Permian, Ludafoska Ermelo Transvaal 1/15/34 W.S.M. (1 sl.).

\section{RHODESIA}

ROD 1; 1 sl. Wanki Coal. Wanki mine (3/11/25).

\section{SWEDEN}

SWE 1; 5 sl. Mesozoic Coals. Scania, southern Sweden. Lower Rhaetic coal from Vallakra (3 sl.).

Middle Jurassic coal from Eriksdal in Fyledalen (2 sl.).

U.S.S.R.

RUS 1; 5 sl. $\quad$ Russian Sapropel.

\section{VENEZUELA}

VEN 1; 8 sl. Oficina Formation, eastern Venezuela, Venezuela Oficina Coal (N2-613, RG-65, NG-508, ZG-115, ZG-4, NS-724).

\section{YUGOSLAVIA}

YUG 1; 10 sl. Lignite, Kolubara, (Serbia). (E.C.E. exchange sample, June 1953.)

YUG 2; 18 sl. Lignite, Velenje, (Slovenia). (E.C.E. exchange sample, July 1953. 10 sl.)

Lignite, Kreka, (Bosnia). (E.C.E. exchange sample, July 1953. 8 sl.) 


\section{UNITS 7-9. SPECIAL CATEGORIES \\ 7. LOMAX, JEFFREY, F. D. R., AND R. THIESSEN EARLY STUDIES} LOMAX SECTIONS (ENGLAND, 1 SL. EACH)

Lom 1; 9 sl. $\quad$ 1) Silkstone bed, Altofts mine, Pope \& Pearson Ltd., Normanton (vertical section).

2) Silkstone bed, top part, Altofts mine, Pope \& Pearson Ltd., Normanton (horizontal section).

3) Cannel mine, top part, Maypole Colliery, Moss Hall Coal Co., Ltd., Abram (vertical section).

4) Cannel mine, top part, Maypole Colliery, Moss Hall Coal Co. Ltd., Abram (horizontal section).

5) Rams mine, Pendleton Colliery, A. Knowles, Ltd. (horizontal section).

6) Rams mine, Pendleton Colliery, A. Knowles, Ltd. (vertical section).

7) Ravin mine, Lancashire.

8) Arley mine, Lancashire.

9) Mountain mine, Lancashire.

\section{E.C. JEFFREY (CELLOIDIN TECHNIQUE) MICROTOME SLIDES}

Jef 1; $25 \mathrm{sl}$. United States.

Illinois, Gallatin County, bituminous coal, 1 horizontal section. Illinois, Gallatin County, bituminous coal, 1 vertical section. Indiana, Vigo County, bituminous coal, 1 vertical section.

Kentucky, boghead coal, contains supposed algae, 2 sections.

Kentucky, cannel coal, 1 horizontal section, 1 vertical section.

Kentucky, bituminous coal, 1 horizontal section.

Kentucky, bituminous coal, 1 vertical section.

Massachusetts, Cambridge, kettlehole $30 \mathrm{ft}$ deep, polleniferous peat, 1 section.

New York, Kreischerville, Staten Island, lignitic wood, 1 section. Anthracite(?) splint. 2 vertical sections.

Anthracite(?) cannel, $\mathrm{Pa} .2$ vertical sections.

Australia. Neunes, New South Wales, oil shale, excellent Botryococcus, 1 section.

Canada. Cape Breton, Nova Scotia, 1 vertical section.

Canada. Upper Devonian coal, 2 sections: 1 vertical, 1 horizontal.

England. Lancashire, bituminous coal, 1 section

New Zealand. Eocene age coal, 1 section.

Sweden. Upper Cambrian coal, 2 sections.

Tasmania. Tasmanite, 2 sections: 1 vertical, 1 horizontal.

F. D. R. MICROTOME SLIDES, 1930

Fdr 1; 10 sl. Upper Freeport Coal. Cannel, High Shaft mine (8 sl.)

Illinois No. 5 Coal. La Chede mine (1 sl.)

No. 6 Coal. Zeigler mine (1 sl.) 


\section{R. THIESSEN'S EARLY STUDIES}

(REPORTED IN USBM BULL. 38 (1913) AND USBM BULL. 117 (1920))

Thi $1 ; 16$ sl. Ind. \#5 Coal. Vandalia mine, Terre Haute, Vigo County, Ind. (10 thin sections).

DeWitt Lignite North Dakota (6 microtome sections).

Thi 2; 22 sl. Kiskaminetas Cannel Coal. Beaver County, Pa. (22 thin sections).

Thi 3; 23 sl. Pratt Coal. Gorgas mine, Gorgas, Walker County, Alabama (9 thin sections).

Thick Upper Freeport. New Fields mine, north Bessemer, Allegheny County, Pa. (5 thin sections).

Thick Upper Freeport. Consumers mine, Oakmont, Allegheny County, Pa. (6 thin sections).

Pittsburgh Coal. Pittsburgh, Allegheny County, Pa. (1 thin section). Upper Kittanning Coal. Paragon mine, Cannelton, Beaver County, Pa. (2 thin sections).

Thi 4; 22 sl. Sipsey Coal. Sipsey, Walker County, Ala. (5 mac. sl.).

Pittsburgh Coal. Bruceton, Allegheny County, Pa. (4 mac. sl.).

Ill. No. 6 Coal. Royalton mine, Royalton, Franklin County, Ill. (2 mac. sl.).

Ill. No. 6 Coal. Hart Williams mine, Franklin County, Ill. (1 mac. sl.).

Ill. No. 6 Coal. Zeigler mine, Zeigler, Franklin County, Ill. (9 mac. sl.).

Thi 5; 19 sl. Ill. No. 6 Coal. Sesser mine, Sesser, Franklin County, Ill. (3 mac. sl.).

Ill. No. 2 Coal. Barry coal bank, Exeter, Scott County, Ill. (6 mac. sl.).

Ill. No. 14 Coal. Shelbyville mine, Shelbyville, Shelby County, Ill. (2 mac. sl.).

Lower Freeport Coal. New Fields mine, north Bessemer, Allegheny County, Pa. (10/24/19) (mac. sl.).

Ind. No. 5 Coal. Vandalia mine, Terre Haute, Vigo County, Ind. (4 mac. sl.).

Thi 6; 22 sl. Thin sections of fossil wood, (Tertiary(?)).

Specimen 1. Silicified gymnospermous wood. Wilton, N. Dak. (6 sl.).

Specimen 2. Silicified gymnospermous wood. Ole (Cle?) Elum (5 sl.).

Specimen 3. Silicified gymnospermous wood. Ralston (2 sl.).

Specimen 4. Silicified gymnospermous wood. Locality(?) (2 sl.).

Specimen 5. Silicified angiospermous wood. Locality(?) Tyloses, pyritic ( 5 sl.).

Specimen 6. Silicified conifer wood. Albuquerque, N. Mex. (1 sl.).

Specimen 7. Silicified conifer wood. Newcastle, Wyo. (1 sl.) 


\section{OIL SHALE AND RELATED ROCKS}

\section{UNITED STATES}

Osr 1; 8 sl. Alaska.

Mineral resembling Torbanite, in boghead coal (1 sl.).

Tasmanite rock, Kuna-Ipnavik Rivers. (Pellet \#3193 of Tomich, B.C.R. Sample 64ATr 209A, 3 sl., 1 pellet.)

Tuxecan Island. $72 \mathrm{mi}$ NW. of Ketchikan. (Carbonaceous matter in limestone breccia. \#54AE31. 2 thin sections +2 residues slides + remaining breccia fragments.)

Osr 2; 2 sl. Indiana.

Black shale above Indiana IV Coal. Linton \#28 strip pit, Green County. Maumee Colliery Co. (May 1951).

Osr 3; 24 sl. Kentucky.

Bath County. Oil shale \#6, about 1/2 mi from Salt Lick on the Caney road (1 sl.).

Boyle County. Oil shale \#4, quarry on N. side of road, western edge of Junction City (3 sl.).

Clark County. Oil shale \#5, old quarry $1 / 2$ mi E. of Indian Fields on the Winchester-Clay road (1 sl.).

Elliott County. Oil shale \#13, head of Corn Hollow on the Charles LeMaster farm at the headwaters of Big Sinking Creek (4 sl.).

Jefferson County. Oil shale \#9 material from well digging about $1 \mathrm{mi} \mathrm{S}$. of Twin Oaks Park on the Ash Bottom road (1 sl.).

Lewis County. Oil shale \#3 roadcut sample about $1 \frac{1 / 2}{2} \mathrm{mi}$ from Vanceburg on the Maysville road ( $1 \mathrm{sl}$.).

Lincoln County. Oil shale \#10 roadcut sample about $1 \mathrm{mi}$ from Milidgeville on the Black Pike (1 sl.).

Marion County. Oil shale \#11, quarry in Ryder's cemetery about $1 / 2 \mathrm{mi} \mathrm{E}$. of Lebanon on the Lebanon-Danville Pike (1 sl.).

Morgan County. Black shale, labeled "cannel slate" from W. R. Jillson, 12/13/20, from 2-ft outcrop in creek on the farm of Dr. J. Gullett, $1 / 2 \mathrm{mi}$ above Wrigley ( $2 \mathrm{sl}$.).

Powell County. About $1 \mathrm{mi}$ E. of Clay City (7 sl.).

Rockcastle County. Oil shale \#1, roadcut on Boone Highway at Gum Sulphur, S. side Gum Sulphur Creek (2 s1.).

Osr 4; 13 sl. Rowan County. Oil shale \#8, top of the deposit just below the soapstone quarry of the Rowan County Freestone Co. (2 sl.).

Taylor County. Oil shale \#14, outcrop in Robinson's Creek near Mansville (4 sl.).

Webster County. Oil shale \#2, Pottsville Formation (from W. R. Jillson, Sept. 1920, Harden Asher farm near Tilden).

Cannel (slate?) Upper Pottsville, horizontal (1 sl.).

Kentucky shale (2 sl.). 
Oil Shale and Related Rocks, U.S.-Continued

Osr 5; 13 sl. Montana.

Oil shale, Medicine Lodge Basin, $10 \mathrm{mi} \mathrm{SW}$. of Armstead, Lemhi County ( 3 sl. hard shale +1 block).

New York.

Dunkirk black shale. Devonian (1 sl.).

Geneseo Shale. Taughannock Falls Park, Tompkins County, W. side of Lake Cayuga. (From J. M. S., 9/7/52. 6 sl.)

Geneseo Shale. Lively Run near Sheldrake, W. side of Lake Cayuga, Seneca County. (From J. M. S., 9/7/52. 1 sl.)

Skaneateles marine shale. Concretions, Fayette quarry, near Fayette, Seneca County ( 2 sl.).

Osr 6; 13 sl. Oklahoma.

Carbonaceous sandstone. Nowata Oil Field, Nowata County. Specimens (core samples) transmitted to Coal Geology Laboratory Feb. 1956. (Includes 4 polished blocks.)

Osr 7; 19 sl. Colorado.

Oil Shale. Rifle, Garfield County ( 3 sl. oil shale, 1 sl. asphaltic sandstone below the shale).

Utah.

Oil shale. Wales, Sanpete County, "Wales" bed, North Horn Formation (2 sl.).

Wyoming.

Oil shale. Fossil, Lincoln County, Green River Formation (13 sl.).

\section{FOREIGN}

Osr 8; 8 sl. Australia. Neunes, Sydney, New South Wales Kerosene shale from John Fell ( 8 sl.).

Osr 9; 4 sl. Canada.

Nova Scotia. Torbanite (bedded oil shale). Stellarton, Pictou County. (From A. E. Flynn. 2 sl.)

Nova Scotia. (Black shale from J. M. S., 6/22/52, 1 sl. + fragment.)

New Brunswick. Albertite, "Albert Coal" (1 sl.).

Osr 10; 6 sl. China. (From China Geol. Survey.)

Lopingite? (high cuticle). Loping, Kiangsi Province (2 sl.).

Impure Coal? Liling, Hunan Province (1 sl.); Hünyuan, Shansi Province (2 sl.); Shun-ching, Anhwei Province (1 sl.)

Osr 11; 2 sl. $\quad$ Estonia.

Kukersite. Marine oil shale, Ordovician.

Osr 12; 2 sl. $\quad$ France.

Boghead. Margenne, Autun, Soc. Lyonaise de schiste Bitum. Harvard Museum \#.52831 (1 sl. + 1 lump).

Morocco. E.C.E. exchange sample, 1952 Mineral Hydrocarbon (1 sl.). 
Oil Shale and Related Rocks, Foreign - Continued

Osr 13; 20 sl. Peru. Asphaltic material.

Canta. Mariag, near Lima? Sample \#11 (5 sl.).

Huancayo. Yauricocha. Sample \#14 (5 sl.).

Huarochevic. Sillapata. Sample \# 1 (2 sl.).

Jauja. Santos, asphaltic. Sample \#8 (3 sl.).

Pasco. Goyllarisquizga. 3 sl. coal Sample \#12 (5 sl.).

Osr 14; 24 sl. Peru.-Continued.

Yauli. Chuicho. Sample' \#6 (12 sl.).

Harvis (or Hauris?) asphaltic coal. Sample \#5 (5 sl.).

Lassachocha. Sample \#7 (2 sl.).

Pachacayo. Sample \#7 (3 sl.).

Pomalcha. Sample \#4 (2 sl.).

Osr 15; 16 sl. Peru.-Continued.

Yauli. Sorao. Sample \#15 (5 sl.).

Yantac. Sample \# 9 (5 sl.).

+ 6 slides carbonaceous material. No locality (Numbered 4, 9, 10. Probably sample numbers.)

Osr 16; 7 sl. Scotland. Torbanite, Torbane Hill, 6/28/50.

Osr 17; 11 sl. Scotland. Scottish oil shale. Broxburn shale, Broxburn mine, 1950. Forkneu-Arib Uphall 32. (3 sl. from the mine +8 sl. from Broxburn area.)

Osr 18; 2 sl. $\quad$ Sweden. Black shale(?), KOLM, OX1AB.

Osr 19; 14 sl. Union of South Africa. Cuticle coal and oil shale from Molteno coal (1 sl.).

Union of South Africa. Satmar "B" coal, torbanite. Satmar Mining Co., about $8 \mathrm{mi}$ N. of Ermelo (8 sl.).

Union of South Africa. Umkomaas "oil shale." Natal. (From J. M. S., July 1947. 4 sl.)

Africa? "Wood" fragments. A. Dutoit. Kimberly (1 sl.).

\section{PEAT SECTIONS}

Pt 1; 16 sl. Florida. Everglades National Park, Dade County, location EE-10, $2 \mathrm{mi} \mathrm{W}$. of Rt. 27, 71/2 mi N. of West Lake (5 sl.); location EE-12, $1 / 2 \mathrm{mi}$ E. of Rt. 27, 81/2 mi N. of Long Lake (7 sl.). (From W. Spackman, 1958.)

Midway Key, Everglades National Park, Monroe County. (From Red Mangrove roots (compressed). GSA Trip \#6, Stop 14, Nov. 1974. 4 sl.)

Pt 2; 6 sl. Pennsylvania. Bridgville swamp, Allegheny County (Thiessen microtome sections of peat wood).

Pt 3; 19 sl. Wisconsin. Peat wood, carbonized peat and compressed peat. Thiessen collection (5 microtome slides, 14 thin sections).

Pt 4; 21 sl. Peat wood, Thiessen microtome sections. 


\section{REFERENCES CITED}

Allman, Michael and Lawrence, D. F., 1972, Geological laboratory techniques: New York, Ärco Publishing Co., 335 p.

Ashley, G. H., 1920, A use classification of coal: Am. Inst. Mining Engineers Trans., v. 63, p. 782-796; Am. Inst. Mining Engineers Bull. 152, p. 1129; Coal Age, v. 16, no. 23, p. 918-925.

1923, A practical classification of coals: Coal Mining Inst. America, 37th Ann. Mtg. (Pittsburgh), Proc., p. 29-40.

1926, A practical classification of coals: Pennsylvania Geol. Survey, 4th Ser., Bull. 89, 16 p. (mimeo.)

Jeffrey, E. C., 1916, Methods of studying coal: Sci. Conspectus, v. 6, p. 71-76.

Jeffrey, E. C., and Chrysler, M. A., 1906, The lignites of Brandon: Vermont State Geologist, 5th Rept., p. 195-201.

Lomax, James, 1911, The microscopical examination of coal: Inst. Mining Eng. Trans., v. 42, p. 2-15.

1922, The preparation of transparent sections of coal: Fuel in Science and Practice, v. 1, p. 79-84.

Parks, B. C. and O'Donnelly. H. J., 1949, Determination of petrographic components of coal by examination of thin sections (with discussion): Am. Inst. Mining Engineers, Trans. [Coal Div. 1948], v. 177, p. 535-555.

1956, Petrography of American coals: U.S. Bur. Mines Bull. 550, 193 p.

Schopf, J. M., 1956, Petrologic methods for application of solid fuels of the future: Am. Inst. Mining Engineers Trans., v. 205, p. 629-639; Mining Eng., June 1956, $11 \mathrm{p}$.

Sprunk, G. C., 1942, Influence of physical constitution of coal upon its chemical hydrogenation and carbonation properties: Jour. Geology, v. 50, p. 411-436.

Stopes, M. C., 1919, On the four visible ingredients in banded bituminous coal: Royal Soc. London Proc., Ser. B, v. 90, p. 470-487.

Stopes, M. C., and Wheeler, R. V., 1918, Monograph on the constitution of coal: London, Dept. Sci. Indus. Research, 58 p.

\section{BIBLIOGRAPHY OF REINHARDT THIESSEN}

1908

The vascular anatomy of the seedling of Dioon edule: Bot. Gazette, v. 46, p. 357-380, 7 pls.

1911 1912

Plant remains composing coals [abs.]: Science, new ser., v. 33, p. 551-552.

On certain constituents and the genesis of coals [abs.]: Washington Acad. Sci. Jour., v. 2, p. 232-233.

1913

On the constitution and genesis of certain lignites and sub-bituminous coals [abs.]: 8th Internat. Cong. Applied Chemistry, Original Communications, v. 25 (1912), p. 203-204. 1918

The origin of coal (with White, David): U.S. Bur. Mines Bull. 38, 390 p.

The determination of the stratigraphic position of coal seams by means of their 1919

spore-exines [abs.]: Science, new ser., v. 47, p. 469.

Constitution of coal through a microscope: Coal Min. Inst. America, 33rd Proc., p. 34-44, discussion, p. 44-45; Coal Industry, v. 2, p. 558-563. 
1919

Occurrence and origin of finely disseminated sulphur compounds in coal: Am. Inst. Mining Engineers, B 153, 1919, p. 2431-2444; Trans., v. 63, 1919-20, p. 913-926; Trans., v. 63, 1919-20, p. 926-931; Coal Age, v. 16, 1919, p. 668-673. 1920

Structure in Paleozoic bituminous coals: U.S. Bur. Mines Bull. 117, 296 p.

Structure in bituminous coals: U.S. Bur. Mines Rept. Inv. 2196, 4 p.

The correlation of coal seams by means of spore exines [abs.]: Science, new ser., v. 51, p. 522 .

Compilation and composition of bituminous coals: Jour. Geology, v. 28, p. 185-209.

Recent developments in the microscopic study of coal: Coal Min. Inst. America, 1920-21

34th Proc., 1920, p. 88-119; discussion, p. 119-120.

Under the microscope coal has already lost much of its former mystery: Coal Age,

v. 18, 1920, p. 1183-1189, 1223-1228, 1275-1279; v. 19, 1921, p. 12-15. 1921

Origin and composition of certain oil shales: Econ. Geology, v. 16, no. 4-5, p. 289-300, 2 pls. [abs.] discussion by F. R. Van Horn, Geol. Soc. America Bull., v. 32 , no. 1, p. $72-74$.

1922

A microscopic study of the Freeport Coal Bed, Pennsylvania (and Voorhees, A. W.): 1923

Carnegie Inst. Technology Bull. 2, 75 p.

Correlation of coal beds in the Monongahela Formation of Ohio, Pennsylvania, 1924

and West Virginia (and Staud, J. N.): Carnegie Inst. Technology Bull. 9, 64 p.

The origin and constitution of coal: Wyoming [Pa.] Hist. and Geol. Soc., Proc. and Coll., v. 19, 44 p.

Correlation of coal beds of the Allegheny Formation of Western Pennsylvania and Eastern Ohio (and Wilson, R. E.): Carnegie Inst. Technology Bull. 10, 56 p. 1925

The microscopical constitution of coal: Am. Inst. Mining Engineers Pamphlet 1438, 50 p.; Trans., v. 71, p. 35-114; Pamphlet 1473, discussion p. 12-14; Trans., v. 71, p. 114-116.

Microscopic examination of Kentucky oil shales: Kentucky Geol. Survey, Ser. 6, v. 21 , p. $1-47,37$ pls.

Origin of the boghead coals. Shorter contributions to general geology, 1923-24: U.S. 1926

Geol. Survey Prof. Paper 132-I, p. 121-137.

The microstructure of coal: Royal Soc. Arts (London), Jour., v. 74, p. 535-554; dis1928 cussion, p. 554-557.

Some recent developments on the constitution of coal: 2nd Internat. Conf. Bitumi1929

nous Coal (Pittsburgh), Proc., v. 1, p. 695-761; discussion, p. 761-767.

The microscopic structure of coals of the Monongahela Series: West Virginia Acad.

Sci., Proc., v. 3, p. 159-198. 
Terminology in coal research (and Francis, W.): U.S. Bur. Mines Tech. Paper 446, 27 p.; Fuel, v. 8, p. 385-406. 1928-30

Classification of coal from the viewpoint of the paleobotanist: Am. Inst. Mining Engineers Tech. Pub. 156, 1928, p. 28-44; discussion, p. 44-46; Coal Div., Trans., v. 88, 1930, p. 419-435; discussion, p. 435-437. 1929-30

An analysis of a peat profile (and Johnson, R. C.): Industrial Engineering Chemistry, 1930

Analytical Edition, v. 1, 1929, p. 216-220; Fuel, v. 9, 1930, p. 367-372.

Splint coal: Am. Inst. Mining Engineers Trans., v. 88, p. 644=671, discussion, p. 671-672.

1930-31

Recently developed methods of research in the constitution of coal and their application to Illinois coals. Illinois State Geol. Survey, Coop Mining Series Bull. 33, 1930, p. 58-59; Bull. 60, 1931, p. 117-147; Fuel, v. 10, 1931, p. 72-94. 1931

Methods and apparatus used in determining the gas-, coke-, and byproduct-making properties of American coals, with results on a Taggart-Bed Coal from Rhoda, Wise County, Va. (with Fieldner, A. C., Davis, J. D., and Kester, E. B., Selvig, W. A.): U.S. Bur. Mines Bull. 344, 107 p., 53 figs.

Microscopic study of Elkhorn Coal Bed at Jenkins, Letcher County, Ky. (with ,Sprunk, 1932

G. C., and O'Donnell, H. J.): U.S. Bur. Mines Tech. Paper 506, 30 p.

Physical structure of coal, cellulose fiber, and wood as shown by the Spierer Lens: Industrial \& Engineering Chem., v. 24, p. 1032-1041.

Carbonizing properties and constitution of Chilton Bed Coal from Boone No. 2 Mine, Logan County, W. Va. (with Fieldner, A. C., Davis, J. D., and Kester, E. B., Selvig, W. A., Reynolds, D. A., Jung, F. W., Sprunk, G. C.): U.S. Bur. Mines Tech. Paper 542, $60 \mathrm{p}$.

Carbonizing properties and constitution of Black Creek Bed Coal from Empire Mine, Walker County, Ala.: U.S. Bur. Mines Tech. Paper 531, 44 p.

Carbonizing properties and constitution of Pittsburgh Bed Coal from Edenborn Mine, Fayette County, Pa. (with Fieldner, A. C., Davis, J. D., and Kester, E. B., Selvig, W. A., Reynolds, D. A., Jung, F. W., Sprunk, G. C.): U.S. Bur. Mines Tech. Paper 525, $60 \mathrm{p}$.

Carbonizing properties and constitution of No. 6 Bed Coal from West Frankfort, Franklin County, Ill.: U.S. Bur. Mines Tech. Paper 524, 60 p.

Carbonizing properties and constitution of washed and unwashed coal from Mary Lee Bed, Flat Top, Jefferson County, Ala.: U.S. Bur. Mines Tech. Paper 519, 78 p.

Spores of certain American coals (and Sprunk, G. C.): Fuel, v. 11, p. 360-370.

Carbonizing properties and constitution of No. 2 Gas Bed Coal from Point Lick No. 4 Mine, Kanawha County, W. Va. (with Fieldner, A. C., Davis, J. D., and Kester, E. B., Selvig, W. A., Reynolds, D. A., Jung, F. W., Sprunk, G. C.): U.S. Bur. Mines Tech. Paper 548, 52 p. 
Studies on peat: Alcohol and ether-soluble matter of certain peats (and Johnson, R. C.): Fuel, v. 13, p. 44-47.

The effect of heat on coal as revealed by the microscope (and Sprunk, G. C.): Fuel, v. 13, p. 116-125.

The isolation and study of the humic acids from peat (with Arnold, C. L., Lowy, Alexander): U.S. Bur. Mines Rept. Inv. 1934, 9 p.; Fuel, v. 14, 1935, p. 107-112.

The distribution of microorganisms in four peat deposits (and Strickler, H. S.): Carnegie Inst. Tech. Coop. Bull. Mining and Metall. Inv., 61, 19 p.

Relation of microscopic composition of coal to chemical, coking and by-product properties (and Sprunk, G. C.): Industrial \& Engineering Chem., v. 27, p. 446-451.

Microscopic and petrographic studies of certain American coals: U.S. Bur. Mines Tech. Paper 564, 71 p.

Carbonizing properties and constitution of Alma Bed Coal from Spruce River No. 4 Mine, Boone County, W. Va. (with Fieldner, A. C., Davis, J. D., and Kester, E. B., Selvig, W. A., Reynolds, D. A., Jung, F. W., Sprunk, G. C.): U.S. Bur. Mines Tech. Paper 562, 41 p.

1936

Carbonizing properties and petrographic composition of Clintwood-Bed Coal from Buchanan Mines Nos. 1 and 2, Buchanan County, Va.: U.S. Bur. Mines Tech. Paper $570,34 \mathrm{p}$.

Carbonizing properties and petrographic composition of Pittsburgh Bed Coal from Pittsburgh Terminal No. 9 Mine, Washington County, Pa.: U.S. Bur. Mines Tech. Paper 571, 33 p.

Origin of the finely divided or granular opaque matter in splint coals (and Sprunk, G. C.): Fuel in Science and Practice, v. 15, p. 304-315.

1936-37

What is Coal?: Fuel engineer's meetings sponsored by Fuel Eng. Div., Appalachian

Coal Inc., Record of Meetings, v. 4, p. 211-272; discussion, p. 273-279. 1937

Origin and petrographic composition of the Lower Sunnyside Coal of Utah (and Sprunk, G. C.): U.S. Bur. Mines Tech. Paper 573, 34 p.

Carbonizing properties and petrographic composition of Millers Creek Bed Coal from Consolidation No. 155 Mine, Johnson County, Ky., and the effect of blending Millers Creek Coal with Pocahontas-Bed and Pittsburgh-Bed (Warden Mine) Coals (with Fieldner, A. C., Davis, J. D., and Selvig, W. A., Reynolds, D. A., Sprunk, G. C., Holmes, C. R.): U.S. Bur. Mines Tech. Paper 572, 50 p.

1938

Carbonizing properties of West Virginia coals and blends of coals from the Alma, Cedar Grove, Dorothy, Powellton A, Eagle, Pocahontas, and Beckley Beds (with Fieldner, A. C., Davis, J. D., Selvig, W. A., and Reynolds, D. A., Holmes, C. R., Sprunk, G. C.): U.S. Bur. Mines Bull. 411,162 p. 
Carbonizing properties and petrographic composition of Upper Banner-Bed Coal from Clinchfield No. 9 Mine, Dickenson County, Va., and of Indianan No. 4-Bed Coal from Saxton No. 1 Mine, Vigo County, Ind., and the effect of blending these coals with Beckley Bed Coal (with Fieldner, A. C., Davis, J. D., and Selvig, W. A., Reynolds, D. A., Brewer, R. E., Sprunk, G. C.): U.S. Bur. Mines Tech. Paper 584, $81 \mathrm{p}$.

Preparation of thin sections of coal (with Sprunk, G. C., and O'Donnell, H. J.): U.S. Bur. Mines Inf. Circ. 7021, 8 p.; Fuel, v. 17, p. 307-315. 1939

Carbonizing properties and petrographic composition of washed and unwashed Lowe and Upper Kittanning-Bed Coals from Mines 72 and 73, Johnstown, Cambria County, Pa. (with Fieldner, A. C., Davis, J. D., and Selvig, W. A., Reynolds, D. A., Holmes, C. R.): U.S. Bur. Mines Tech. Paper 595, 83 p. 1941 1947

Coal paleobotany: U.S. Bur. Mines Tech. Paper 631, 56 p.

What is coal?: U.S. Bur. Mines Inf. Girc. 7397, 53 p.; reprinted from Appalachian Coal Fuel Engrs. Conf., Proc., v. 4, 1936-37, p. 211-279. 




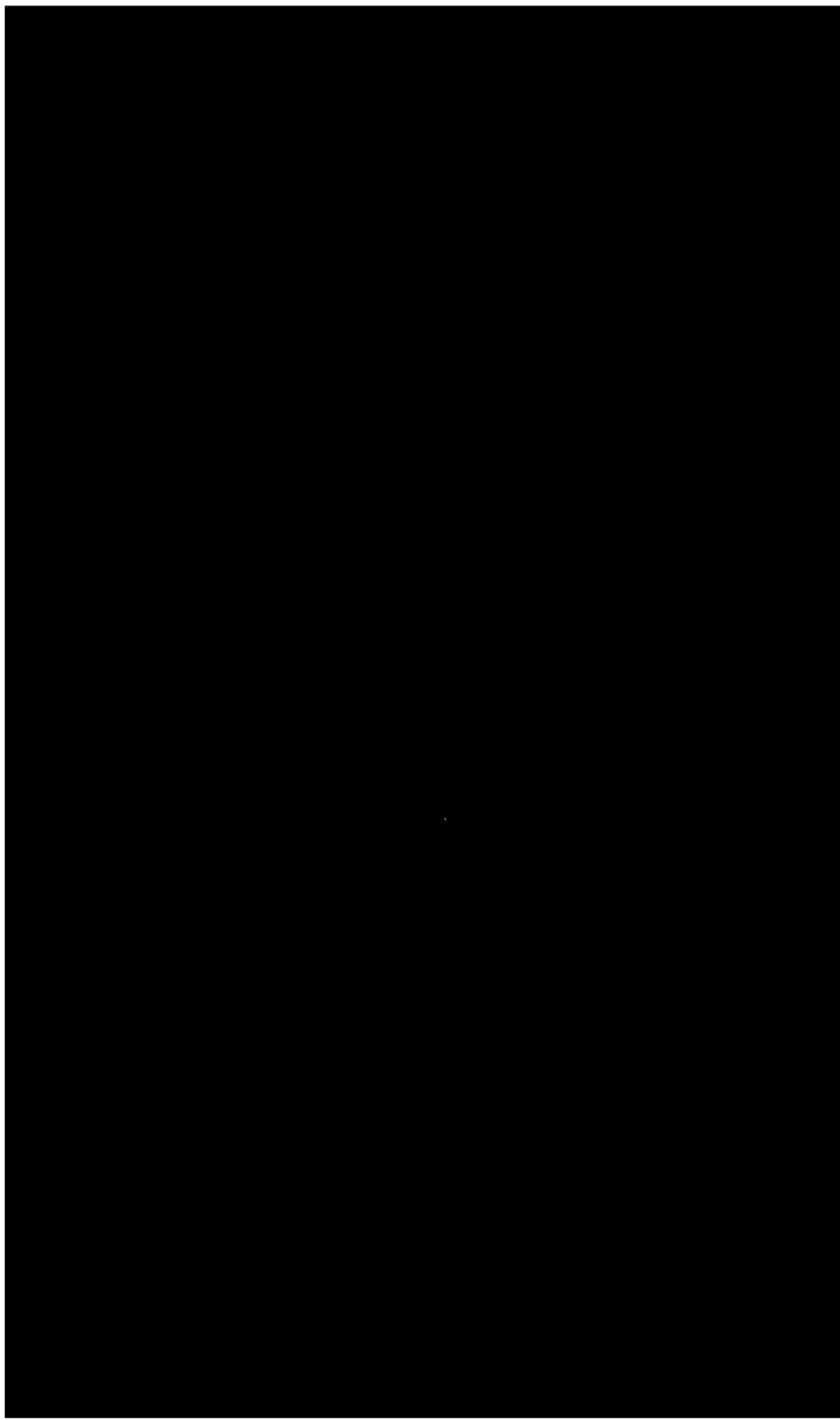




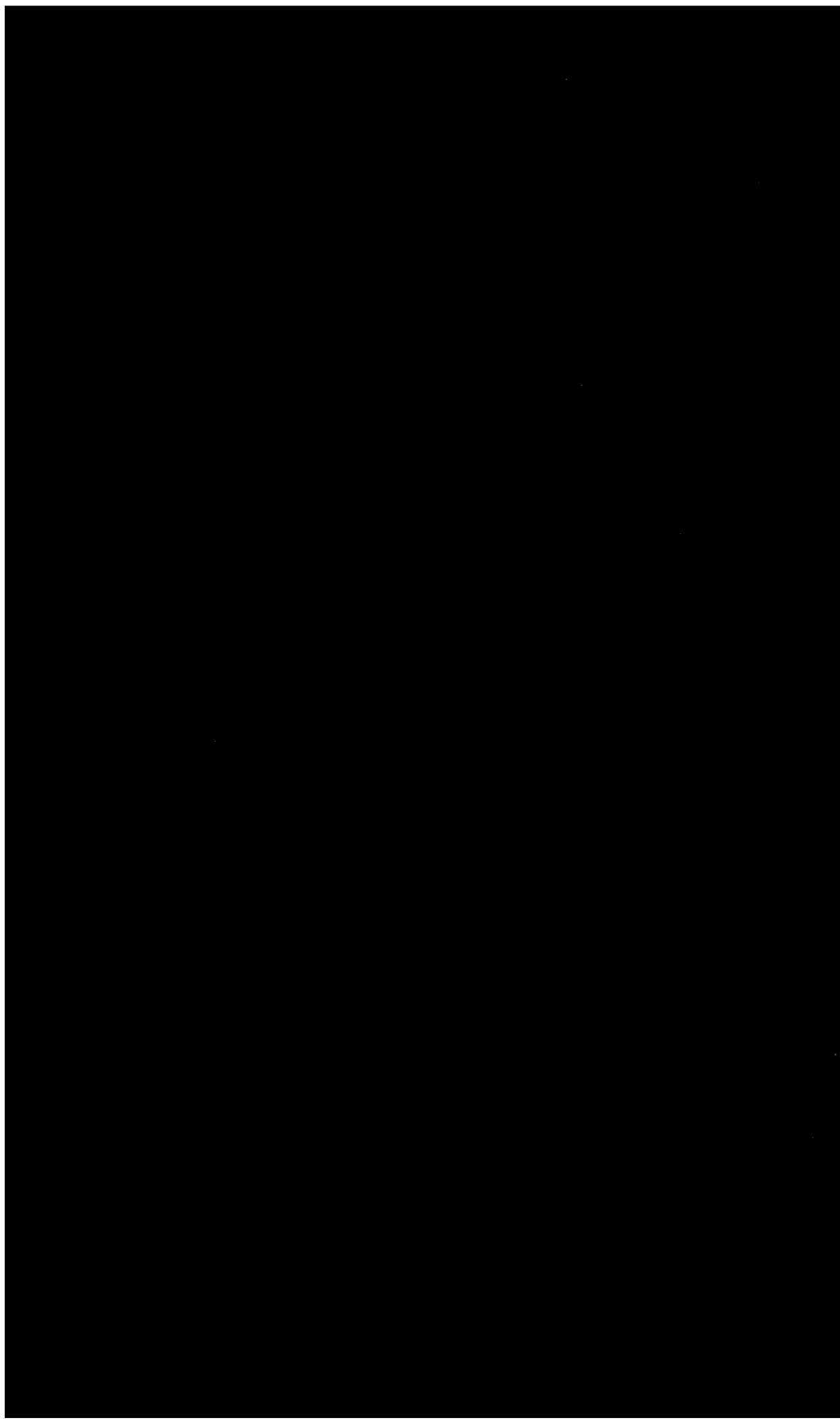

